\title{
THE ANNUAL MEETING IN ROCHESTER
}

The sixty-third Annual Meeting of the American Mathematical Society was held at the University of Rochester on Thursday through Saturday, December 27-29, 1956, in conjunction with meetings of the Mathematical Association of America on Saturday, December 29 and the Association for Symbolic Logic on Thursday, December 27. The registration was 615 including 508 members of the Society.

The thirtieth Josiah Willard Gibbs Lecture, entitled Mathematics and the future of science, was delivered by Professor M. H. Stone of the University of Chicago on Thursday evening. Professor Richard Brauer, President Elect of the Society, presided.

By invitation of the Committee to Select Hour Speakers for Annual and Summer Meetings, Professor D. C. Spencer of Princeton University delivered an address, entitled On complex structures, on Thursday afternoon at a session presided over by Professor A. C. Schaeffer.

On Friday morning the Frank Nelson Cole Prize in Number Theory was awarded to Professor J. T. Tate of Harvard University for his paper The higher dimensional cohomology groups of class field theory which appeared in volume 56 of the Annals of Mathematics. Professor Tate addressed the Society briefly on the subject of his prize-winning memoir.

Tea was served on Thursday afternoon in the Faculty Club, and there was a beer party in the Men's Dining Hall after the Gibbs Lecture on Thursday evening. A conducted tour of the George Eastman House Museum of Photographic Art was held on Friday afternoon.

A banquet was held in the dining room of the Women's Residence Hall on Friday evening. Professor J. F. Randolph of the University of Rochester acted as toastmaster. The speakers were Mr. Sol Linowitz, a trustee of the University of Rochester, Professor Richard Brauer, President Elect of the American Mathematical Society, Dean W. L. Duren, President of the Mathematical Association of America, Professor S. C. Kleene, President of the Association for Symbolic Logic, and Professor M. H. Stone. A resolution of thanks to the University of Rochester was presented by Professor R. L. Jeffery.

The Annual Business Meeting of the Society was held on Friday, December 28, 1956. The Secretary reported that at this time the ordinary membership of the Society is now 5271 , including 572 nominees of institutional members and 31 life members. There are also 
136 institutional members. The total attendance of members at all meetings in 1956 was 2376 ; the number of papers read was 884 ; there were 14 hour addresses; 1 Gibbs Lecture and 9 papers at an Applied Mathematics Symposium. The number of members attending at least one meeting was 1520.

Professor J. F. Randolph reported for the tellers that a total of over 1300 ballots had been cast in the election and the following were elected:

Vice President, Professor Salomon Bochner.

Treasurer, Dean A. E. Meder, Jr.

Secretary, Professor J. W. Green.

Associate Secretary, Professor R. D. Schafer.

Member of the Editorial Committee of the Bulletin, Professor B. J. Pettis.

Member of the Editorial Committee of the Proceedings, Professor Irving Kaplansky.

Member of the Editorial Committee of the Transactions and Memoirs, Professor S. S. Chern.

Member of the Editorial Committee of the Colloquium Publications, Professor E. J. McShane.

Member of the Editorial Committee of Mathematical Reviews, Professor R. P. Boas, Jr.

Member of the Editorial Committee of Mathematical Surveys, Dr. S. M. Ulam.

Member of the Committee on Printing and Publishing, Professor E. G. Begle.

Representative on the Board of Editors of the American Journal of Mathematics, Professor Harish-Chandra.

Members of the Board of Trustees, Professor Deane Montgomery, Dean Mina Rees, and Professor G. A. Hedlund.

Members-at-large of the Council, Dr. R. E. Bellman, and Professors Lipman Bers, A. M. Gleason, P. R. Halmos and D. H. Lehmer.

The Council met on Thursday afternoon, December 27, 1956.

The Secretary announced the election of the following fifty-two persons to ordinary membership in the Society:

Miss Esther Adler, Columbia University;

Professor O. R. E. Barker, Hampton Institute;

Mr. George Biriuk, Naval Avionics Facility;

Mr. R. E. Bolger, Fairfield University;

Mr. R. E. Briney, Massachusetts Institute of Technology;

Dr. Johanna H. M. Brunings, Ramo-Wooldridge Corporation;

Sister M. L. A. Colbert, Marylhurst College; 
Professor R. B. Crouch, New Mexico College of Agriculture and Mechanic Arts;

Dr. D. A. Edwards, Yale University;

Mr. Herbert C. Field, Purdue University;

Dr. Seymour Haber, Weizmann Institute of Science;

Miss Susan G. Hahn, Institute of Mathematical Sciences, New York University;

Mr. Milton Halem, New York University;

Dr. Haim Hanani, Israel Institute of Technology;

Mr. D. M. Hess, Fordham University;

Professor C. V. Holmes, San Diego State College;

Reverend F. A. Homann, S. J., University of Pennsylvania;

Mr. R. H. Homer, University of California, Berkeley;

Mr. T. J. Horrigan, Cook Research Laboratories, Skokie, Illinois;

Mr. J. E. Houle, Georgetown University;

Mr. L. A. Johnson, Taylor Pipe and Forge Works;

Mr. J. J. Kaganove, Armour Research Foundation;

Professor C. E. Kerr, Lafayette College;

Dr. Lester Kraus, Convair, San Diego, California;

Mr. P. V. LaGrange, Jr., The RAND Corporation;

Mr. Eric Liberman, Elite Furniture Company, Bulawayo, S. Rhodesia;

Mr. W. E. Loper, U. S. Naval Ordnance Test Station;

Reverend Anthony Mardellis, Long Beach State College;

Dr. R. L. Marti del Castillo, Banco Nacional de Cuba, Habana, Cuba;

Mr. Jack Minker, RCA Victor, Camden, New Jersey;

Professor T. F. Mulcrone, S. J., Loyola University;

Mr. P. D. Oyer, National Security Agency;

Mr. P. C. Patton, Harvard University;

Miss Nelly E. Reitlinger, University of Michigan;

Dr. Nicholas Rescher, RAND Corporation, Santa Monica, California;

Professor B. E. Rhoades, Lafayette College;

Dr. W. J. Riordan, Bell Telephone Laboratories, Whippany, New Jersey;

Dr. R. C. Roberts, Naval Ordnance Laboratory, Silver Spring, Maryland;

Miss Grace A. Sacks, International Business Machines, New York, New Yørk;

Mr. P. J. Sally, Jr., Parke Mathematical Laboratories, Carlisle, Massachusetts;

Professor Diran Sarafyan, Lamar State College of Technology;

Dr. Berthold Schweizer, Illinois Institute of Technology;

Dr. R. G. Segers, Bell Telephone Laboratories;

Professor D. R. Sudborough, Central Michigan College;

Mr. G. M. Taylor, North American Aviation, Los Angeles, California;

Mr. F. A. Varrichio, St. Peter's College;

Dr. H. M. Wachowski, The Ramo-Wooldridge Corporation;

Professor E. E. Walden, New Mexico College of Agriculture and Mechanic Arts;

Dr. A. D. Wasel, Lockheed Aircraft Corporation;

T/Sgt. R. E. Wheeler, U. S. Air Force, Seattle, Washington;

Dr. Morris Yachter, The M. W. Kellogg Company, New York, New York.

It was reported that the following one hundred and ninety persons had been elected to membership on nomination of institutional members as indicated:

Alabama Polytechnic Institute: Professor D. E. Johnson.

University of Alabama: Professor R. N. Festa. 
University of British Columbia: Mr. Bomshik Chang, Mr. Jih-ou Chow, and Mr. I. H. Mufti.

Brooklyn College: Mr. C. F. Ricciardelli.

Brown University: Dr. A. G. Azpeitia, Professor Maurice Holt, and Professor L. M. Milne-Thomson.

California Institute of Technology: Mr. George Gerson, Mr. Morton Lowengrub, Mr. J. R. Rice, and Mr. P. M. Weichsel.

University of California, Berkeley: Mr. R. O. Abernathy, Mr. H. S. Bear, Mr. Lensey Chao, Mr. R. P. Holten, Mr. H. H. Johnson, Miss Eva M. Kallin, Mr. Milton Lees, Mr. A. W. McKinney, III, Mr. J. D. Monk, Mr. J. E. Ohm, Mr. M. R. Porter Mr. J. A. Simmons, Mr. S. F. Tuan, and Mr. C. E. Watts.

University of California, Los Angeles: Mr. A. M. Bruckner, Mr. Seymour Goldberg, Mr. K. M. Larsen, Mr. P. A. Nickel, and Mr. J. F. Seewerker.

Case Institute of Technology: Mr. W. O. Portmann.

The University of Chicago: Mr. J. D. Halpern, Mr. M. W. Hirsch, Mr. R. C. O'Neil, Mr. J. J. Rotman, Mr. J. G. Thompson, Mr. Jacob Towber, and Mr. J. A. Wolf.

Columbia University: Mr. Arthur Babakhanian, Mr. E. W. Chamberlain, Mr. N. P. Herzberg, Mr. J. D. Lubin, Mr. Robert Morixe, Mr. W. V. Petryshyn, Mr. S. D. Ross, and Mr. Jerrold Rubin.

Cornell University: Dr. Harry Kesten, Mr. D. B. Lissner, Dr. Wolfgang Rindler, Mr. J. D. Rutledge, and Professor Ambikeshwar Sharma.

Duke University: Dr. A. M. Chak, Mr. Auguste Forge, Mr. G. E. Goode, Mr.

A. S. Joyner, Mr. W. L. Messmer, and Mr. E. H. Moore, Jr.

University of Florida: Mr. J. B. Wilson.

Harvard University: Mr. Michael Artin, Mr. J. A. Cohn, Mr. P. L. Falb, Mr. Paul

Fong, Mr. M. H. Greenberger, Mr. M. E. Harris, Mr. E. S. Loebenstein, Mr. D. M.

Mandelbaum, Mr. Albert Marden, Mr. A. P. Ogg, and Mr. V. C. Williams.

University of Illinois: Mr. D. W. Dean, Mr. C. C. Farrington, Jr., Mr. J. L.

Goldberg, Mr. M. I. Knopp, Mr. E. A. Newburg, Miss Rosemarie S. Stemmler, Mr.

L. T. Wos, and Mr. Peter Yff.

Indiana University: Mr. R. P. Kanwal.

Institute for Advanced Study: Dr. A. E. Dold, Dr. H. B. Griffiths, Dr. K. W.

Gruenberg, Dr. W. P. A. Klingenberg, Dr. H. W. Leopoldt, Dr. F. M. Ragab, Dr.

Frank Rhodes, and Dr. Sadayuki Yamamuro.

Iowa State College of Agriculture and Mechanic Arts: Mr. R. D. Low.

State University of Iowa: Mr. G. S. Rogers.

University of Kansas: Mr. C. R. Deeter, and Mr. S. A. Khabbaz.

Kenyon College: Mr. T. M. Jenkins.

Lehigh University: Miss Hanna I. Nassar, and Mr. R. C. Scott.

University of Maryland: Mr. H. C. Berry, Mr. D. W. Fox, Professor W. H. Greub,

Dr. H. R. A. Holmann, Mr. P. H. Maserick, Mr. R. E. McGill, and Mr. G. H. Weiss. Massachusetts Institute of Technology: Mr. R. R. O'Brien, Dr. K. F. Roth, Mr.

J. R. Schue, and Dr. J. T. Stuart.

University of Michigan: Mr. J. P. Benkard, Mr. G. T. Cargo, Mr. C.-Y. Chao, Mr. P. T. Church, Mr. Oswald Petrucco, Mr. Arnold Steiken, Mr. E. S. Simons, and Mr. W. G. Weideman.

University of Minnesota: Mr. S. D. Burgstahler, Mr. R. D. Driver, Mr. J. A. Lindberg, Jr., Mr. D. E. Varberg, and Mr. J. J. Yeh.

University of Nebraska: Mr. L. D. Fountain. 
University of New Hampshire: Mr. F. J. Lorenzen, Jr.

Northwestern University: Miss Ethel J. Allard, Mr. D. M. Danvers, and Mr.

K. E. Hillstrom.

Oklahoma Agricultural and Mechanical College: Mr. T. K. Boehme, Mr. T. W.

Cairns, and Miss Leone Yarborough.

University of Oregon: Mr. J. R. Borsting.

Pennsylvania State University: Mr. R. T. Heimer.

University of Pennsylvania: Mrs. Bonnie Averbach, Mr. S. J. Einhorn, Mr. B. I.

Gross, Mr. D. J. Ostroff, and Mr. M. W. Pownall.

Purdue University: Mr. D. L. Phillips, and Mr. J. H. Stapleton.

Queens College: Dr. C. R. Chester.

Rutgers University: Mr. W. E. Gould, Mr. W. R. Jones, Mr. D. R. King, Mr.

R. A. McHaffey, and Mr. John Weissman.

College of St. Thomas: Professor R. J. Dowling.

University of Southern California: Mr. J. W. Capps, Professor H. O. Cordes, Mr. E. J. Eckert, Mr. Isidore Eisenberger, Mr. Humberto Gutierrez, and Mr. K. F. Wilson.

University of Tennessee: Mr. P. H. Doyle.

University of Texas: Mr. J. L. Cornette, Mr. J. W. Neuberger, Mr. J. H. Nicholson, and Mr. Dave Pandres, Jr.

University of Toronto: Mr. D. F. Clapp, Miss Diane M. Johnson, Mr. J. H. Lindsay, Jr., Mr. E. S. Lowry, Mr. C. A. Pegis, and Mr. F. A. Sherk.

University of Virginia: Mr. S. H. Coleman, Mr. W. D. Googe, Mr. E. H. Greene, Mr. S. H. Lawrence, and Mr. C. N. Lee.

State College of Washington: Professor Hans Schneider.

University of Washington: Mr. C. W. Clark, Mr. E. T. Kobayashi, Mr. R. D. Mayer, Mr. R. B. Paine, and Mr. K. R. Stromberg.

Wayne State University: Sister Mary Catharina Bereiter, and Mr. Louis Sucheston.

University of Wisconsin: Mr. H. F. Bechtell, Jr., Mr. W. G. Collar, Mr. R. C. Courter, Mr. G. W. Hedstrom, Mr. J. M. Kister, Mr. H. J. Rebassoo, Mr. D. A. Robinson, and Mr. R. H. Rosen.

Yale University: Mr. R. L. Adler, Mr. R. T. Barnes, Miss Eleanor Killam, Mr. P. E. Miles, Mr. J. M. Osborn, Jr., Mr. M. J. Poliferno, Mr. S. L. Salas, Miss T. R. Spiselman, and Mr. Donald Wehn.

The Secretary announced that the following had been admitted to the Society in accordance with reciprocity agreements with various mathematical organizations: Deutsche Mathematiker-Verinigung: Dr. E.-A. Behrens, Mathematicsches Seminar, Dr. G. Mayer-Kalkschmidt, University of Malaya, and Professor C. E. Mueller, Technical University; Société Mathématique de France: Professor Manuel Balanzat, Instituto de Fisica, and Dr. Paul Dedecker, Université de Liège; Svenska Mathematikersamfundet: Dr. E. H. Bareiss, David W. Taylor Model Basin, and Dr. F. A. Ryde, State Secondary School of Eksjoe; Wiskindig Genootschap te Amsterdam: Mr. P. H. Krijgsman, and Professor J. de Groot, Netherlands Postal and Telecommunications Services, The Hague, Netherlands. 
The following appointments by the President were reported: as a committee to look into the relations between the Transactions and Proceedings: J. L. Doob, Chairman, R. P. Boas, Jr., Richard Brauer, and G. T. Whyburn; as a committee to consider the establishment of annual examinations at the master's level: Leonard Gillman, Chairman, R. P. Dilworth, and D. E. Richmond; as a program committee for a Symposium on Orbit Theory to be held in April 1957: J. B. Rosser, Chairman, Garrett Birkhoff, W. Eckert, Philip Hartman, and H. Newell; as members of Committees to Select Hour Speakers (terms to expire December 31, 1958): Summer and Annual Meetings: Einar Hille (Committee now consists of J. W. Green, Chairman, G. P. Hochschild, and Einar Hille); Eastern Sectional Meetings: Warren Ambrose (Committee now consists of R. D. Schafer, Chairman, Warren Ambrose, and E. R. Kolchin); Western Sectional Meetings: A. C. Schaeffer (Committee now consists of J. W. T. Youngs, Chairman, E. H. Spanier, and A. C. Schaeffer); Far Western Sectional Meetings: Charles Loewner (Committee now consists of V. L. Klee, Chairman, Arthur Erdelyi, and Charles Loewner); Southeastern Sectional Meetings: O. G. Harrold (Committee now consists of J. H. Roberts, Chairman, E. E. Floyd, and O. G. Harrold); as a member of the Committee on Visiting Lectureships for a term of three years beginning January 1, 1957: M. R. Hestenes (Committee now consists of J. M. Thomas, Chairman, Samuel Eilenberg, and M. R. Hestenes); as the Society's representative on the U. S. National Committee for Theoretical and Applied Mechanics for a term of four years beginning January 1, 1957: William Prager; as tellers for the 1956 election: David Gale, W. G. Lister, and J. F. Randolph.

The following appointments to represent the Society were reported: at the inauguration of Reverend Louis Melbourne Hirshson as President of Hobart and William Smith Colleges on October 12, 1956: Professor T. R. Hollcroft; at the inauguration of G. C. Simpson as Chancellor of Mary Washington College on October 19, 1956: Dr. Hobart C. Carter; at the exercises dedicating the Classroom-Laboratory Building and Marking Twenty Years of Progress at Northeastern University on October 24, 1956: Professor J. L. Walsh; at the convocation in honor of Dr. Frank L. Griffin at Reed College on November 4, 1956: Professor T. S. Peterson; and at the inauguration of Warmoth Thomas Gibbs as fourth President of the Agricultural and Technical College of North Carolina on November 9, 1956: Professor W. M. Whyburn.

The Secretary reported that Professor L. M. Milne-Thomson had accepted an invitation to deliver an address at the November 30- 
December 1, 1956 meeting held at the University of Kentucky; that Professor D. C. Spencer had accepted an invitation to deliver an address at the 1956 Annual Meeting of the Society in Rochester, New York; that J. T. Tate had accepted an invitation to deliver an address at the meeting to be held in February 1957 in New Haven, Connecticut; that Professors Bernard Friedman and R. V. Kadison had accepted invitations to deliver addresses at the April 1957 meeting to be held in New York City.

The Executive Director reported that he had conducted a major membership campaign during the fall and that letters of invitation to membership had been sent out to about 15,000 people.

The Executive Director reported on the reasons for the late arrival of the October NOTICES and on the steps that had been taken to make sure that there would be no repetition of this delay.

After reviewing the make-up, printing, and distribution schedule for the NOTICES, the Council requested that the present deadline for abstracts be kept, but that preliminary announcements, as complete as possible and including reservation cards, should appear in the issue of the NOTICES just before the issue containing the program of the meeting.

The Council voted to set a meeting at the State College of Washington, Pullman, Washington, on June 15, 1957.

The Council voted that the Annual Meeting for 1958 should be held some time during the last two weeks of January 1959.

The Council voted to set Council meetings April 19, 1957, in Chicago, and at the Summer and Annual Meetings.

The following resolution was adopted by the Council:

Dr. J. V. Wehausen served as Executive Editor of Mathematical Reviews from the summer of 1950 until the summer of 1956 . This was a period of rapid growth of the mathematical literature: in these six years the amount of material reviewed was considerably more than in the preceding ten and one-half years. Dr. Wehausen coped with this flood with skill, devotion, and unfailing tact and good humor. He was resourceful in finding reviewers and in extracting reviews from them, and in extracting journals from the often (surprisingly enough) uncooperative people who issue journals. A considerable part of his success was due to the devotion which he inspired in his office staff. As the amount of material increased, he worked longer and longer hours, until at the end he was doing much more than anyone could properly have demanded. It is a tribute to his energy and resourcefulness that Mathematical Reviews today enjoys a reputation as the most complete and most informative of the 
mathematical abstracting services. It is fitting that Dr. Wehausen's services to Mathematical Reviews should be formally acknowledged. The Council, therefore, on behalf of mathematicians everywhere, extends its hearty thanks to Dr. Wehausen for his work on Mathematical Reviews.

The Council voted to elect Professor Hans Samelson to the Editorial Committee for the Proceedings to replace Professor S. S. Chern.

The Bulletin Editorial Committee reported that 652 pages had been used in 1956. The Council voted to recommend to the Board of Trustees that the Bulletin be authorized to print 625 pages in 1957.

At the request of the Editors of the Bulletin, the Council discussed the advisability of discontinuing the Research Problems section. It was the sense of the Council that this section should be continued.

The Transactions and Memoirs Editorial Committee reported that the interval between the receipt and publication of a manuscript is now approximately one year. The Council voted to request the Trustees to authorize three volumes of 550 pages each of the Transactions in 1957.

The Editorial Committee for the Proceedings reported that 1168 pages had been published in 1956 and that the Trustees had authorized the same number of pages for 1957, instead of the usual 1006 pages. The extra pages authorized by the Trustees have reduced the backlog so that the interval between receipt and publication of a manuscript is now approximately one year.

A committee consisting of J. L. Doob (Chairman), R. P. Boas, Richard Brauer, and G. T. Whyburn recommended that the Council request the Editorial Boards of the Transactions and Proceedings to interchange manuscripts as necessary in order to stabilize the Transactions publication rate at three volumes per year, with the present rough distinction between periodicals. that the Transactions prints the longer papers, serving as the guiding principle in the interchanges. The Council voted to approve this recommendation.

The Editorial Committee for Mathematical Reviews reported that the 1956 volume would be almost exactly the same size as the 1955 volume. The Committee reported finding that some members of the Society wondered whether the Council felt that Mathematical Reviews should still be considered an experiment, which could be terminated if it continues to cost too much. At the request of the Committee, the Council voted to go on record as favoring the indefinite continuation of Mathematical Reviews.

The Secretary reported that Dr. S. H. Gould had agreed to continue as Executive Editor of Mathematical Reviews.

The Joint Committee on Employment Opportunities reported that 
the number of listings in the Employment Register continues to rise.

The Committee on Translations reported that during 1956 about 900 papers had been selected for translation and were being prepared for publication. At the request of the Committee the Council voted to recommend that an increased appropriation be sought for 1957 .

There were twenty sessions for contributed papers presided over by Professor R. D. Anderson, Dr. E. H. Batho, Professors Dorothy L. Bernstein, R. H. Bruck, D. J. Dickinson, S. H. Gould, Marshall Hall, Jr., G. K. Kalisch, W. S. Loud, Mr. J. C. Mairhuber, Drs. E. W. Marchand, Steven Orey, Professors L. J. Paige, R. A. Raimi, Mary E. Rudin, Dr. Lowell Schoenfeld, Professors C. H. W. Sedgewick, M. L. Tomber, W. G. Warnock, G. W. Whitehead.

The abstracts of the papers follow. Those having the letter " $t$ " following the abstract number were read by title. Where a paper, presented in person, has more than one author, the symbol (p) follows the name of the author who presented it. Dr. Friedman was introduced by Professor Nachman Aronszajn, Professor Kallianpur by Professor Ingram Olkin, Dr. Klingenberg by Professor M. H. Heins, Professor Meyer-Koenig by Professor G. M. Merrmian, Mr. Osborn by Professor A. A. Albert, Mr. Pakshirajan by Mr. A. T. BharuchaReid, Mr. Pucci by Professor Alexander Weinstein, Dr. Rector by Professor D. M. Young, Jr., Professor Reid by Professor J. S. Frame, and Professor Sarafyan by Professor Selby Robinson.

\section{Algebra AND Theory of Numbers}

\section{Shreeram Abhyankar: Unaffected equations.}

Let $V$ be a normal $r$-dimensional algebraic variety with quotient field $K / k$ where $k$ is algebraically closed and of characteristic $p$ and let $P$ be a simple point on $V$. It is proved in [Abhyankar, On the ramification of algebraic functions, Amer. J. Math. vol. 77 (1955) pp. 575-592] that if $Q$ is a point corresponding to $P$ on a normalization of $V$ in a finite algebraic extension $L$ of $K$ and if the branch locus $D$ on $V$ for the extension $L / K$ has an $s$-fold normal crossing at $P$ then the local galois group $G(Q / P)$ is a $p_{\text {-group }}$ (definition in the above paper). Now the following construction problem is raised: Given an $(r-1)$-dimensional subvariety $D$ of $V$ having an $s$-fold normal crossing at $P$ and given a $p_{s}$-group $G$ does there exist $Q$ (in some extension $L$ of $K$ ) such that $D$ is the branch locus at $P$ and $G(Q / P$ ) is isomorphic to $G$ ? (For $r=1, G(Q / P$ ) is a $p$-group and the converse is easily settled in the affirmative, so we assume $r \neq 1$ ). The essential part of this question is the case $s=1$ and $G$ being a quasi $p$-group i.e. $G$ is generated by its $p$-sylow subgroups. Observe that for $p=2$ the symmetric group $S_{n}$ on $n$ letters is a quasi $p$-group; for this case the construction problem is settled. (Received November 13, 1956.)

148. A. A. Albert (p) and Nathan Jacobson. On the isomorphisms of the exceptional Jordan algebra.

Let $A=A(\Gamma, C)$ be the algebra of all three-rowed $J$-Hermitian matrices with elements in the Cayley algebra $C$ of dimension eight where $\Gamma$ is the diagonal matrix 
defining the involution $J$. Then we prove that two such algebras $A$ and $A_{1}=A\left(\Gamma_{1}, C_{1}\right)$ are isomorphic only if $C$ and $C_{\mathbf{1}}$ are isomorphic. (Received November 6, 1956.)

149t. A. A. Albert and L. J. Paige. Some properties of the algebras of Malcev.

Malcev has defined a Moufang-Lie algebra $M$ to be an algebra satisfying the identities (i) $x^{2}=0$; (ii) $x y \cdot x z=(x y \cdot z) x+(y z \cdot x) x+(z x \cdot x) y$. It is proved that the second identity may be replaced by the identity $w(x y \cdot z)=w y \cdot z x+(y z \cdot w) x+(w x \cdot y) z$ $+(x \cdot w z) y$. If the linear transformation $S_{x}$ is defined by the equation $a x=a S_{x}$, it is shown that trace $\left(S_{x} S_{y}\right)=F(x, y)$ is an invariant form for the algebra $M$. Moreover, the algebra $M$ is a shrinkable algebra in the sense of Albert. (Received November 13, 1956.)

\section{Rafael Artzy: Loops with identities.}

In a loop $(L,+)$ let $J$ be the mapping of each element on its right inverse so that $x+x J=0$ and $\left(x J^{k}\right) J=x J^{k+1}$, all $x \in L$, all integers $k$. Loops with the identity $(x+y) J=x J+y J$ are called automorphic-inverse. If, for some $z \in L, z J^{n}=z$, but $z J^{m} \neq z$ whenever $0<m<n$, then the $z J^{i}, i=1,2, \cdots, n$, are said to form an inversecycle of length $n$. A necessary condition for an automorphic-inverse loop to consist only of the zero and $r$ inverse-cycles of equal length $n$ is $2^{-1} r^{2} n(n+1)-r \equiv 0(\bmod n)$. This is a stronger requirement than that stated previously by the author (Proc. Amer. Math. Soc. vol. 6 (1955) p. 449). Furthermore a general method is proposed for constructing uncountably many monogenic automorphic-inverse loops of infinite order. Each of these loops satisfies one of certain additional identities, examples of which are $x+\left(x J+\left(x J^{2}+\cdots+\left(x J^{n}+y J^{n+1}\right) \cdots\right)\right)=y$, all nonzero $x, y ; n>0$; or $(z+w)+z J$ $=w$ (the crossed-inverse identity). A loop thus constructed is always the additive loop of a neofield, a generalization of Bruck's statement for the crossed-inverse case (Proc. Amer. Math. Soc. vol. 6 (1955) p. 57). (Received November 13, 1956.)

151. S. G. Bourne: On a Wedderburn-Artin structure theory of potent semirings. Preliminary report.

In a paper [S. Bourne, On multiplicative idempotents of a potent semiring, Proc. Nat. Acad. Sci. U.S.A. vol. 42 (1956) pp. 632-638] it was shown that if $S$ is a potent semiring, in which each two-sided ideal contains a minimal right ideal and a minimal left ideal of $S$, then any right ideal $R \neq(0)$ contains a multiplicative idempotent. Using the existence of this idempotent it is proved that if $S$ is a simple semiring, which contains a minimal right ideal and a minimal left ideal, then $\bar{\rho}=\left({ }_{\rho r}^{*}\right)$ where $\rho \in S, r \in R$ a minimal right ideal of $S$ and $\rho R \neq(0)$ has an inverse. Hence, if in addition $S$ possesses an identity, $S$ is isomorphic to a semiring of matrices over a division semiring. Thus, if $S$ is a potent semiring with identity and $S=\sum_{R_{i} \in \Delta} R_{i}$, where $\Delta$ is the set of all minimal right ideals $\neq(0)$ in $S$ and the decomposition is direct, then $S$ is a direct sum of semirings isomorphic to semirings of matrices over division semirings. (Received November 5, 1956.)

152. A. T. Brauer: $A$ new proof of theorems of Perron and Frobenius on positive matrices.

A well known theorem of Perron and Frobenius states that a positive matrix has a positive characteristic root $\omega$ which is greater than the absolute value of the other roots. In this paper a new proof for this result is obtained. Moreover, not only the 
existence of $\omega$ is proved, but the proof gives also a simple method to compute $\omega$ and a character istic vector belonging to $\omega$ as exactly as needed without determining the characteristic equation. The theorem of Perron and Frobenius can be formulated as follows. Every positive matrix $A$ has a greatest positive root $\omega$. This root is simple and all the other roots lie in the circle $|z|<\omega$. In this form the theorem can be improved. It will be shown that all the roots lie in the interior or on the boundary of the oval of Cassini $\left|z-a_{i i}\right|\left|z-a_{j j}\right| \leqq\left(\omega-a_{i i}\right)\left(\omega-a_{j j}\right)$ where $a_{i i}$ and $a_{j j}$ are the two smallest elements of the main diagonal. (Received November 13,1956.)

\section{Bailey Brown and N. M. McCoy (p): Prime ideals in non- associative rings.}

If $A$ and $B$ are ideals or, more generally, any sets of elements of the nonassociative ring $R$, by $A B$ is meant the set of all elements of $R$ of the form $a b, a \in A, b \in B$. Let $\mathfrak{A}$ denote the set of all finite formal nonassociative products of the indeterminates $x_{1}, x_{2}, \cdots$, and let $u=u\left(x_{1}, x_{2}, \cdots, x_{n}\right) \in \mathfrak{A}$. An ideal $P$ in $R$ is $u$-prime if $u\left(A_{1}, A_{2}, \cdots, A_{n}\right) \subseteq P$ implies that some $A_{i} \subseteq P$, where the $A_{i}$ are ideals in $R$. Let $u^{*}=u\left(x_{1}, x_{1}, \cdots, x_{1}\right)$. Then if $u$ contains at least two distinct indeterminates, a $u$-prime ideal is prime and a $u^{*}$-prime ideal is semi-prime, but examples show that the converses need not be true. The theory of the $u$-radical parallels much of the theory of the prime radical in the associative case. In particular, the $u$-radical always coincides with the $u^{*}$-radical. This has been established by Amitsur (Amer. J. Math. vol. 76 (1954) pp. 126-136) for the case in which $u=x_{1} x_{2}$, and is well known in the associative case. Various properties of these radicals are obtained, including the relation to a certain type of nilpotence and to some other radicals. For example, a primitive ideal is $u$-prime for each $u \in \mathfrak{A}$, and hence the Jacobson radical is $u^{*}$-prime for each $u \in \mathfrak{A}$. (Received November 13, 1956.)

154. A. T. Butson: Simply ordered ideal preserving groups. Preliminary report.

In a recent paper (Ideals in partially ordered sets, Amer. Math. Monthly vol. 61 (1954) pp. 223-234), Frink proposed a definition of ideal in a poset, and suggested the possibility of generalizing ordered algebraic systems by requiring the algebraic operations to preserve ideals rather than the order relation itself. The generalization obtained of a simply ordered group is as follows. A simply ordered ideal preserving group (soip-group) $G$ is a system which is (i) simply-ordered, (ii) a group, in which (iii) $J$ an ideal implies $a+J+b$ is either an ideal or a dual ideal for all $a, b$ in $G$. The only nontrivial finite soip-group is the group $B$ containing two elements $b, e$ where $b+e$ $=e+b=b, b+b=e+e=e$, and $b<e$. Every infinite soip-group $G$ is either (1) a simply ordered group, or (2) isomorphic algebraically to the direct sum $B \oplus F$ and orderwise to the ordinal sum $\widetilde{F} \oplus F$, where $F$ is some simply ordered group and $\widetilde{F}$ its dual. (Received November $13,1956$.

155t. Bomshik Chang and S. A. Jennings: On certain free groups generated by two matrices.

Let $F_{\alpha, \beta}=\{A, B\}$ where $A=\left(\begin{array}{ll}1 & \alpha \\ 0 & 1\end{array}\right), B=\left(\begin{array}{ll}1 & 0 \\ \beta & 1\end{array}\right), \alpha$ and $\beta$ being complex numbers. We prove that if either (i) $|\alpha \beta| \geqq 4$ or (ii) $I(\alpha \beta) \geqq 2$ (where $I(z)$ is the imaginary part of $z$ ), then $F_{\alpha, \beta}$ is a free group generated by $A$ and $B$. If $A_{1}=\left(\begin{array}{ll}1 & \alpha \\ 0 & 1\end{array}\right), A_{2}=\left(\begin{array}{ll}1 & \alpha i \\ 0 & 1\end{array}\right), B_{1}=\left(\begin{array}{ll}1 & 0 \\ \alpha & 1\end{array}\right)$, $B_{2}=\left(\begin{array}{cc}1 & 0 \\ \alpha i & 1\end{array}\right)$ with $|\alpha| \geqq 2$, then $F=\left\{A_{1}, A_{2}, B_{1}, B_{2}\right\}$ is the free product of the abelian groups $F_{A}=\left\{A_{1}, A_{2}\right\}$ and $F_{B}=\left\{B_{1}, B_{2}\right\}$. These results generalize those of Sanov 
[Doklady Akad. Nauk SSSR. (NS) vol. 57 (1948) 657-659] and Brenner [Bull. Amer. Math. Soc. vol. 62 (1956) 149]. (Received November 5, 1956.)

156. H. S. M. Coxeter: Groups generated by unitary reflections of period two.

Among the unitary groups generated by reflections [G. C. Shephard and J. A. Todd, Canadian J. Math. vol. 6 (1954) pp. 274-304], those whose generators are involutory are found to belong to a single family. Such a group is denoted by a symbol $\left[p q r^{l}\right]^{m}$, where $p+q+r$ is the number of generators while $l$ and $m$ are the periods of certain products of generators. For instance, $\left[\begin{array}{lll}1 & 1 & 2^{4}\end{array}\right]^{4}$, in unitary 3 -space, is an infinite group which transforms the origin into a lattice consisting of the points whose coordinates are mutually congruent $(\bmod c)$, while their sum is congruent to zero $(\bmod \bar{c})$, in the domain of algebraic integers generated by the roots, $c$ and $\bar{c}$, of the equation $x^{2}+x+2=0$. The corresponding lattice in real Euclidean 6-space represents a new extreme senary quadratic form, recently discovered by E. S. Barnes. (Received November 13, 1956.)

157. R. B. Crouch (p) and W. R. Scott: Normal subgroups of monomial groups.

Let $U$ be a set, $H$ a group, $B$ an infinite cardinal, and $B^{+}$the successor of $B$. Let $o(U)=B$, where $o(U)$ means the number of elements of $U$. The set of monomial substitutions on the elements of $U$ with coefficients in $H$ forms a group $\sum\left(H ; B, B^{+}, B^{+}\right)$. The set $\sum(H ; B, C, D)$ of substitutions of the form $y=v s$, where $v$ is a multiplication with less than $C$ nonidentity factors, $s$ is a permutation that permutes less than $D$ elements of $U$, is a subgroup. The normal subgroups of $\sum\left(H ; B, \aleph_{0}, \aleph_{0}\right)$ are known (R. B. Crouch, Trans. Amer. Math. Soc. vol. 80 (1955) pp. 187-215). This paper extends the results by determining all the normal subgroups of $\sum\left(H ; B, \aleph_{0}, C\right)$ for $\aleph_{0}<C \leqq B$. (Received November 5,1956 .)

158. R. L. Davis: Torsion structure in Engel modules of arbitrary exponent.

Following the discovery of torsion in the Engel module of exponent 5 (Bull. Amer. Math. Soc. Abstract 62-6-605), this paper studies structure questions for Engel modules of arbitrary exponent. Let an Engel module $E$ on 3 or more generators have exponent $m$ (prime or composite). Then the submodule of $E$ consisting of forms of degree $m-2$ in $x, 1$ in $y$ and 1 in $z$ has the module structure of $Z_{p}+Z+\cdots+Z$ $\left(m-3\right.$ copies of $Z$ ) if $m=p^{k}$ is a prime power (here $Z$ is the integers and $Z_{p}$ the integers $\bmod p$ ); for any other $m$ it is simply the direct som of $m-3$ copies of $Z$. The methods are applied to study Engel modules of exponent 4 in all dimension submodules. (Received November 13, 1956.)

159t. John De Cicco: Some theorem concerning commutative rings with unit which admit involutorial automorphisms.

Characterizations of quadratic extensions $Q$ of a commutative ring $X$ with unit 1 in which $2=1+1$, is regular in $X$, are obtained within isomorphisms. Any such ring $Q$ admits an involutorial automorphism. Applications are given to the case where $X$ is a field $F$. The elements of such a ring $Q$ may be represented as points of a z-plane. This plane is a two dimensional vector space relative to the ring $X$. Inner product and norm are studied. Under certain general conditions, isotropic coordinates can be 
introduced in this z-plane. Relations are developed between isotropics and residue classes relative to certain principal ideals. Polynomial forms as well as polynomial functions form rings which admit involutorial automorphisms. Theorems are developed concerning the roots of an algebraic equation over such a ring $Q$. (Received September 10, 1956.)

160. Philip Dwinger: $A$ note on direct sums and direct products in completely modular complete lattices.

Let $L$ be a completely modular complete lattice (Kurosh, Theory of Groups, Vol. II). A product $\prod_{\alpha \in A} x_{\alpha}, x_{\alpha} \in L$ is called a direct product if $x_{\alpha}+x_{\alpha}^{*}=1$ for every $\alpha \in A$, where $x_{\alpha}^{*}=\prod_{\beta \in A, \beta \neq \alpha} x_{\beta}$. A direct product is called regular if for every $A_{1} \subset A, \prod_{\alpha \in \Lambda_{1}} x_{\alpha}+\prod_{\alpha \in A-A_{1}} x_{\alpha}=1$. In the present paper it is shown that if $\left\{x_{\alpha}\right\}, \alpha \in A$, is a set of elements of $L$ the sum of which is a direct sum and which is equal to 1 and $x_{\alpha}^{\prime}=\sum_{\beta \in A, B \neq \alpha} x_{\beta}$ for every $\alpha \in A$, then the product of the set $\left\{x_{\alpha}^{\prime}\right\}$ is a regular direct product which is equal to 0 and $x^{\prime *}=x_{\alpha}$ for every $\alpha \in A$. Conversely if $\left\{x_{\alpha}\right\}, \alpha \in A$, is a set of elements the product of which is a direct product (not necessarily regular) and which is equal to 0 , then the sum of the set $\left\{x_{\alpha}^{*}\right\}$ is a direct sum and $x_{\alpha}^{* \prime} \leqq x_{\alpha}$ for every $\alpha \in A$, while $x_{\alpha}^{* \prime}=x_{\alpha}$ for every $\alpha \in A$ if and only if $\sum_{\alpha \in A} x_{\alpha}^{*}=1$. The first of these results was proved by the author in a previous paper (Indag. Math. vol. 18, p. 435) for a smaller class of lattices (see also P. Dwinger and $\mathrm{J}$. de Groot, On the axioms of Baer and Kurosh in modular lattices, to appear in Ind. Math. vol. 18). The theorems are applied to the lattice of congruence relations of a universal algebra any two congruence relations of which are permutable and with a selected one-element subalgebra. (Received November 7, 1956.)

161. B. M. Dwork: A model for multiplicative characters of local number fields.

Let $k$ be a $p$-adic number field, i.e. a finite extension of a rational $p$-adic field $Q$. The characters of the multiplicative group, $1+p$ are described in terms of additive characters and polynomials, $F_{r}(x)=\sum_{j=1}^{p^{r-1}} x^{i} / j$. Let $\theta$ be a (continuous) additive character of $Q$ and let $\Theta=\theta \circ S_{k / Q}$. An infinite sequence $A=\left(a_{i}\right)_{i=0}^{\infty}$ of elements of $k$ is said to be an $n$-sequence if for all $r \geqq 0, \Theta\left(\sum_{i=0}^{r} a_{i} x^{p^{r-i}} / p^{r-i}\right)=1$ for $p^{r}$ ord $x \geqq n$. For $p^{r} \operatorname{ord}_{\mathfrak{p}} x \geqq n$, let $X_{A}(1-x)=\Theta\left(\sum_{i=0}^{r-1} a_{i} F_{r-i}(x)\right)$. The basic result is that $A \rightarrow X_{A}$ is a well defined homomorphism of the group (under componentwise addition) of $n$ sequences onto the character group of $(1+p) /\left(1+p^{n}\right),(n \geqq 1)$, and the kernel is the set of all $A$ such that $\left(0, a_{0}, a_{0}+a_{1}, a_{0}+a_{1}+a_{2}, \cdots\right)$ is also an $n$-sequence. The theory is applied to complete the explicit reciprocity law for cyclic, ramified extensions of $k$ of degree $p$ (cf. MacKenzie and Whaples, Amer. J. Math. vol. 78 (1956) pp. 473486). This is done by determining an $A$ such that $X_{A}$ is a nontrivial character of the Galois group. The treatment hinges upon the self-duality of $k$ as an additive group. (Received October 15, 1956.)

\section{C. C. Faith: Normal extensions in which every element with} nonzero trace is a normal basis element.

Let $K / k$ be a normal extension, where $K=K_{1} \times K_{2}$ over $k$, for subfields $K_{1}, K_{2}$, where $K_{1} \neq k$. Let $G$, $\mathcal{G}$, and $\mathcal{H C}$ denote the Galois groups of $K / k, K / K_{2}$, and $K_{2} / k$, resp. $w \in K$ (resp. $K_{2}$ ) is a normal basis element (n.b.e.) in $K / k$ (resp. $K_{2} / k$ ) if the set $\left\{w^{s}\right\}, S \in G$ (resp. $\mathcal{H C}$ ), forms a basis of $K / k$ (resp. $K_{2} / k$ ). The trace of $w$ in $K / K_{2}$ is $T_{K / K_{2}}(w)=\sum_{s \in \mathcal{G}} w^{s}$. The equivalence of (1) and (2) is demonstrated: (1) $T_{K / K_{2}}(w)$ 
is a n.b.e. in $K_{2} / k$ implies $w$ is a n.b.e. in $K / k ;(2) k$ has prime characteristic $p$ and $K_{1} / k$ has degree $p^{\text {s. Letting }} K_{2}=k$ so that $K=K_{1}$, one sees that (2) is equivalent to: (1') $T_{K / k}(w) \neq 0$ implies $w$ is a n.b.e. in $K / k$. Perlis [Theorem 1, Duke Math. J. vol. 9 (1942) pp. 507-517] proved (2) implies (1') assuming $K / k$ is cyclic. Thus, this result is extended to the case that $k$ has prime characteristic $p$, and $K / k$ is an arbitrary normal extension of degree $p^{e}$. Moreover, our results show that these are the most general normal extensions satisfying $\left(1^{\prime}\right)$. Let $G(k)$ be the group algebra defined by $G$ and $k$. The elements of $G$ are right operators on $G(k)$ and $K$. An (operator) module isomorphism between $G(k)$ and $K$ enables us to apply structure theorems for $G(k)$ to obtain our results. (Received November 13, 1956.)

163t. C. C. Faith: On decompositions of certain subgroups of abelian groups. Preliminary report.

(A) If $S$ is any subgroup of a finite, $p$-primary, abelian, additive group $G$ satisfying $p^{\alpha} G \geqq S \geqq p^{\alpha+1} G$, then there exists a decomposition of $G$ into the direct sum of cyclic subgroups $G_{i}$ such that $S$ is the direct sum of the subgroups $S \cap G_{i}$. The proof is obtained by the proof of (A) when $\alpha=0$ by induction on the order of $G$, followed by an induction on $\alpha$. If $K / k$ is an abelian extension, application of (A) via the Galois Theory yields new results concerning factorizations over $k$ of certain intermediate fields of $K / k$. In the remainder, $M$ always denotes a module over a discrete valuation ring $R$ with unique prime $p$. A direct calculation shows: (B) If $S$ is any submodule of $M$ obeying (1) $p^{\alpha} M \geqq S \geqq p^{\alpha+1} M$, then $S$ is regular (in the sense of Vilenkin, i.e., $p^{n+k} S \cap p^{k} M=p^{n}\left(S \cap p^{k} M\right)$, for any $\left.n, k\right)$. A characterization of regularity in Exercise 78(b) in Kaplansky's "Infinite abelian groups," produces a kind of generalization of (A): (C) If $M$ has bounded order, and if $S$ is any submodule of $M$ satisfying (1), then there exists a basis $\left\{x_{i}\right\}$ of $M$ such that $\left\{r_{i} x_{i}\right\}$ is a basis for $S$, for suitable $r_{i}$ in $R$ (also see Ex. 79, loc. cit., in this connection). (Received November 13, 1956.)

\section{Walter Feit: Integral representations of finite groups.}

Let $A$ be the group algebra of a finite group $G$ of order $g$ over the field $K$ of $g$ th roots of unity. Let $S$ be a set of prime ideals in $K, D$ the ring of elements in $K$ which are local integers at each prime in $S$, and let $R$ be the $D$ module whose basis consists of the elements of $G$. Corresponding to the decomposition of $R$ into a direct sum of indecomposable left ideals (Bull. Amer. Math. Soc. Abstract 63-1-4) there is a decomposition of $A$ into a direct sum of left ideals, each summand of $A$ corresponds to representation $U$ of $G$ with trace $\Phi$. It is shown that each $\Phi$ is a character of $G$ which vanishes for all $S$-singular elements, i.e. elements which are $p$-singular for some $p$ which has a divisor in $S$. The number of $\Phi$ 's equals the number of $S$-regular classes of $G$. If $S$ consists of only one prime ideal $p$, then the $\Phi$ 's are the principal indecomposable characters of $G \bmod p$. The dual basis of the $\Phi$ 's corresponds to the irreducible characters $\bmod p$. Various relationships involving generalized Cartan invariants and decomposition numbers are obtained. (Received October 24, 1956.)

\section{J. K. Goldhaber: The nonexistence of certain finite projective planes possessing a polarity.}

Using results of $\mathrm{R}$. Baer (Polarities in finite projective planes, Bull. Amer. Math. Soc. vol. 52 (1946) pp. 77-93) concerning the absolute points of a polarity, a canonical form for the incidence matrix of a projective plane possessing a polarity and having an odd number of points on a line is derived. On the basis of this canonical form and 
with the aid of the method of R. H. Bruck and H. J. Ryser (The nonexistence of certain finite projective planes, Canadian J. Math. vol. 1 (1949) pp. 88-93) the following theorem is proved: If $n \equiv 0(\bmod 4)$ and if a prime of the form $4 k+3$ divides the squarefree part of $n$ then a projective plane which possesses a polarity and which has $n+1$ points on a line does not exist. (Received October 24, 1956.)

\section{Juris Hartmanis: A note on the lattice of geometries.}

It has been shown (Proc. Amer. Math. Soc. vol. 7 (1956) pp. 571-577) that every finite lattice can be embedded in the lattice of all geometries on a finite set. Since the corresponding problem for partition lattices is still an unsolved problem, the common properties of these lattices are investigated. (cf. ORE, Duke Math. J. vol. 9 (1942) pp. 573-627). It is shown that they are complemented, have only trivial homomorphisms and that the group of automorphisms is a symmetric group. Finally, it is noted that the quotient lattices of the partition lattices are isomorphic to direct products of partition lattices. The corresponding result does not hold for the lattice of geometries. (Received November 9, 1956.)

167. Edwin Hewitt (p) and H. S. Zuckerman: The irreducible representations of a semigroup related to the symmetric group.

Let $\mathfrak{T}_{n}$ be the semigroup of all single-valued mappings of a set consisting of $n$ elements into or onto itself: for $f, g \in \mathfrak{I}_{n}, f g(x)=f(g(x))$. An explicit method is given for computing all irreducible representations of $\mathfrak{I}_{n}$ by semigroups of complex matrices. There are several steps. Let $\mathfrak{B}_{p}=\left\{f: f \in \mathfrak{I}_{n}\right.$, range of $f$ contains just $p$ elements $\}$ $(p=1,2, \cdots, n)$. For every irreducible representation $M$ of $\mathfrak{T}_{n}$, there is a $p$ such that $M(f)=0$ for $f \in \bigcup_{j-1}^{p-1} \mathfrak{B}_{j}$ and $\{M(f)\}_{f \in \mathfrak{B}_{p}}$ is irreducible. Every $M$ is completely determined by its values on $\mathfrak{B}_{p}$. Every irreducible representation of $\mathfrak{B}_{p} \cup\{z\}$ (this is $\mathfrak{B}_{p}$ with a zero added to make it a semigroup) can be extended to a representation of $\mathfrak{T}_{n}$. The irreducible representations of $\mathfrak{B}_{p} \cup\{z\}$ are computed explicitly in terms of the irreducible representations of the symmetric group $\mathfrak{S}_{p}$. (Received October 22, 1956.)

168t. J. H. Hodges: Weighted partitions for hermitian matrices over a finite field.

Let $q=p^{n}, q$ odd, and suppose that $\theta \in G F\left(q^{2}\right), \theta^{2}=\nu \in G F(q)$, but $\theta \notin G F(q)$. Then $\alpha=a+b \theta \in G F\left(q^{2}\right)$ if $a, b \in G F(q) . \bar{\alpha}=a-b \theta$ is called the conjugate of $\alpha$. If $A=\left(\alpha_{i j}\right)$ is a square matrix, $\alpha_{i j} \in G F\left(q^{2}\right)$, let $A^{*}=\bar{A}^{\prime}=\left(\alpha_{i j}\right)^{\prime}$ where the prime denotes transpose. Then $A$ is said to be hermitian if and only if $A^{*}=A$. For $a \in G F(q)$, let $e(a)=e^{2 \pi i t(a) / p}$ where $t(a)=a+a^{p}+\cdots+a^{p^{n-1}}$. If $A=\left(\alpha_{i j}\right), \alpha_{i j} \in G F\left(q^{2}\right)$ is hermitian, then $\sigma(a)=\sum_{i \alpha_{i i}}$, the trace of $A$, is a number of $G F(q)$. Consider the sum $S=S(B, X, A)=\sum e\left\{\sigma\left(X^{*} U+U^{*} X\right)\right\}$, where $B, X, A$ and $U$ are matrices over $G F\left(q^{2}\right), A$ is hermitian and nonsingular of order $m, B$ is hermitian of order $t, X$ and $U$ are $m \times t$ and the sum is over all $U$ satisfying $U^{*} A U=B$. If $X=0, S$ is the number of solutions $U$ of $U^{*} A U=B$ which is given in Representations by hermitian forms in a finite field (L. Carlitz and J. H. Hodges, Duke Math. J. vol. 22 (1955) pp. 393-405). In the present paper it is shown that $S$ can be expressed in terms of certain Kloosterman sums defined for hermitian matrices over $G F\left(q^{2}\right)$. A number of the properties of these Kloosterman sums are also given. The analogous sums for symmetric, skew, and general square matrices over $G F(q)$ have been considered previously by the author. (Received November 13, 1956.) 
169. D. R. Hughes: Generalized incidence matrices over group algebras.

Let $\pi$ be a $(v, k, \lambda)$ configuration, $\&$ a collineation group of $\pi$, and let $Q$ be the group algebra of $\$ 3$ over an appropriate field $F$ (e.g., $F$ can be any field of characteristic zero). Then there exist diagonal matrices $C_{1}, C_{2}$ with entries from $F$, and (square) matrices $D, B_{1}, B_{2}$ with entries from $Q$, such that $D C_{1} D^{*}=B_{1}, D^{*} C_{2} D=B_{2}$, where $D^{*}$ is the conjugate transpose of $D$. In the cases of interest (e.g., $\&$ has prime order), the $B_{i}$ and $C_{i}$ are completely known. These matrix equations imply the author's earlier results on generalized incidence matrices with rational entries, but also yield new results, such as: if $\pi$ is a projective plane of order $n \equiv 2(\bmod 4), n \neq 2$, then $\pi$ possesses no collineations of even order. (Received October 19, 1956.)

\section{R. E. Johnson: Rings with unique addition.}

A ring $R$ is said to have unique addition if every multiplicative mapping of $R$ onto a ring $S$ is also additive. Rickart has shown (Bull. Amer. Math. Soc. vol. 54 (1948) pp. 758-764) that a semi-simple ring $R$ satisfying certain minimum conditions has unique addition. The present paper extends Rickart's results to rings with zero singular ideal (Proc. Amer. Math. Soc. vol. 2 (1951) p. 894). (Received November 5, 1956.)

\section{C. W. Kohls: Ideals in rings of continuous functions. III.}

Let $C(X)$ be the ring of all continuous real functions on a completely regular Hausdorff space $X ; \beta X$, the Stone-Cech compactification of $X ; v X$, the largest subspace of $\beta X$ over which all $f C(X)$ can be extended; $N^{p}=\{f \in C(X): f=0$ on some $X$-nbhd. of $p\}$; and $M^{\prime} p=\{f \in C(X)$ : the zero-set of $f$ meets every deleted nbhd. of $p\}$, if $p$ is a nonisolated point of $X,=M^{p}$ otherwise. For any $p \in \beta X, p$ is a $\beta F$-point with respect to $X$ if whenever $f \in C(X)$ is extendable to $p$ and vanishes there, then $f \geqq 0$ or $f \leqq 0$ on some $X$-nbhd. of $p$. (1) If $P$ is any prime ideal of $C(X)$, then $C(X) / P$ is an ordered integral domain containing the reals. Let $p \in \beta X$ be the unique point such that $P \supseteq N^{p}$. Then $C(X) / P$ has infinitely large elements iff $p \notin v X$. (2) Let $p$ be a $\beta F$-point. Then for each pair of subsets $D, D^{\prime}$ of $C(X) / N^{p}$ of order type $\omega, \omega^{*}$ resp., with $D<D^{\prime}$, there is an element $\delta \in C(X) / N^{p}$ such that $D<\delta<D^{\prime}$. The ring $C(X) / N^{p}$ has no countable cofinal subset iff $p \notin v X$. (3) An example is given of a space $X$ and a $\beta F$-point $p \in \beta X-v X$ such that the positive subset of $C(X) / N^{p}$ has countable coinitial subset. (This corrects an assertion of Bull. Amer. Math. Soc. Abstract 62-6-622.) (4) Let $p \in X$ be a $\beta F$-point. Then every proper open subinterval of $C(X) / M^{\prime p}$ is an $\eta_{1}$-set iff $M^{\prime p} \neq M^{p}$. (Received December 10,1956.)

\section{2t. C. W. Kohls: Ideals in rings of continuous functions. IV.}

For any $p \in \beta X, p$ is a $V$-point if $C(X) / N^{p}$ is a valuation ring (see previous abstract for terminology); equivalently, if every finitely generated ideal of $C(X)$ containing $N^{p}$ is principal. If $p$ is a $V$-point it is a $\beta F$-point. Several sufficient conditions that a $\beta F$-point be a $V$-point have been obtained; whether every $\beta F$-point is a $V$ point is undetermined. Let $p$ be a $V$-point which is not a $P$-point. For each $a \in M^{p} / N^{p}$, $a>0$, we call $Q^{a}=\left\{b \in C(X) / N^{p}:|b| \leqq a^{r}\right.$ for some real $\left.r>0\right\}$ an upper ideal, and $Q_{a}=\left\{b \in C(X) / N^{p}:|b|<a^{r}\right.$ for all real $\left.r>0\right\}$ a lower ideal. (1) $Q^{a}$ and $Q_{a}$ are prime ideals. (2) A prime ideal $I$ of $C(X) / N^{p}$ has an immediate successor $J$ under set inclusion iff for some $a \in M^{p} / N^{p}$, we have $I=Q_{a}$ and $J=Q^{a}$. (3) If $Q^{a} \subseteq Q_{b}$, there exists 
$c \in C(X) / N^{p}$ such that $Q^{a} \subseteq Q_{c} \subseteq Q^{c} \subseteq Q_{b}$. (4) Let $I$ be an ideal of $C(X)$ such that $I / N^{p}$ is a lower ideal. Then neither $I$ nor $I / N^{p}$ is countably generated. (5) We call an ideal $I$ a $\mathbb{B}$-ideal if whenever $f$ and $g$ have the same zero-sets and $g \in I$, then $f \in I$. If $I$ is a 3 -ideal containing $N^{p}$, then $I / N^{p}$ is not a lower ideal, nor an upper ideal if $I \neq N^{p}$. (6) There are uncountably many prime ideals in $C(X) / N^{p}$. (7) Given $a \in M^{p} / N^{p}, a>0$, for each $b \in Q^{a}-Q_{a}$, we have $b=h a^{*}$ or $a^{s}=h b$ for some real $s>0$ and $h \in C(X) / N^{p}-Q^{a}$. (Received December 10,1956.)

\section{3t. Georg Kreisel: Hypotheses on algebraically closed extensions.}

Let $P\left(a_{1}, \cdots, a_{m}, x_{1}, \cdots, x_{n}, t_{1}, \cdots, t_{p}\right)[P(\mathfrak{a}, \mathfrak{x}, \mathfrak{t})]$ be a formula built up of the field operations and the variables indicated, with the property: in the (commutative) field considered, (a) if $(\mathfrak{x})\left[P\left(\mathfrak{a}, \mathfrak{x}, \phi^{(1)}\right) \bigvee \cdots \vee P\left(\mathfrak{a}, \mathfrak{x}, \phi^{(k)}\right)\right]$ for algebraic functions $\phi_{i}^{(j)}$, then (b) (x) $P(\mathfrak{a}, \mathfrak{x}, \tau)$ for suitable polynomials $\tau_{i}\left(a_{1}, \cdots, a_{m}, x_{1}, \cdots, x_{n}\right)$. Suppose $H$ is a hypothesis formulated in the first-order predicate calculus from which $(\mathfrak{x})(E \mathfrak{t}) P(\mathfrak{a}, \mathfrak{x}, \mathfrak{t})$ follows by (a necessarily finite subset of) the axioms for algebraically closed fields (ACF); then (b), where the construction of $\tau$ is effective provided the step from (a) to (b) is effective.-By ACF, $H \leftrightarrow H_{0}, H_{0}$ quantifier-free (Tarski), where the axioms $(E z)\left(z^{n}+c_{1} z^{n}+\cdots+c_{n}=0\right)$ are replaced by $p_{n}^{n}+c_{1} \rho_{n}^{n-1}+\cdots+c_{n}=0$, $\rho_{n}\left(c_{1}, \cdots, c_{n}\right)$ being new function symbols (for algebraic functions). By Hilbert's first $\epsilon$-theorem, if $(\mathfrak{c})(E \mathrm{t}) P(\mathfrak{a}, \mathfrak{x}, \mathfrak{t})$ by (quantifier-free) ACF and $H_{0}$, then $P(\mathfrak{a}, \mathfrak{x}$, $\left.\psi^{(1)}\right) \bigvee \cdots \vee P\left(\mathfrak{a}, \mathfrak{x}, \psi^{(k)}\right)$, where the $\psi$ are made up of the field operations and $\rho_{2}, \cdots, \rho_{N}$ : apply the assumption on $P$. -Example. For $P$ take $1=t_{1} f_{1}+\cdots+t_{p} f_{p}$ where $f_{i}$ is the general polynomial in $n$ variables of degree $d$ with coefficients among the $\mathfrak{a}$; for $H$ take $\left(x_{1}\right) \cdots\left(x_{n}\right)\left(f_{1} \neq 0 \vee \cdots \vee f_{p} \neq 0\right)$ (meaning that in some, hence every, AC extension of the field generated by $a_{1}, \cdots, a_{m}$, the $f$ do not have a common zero). Then the conditions on $P$ apply, yielding Hilbert's Nullstellensatz with a primitive recursive bound in $n, p$, and $d$ for the degrees of $\tau_{i}$, independent of the field of coefficients. (Received November 13, 1956.)

\section{Georg Kreisel: Hilbert's 17th problem. I.}

Let $f\left(a_{1}, \cdots, a_{m}, x_{1}, \cdots, x_{n}\right)$ be the general rational function (with numerator and denominator) of degree $d$ in $n$ variables, with coefficients $a$ in a real commutative field $P$ in which every positive element is the sum of $\leqq r$ squares. There are rational functions $g_{i}\left(a_{1}, \cdots, a_{m}, x_{1}, \cdots, x_{n}, b_{1}, \cdots, b_{q}\right), i \leqq \lambda(n, d, r)$, of degree $\leqq \lambda(n, d, r)$ in the variables shown, with the following property: if $f \nless 0$ in some real closed extension of the field generated by $a_{1}, \cdots, a_{m}$, then there are $\bar{b}_{1}, \cdots, \bar{b}_{q}$ in this field such that $f=\sum_{1}^{\lambda} \bar{g}_{i}^{2}, \lambda$ primitive recursive (and independent of $P$ ). This strengthens Robinson's result [Math. Ann. vol. 130 (1955) pp. 257-271] where $P$ was assumed to be real closed and $\lambda$ was shown to be general recursive. $f \nless 0$, i.e. $\left(x_{1}\right) \cdots\left(x_{n}\right)$ $\cdot(E t)\left(f=t^{2}\right) \leftrightarrow\left(E b_{1}\right) \cdots\left(E b_{q}\right) H_{0}\left(a_{1}, \cdots, a_{m}, b_{1}, \cdots, b_{q}\right), H_{0}$ quantifier-free, for realclosed fields, i.e. from (i) field axioms, (ii) $\left(u_{1}\right) \cdots\left(u_{k}\right)\left(u_{1}^{2}+u_{2}^{2}+\cdots+u_{k}^{2}+1 \neq 0\right)$, $k$ depending on $n, d$, and $r$, (iii) $(x)(E y)\left(x=y^{2} v-x=y^{2}\right)$ and (iv) $\left(v_{1}\right) \cdots\left(v_{2 n+1}\right)$ - $\left(E w_{n}\right)\left(w_{n}^{2 n+1}+v_{1} w_{n}^{2 n}+\cdots+v_{2 n+1}=0\right)$. (iii) and (iv) are made quantifier-free by replacing $y$ by $\zeta(x), w_{n}$ by $\zeta_{n}\left(v_{1}, \cdots, v_{2 n+1}\right)$. Since $\left(x_{1}\right) \cdots\left(x_{n}\right)\left(E t_{1}\right) \cdots\left(E t_{k}\right)$ $\cdot\left(f=t_{1}^{2}+\cdots+t_{k}^{2}\right)$ follows from (i), (iii) and the negation of (ii), it follows from (i), (iii), (iv) and $H_{0}$. By Hilbert's first $\epsilon$-theorem, as in the preceding abstract, $(*) f=\sum_{j=1}^{k} \phi_{j 1}^{2} \vee \cdots \vee f=\sum_{j=1}^{k} \phi_{N j}^{2}$, with $\phi$ composed of the field operations, $\zeta$ and $\zeta_{n}$. (*) can be contracted to $f=\sum \Phi_{i}^{2}$, and $\zeta$ and $\zeta_{n}$ eliminated. (Received November $13,1956$. 


\section{5t. Georg Kreisel: Hilbert's 17th problem. II.}

A simpler construction of $\phi$ and $\lambda$ (of the preceding abstract) is obtained by applying the methods of JSL 16 (1951) 241-267, to Artin's solution of Hilbert's problem [Abh. Math. Seminars Hamburg Univ. vol. 5 (1927) pp. 100-115]. A rough calculation shows that $\lambda(2, d, r)$ is of order $v(7, d)$ where $v(0, n)=n, v(m+1, n)=2^{v(m, n)}$. $f$ and its Sturm chain $f^{(k)}$ are formally factorized into (at most) quadratic factors, $f^{(k)}=h^{(k)}\left(x_{2}, \cdots, x_{n}\right) \prod\left[\left(x_{1}-\alpha^{(k)}\right)^{2}+\beta_{i}^{(k)}\right]$ in an extension of $P$ by terms built up of $\zeta$ and $\zeta_{p}$. Elementary arguments show that either $f$ or -1 can be represented as (different) sums of squares in each set of extensions of the following form: $\left( \pm h^{(k)}\right)^{1 / 2}$; $\left( \pm \beta_{i}^{(k)}\right)^{1 / 2}$ and, for each adjunction of $\left(-\beta^{(a)}\right)^{1 / 2},\left(-\beta_{j}^{(b)}\right)^{1 / 2},\left\{ \pm\left[\left(\alpha_{i}^{(a)} \pm\left(-\beta_{i}^{(a)}\right)^{1 / 2}\right)\right.\right.$ $\left.\left.-\left(\alpha_{j}^{(b)} \pm\left(-\beta_{i}^{(b)}\right)^{1 / 2}\right)\right]\right\}^{1 / 2}$ (all possible orderings of the "real" zeros of $\left.f^{(k)}\right)$. These adjunctions can now be eliminated, as in I. Sample: Suppose $s^{1 / 2}$ and $(-s)^{1 / 2}$ are both adjoined (separately, cf. $\zeta$ in I), $-1=\sum\left(a_{n}+b_{n} s^{1 / 2}\right)^{2}$, and $-1=\sum\left(c_{n}+d_{n}-(s)^{1 / 2}\right)^{2}$; (i) if $(N)$, i.e. $\sum a_{n} b_{n} \neq 0$ or $\sum c_{n} d_{n} \neq 0$, either $-1=\sum\left[a_{n}-2^{-1} b_{n}\left(1+\sum a_{n}^{2}+s \sum b_{n}^{2}\right)\right.$ - $\left.\left(\sum a_{n} b_{n}\right)^{-1}\right]^{2}$ or $-1=\sum\left[c_{n}-2^{-1} d_{n}\left(1+\sum c_{n}^{2}-s \sum d_{n}^{2}\right)\left(\sum c_{n} d_{n}\right)^{-1}\right]$ where, without loss of generality, $a^{-1}=0 \leftrightarrow a=0$, (ii) if not $N$, and $\sum b_{n}^{2}+\sum d_{n}^{2} \neq 0(Z)$, then -1 $=\left(\sum a_{n}^{2} \sum d_{n}^{2}+\sum c_{n}^{2} \sum b_{n}^{2}\right)\left(\sum b_{n}^{2}+\sum d_{n}^{2}\right)^{-1}$, (iii) if not $N$ and not $Z$, then $\sum b_{n}^{2}=0$ and $-1=\sum a_{n}^{2}$, or $\sum b_{n}^{2} \neq 0$ and $-1=\left(\sum d_{n}^{2}\right)\left(\sum b_{n}^{2}\right)^{-1}$. Since $-1=a$ or $-1=b$ implies $-1=a+b+a b$, cases (i)-(iii) can be recombined. The elimination of $\zeta_{p}\left(\alpha_{1}, \cdots\right.$, $\alpha_{2 p+1}$ ) proceeds similarly by cases, according to all possible occurrences of simple and multiple factors of $x^{2 p+1}+\alpha_{1} x^{2 p}+\cdots+\alpha_{2 p+1}$. (Received November 13, 1956.)

\section{J. B. Kruskal: Group invariants and the free calculus.}

Suppose $G$ is a group given by a presentation $\left(x_{1}, \cdots, x_{n} / r_{1}, \cdots, r_{n}\right)$. Let $X$ be the free group on the $x$ 's, let $R$ be the consequence of the $r$ 's, and let $\phi: X \rightarrow X / R \approx G$ be the natural map, so the sequence $1 \rightarrow R \rightarrow X \rightarrow G \rightarrow 1$ is exact. Let $J G$ be the integral group ring, let $o: J G \rightarrow J$ be the coefficient-sum homomorphism, and let $\mathbb{B}$, called the fundamental ideal, be the kernel of $o$. As shown by R. H. Fox, partly in The free differential calculus I and II. Ann. of Math. 1953 and 1954, the "Jacobian matrix" $\phi\left\|D_{j} r_{i}\right\|$ (where $D_{j}$ indicates differentiation with respect to $x_{j}$ ) is a relation matrix for (3), considered as a left-module over $J G$, so the equivalence class of this matrix is an an invariant of $G$. As the relation matrices (or rather, the associated equivalence classes) of the powers (5kk are also invariants of $G$, it is of interest to be able to compute them. With the aid of the operator vectors $\nabla=\left(D_{1}, \cdots, D_{n}\right), \nabla^{2}=\left(D_{1} D_{1}, D_{2} D_{1}, \cdots\right.$, $D_{n} D_{n}$ ), etc., a specific, computable relation matrix for (3)k has been determined. It is not yet clear how useful these invariants will be. (Received November 16, 1956.)

\section{H. T. LaBorde: Bounds for the real parts of the characteristic} roots of a matrix.

In the Journal of Research of the National Bureau of Standards, vol. 48 (1952) P. Stein published a note giving bounds for the real parts of the characteristic roots of a matrix. Two theorems give these bounds in terms of an arbitrary set of positive constants. Though the method is trivial, it will, by judicious selection of values for the arbitrary constants in terms of the elements of the original matrix, give expressions for bounds which are easily obtained and which give better approximations than other existing theorems in many cases. These results can be strengthened so as to be applicable to a larger class of matrices and so as to give refinements of these bounds. This is done in the following theorem: Let $A=\left(a_{r s}\right)$ be an arbitrary square matrix of order $n, M$ the maximum real part and $m$ the minimum real part of the characteristic 
roots of $A$, and let $W_{1}, W_{2}, W_{n-1}, W_{n}$ be the maximum real part, the largest real part save one, the smallest real part save one, and the minimum real part of the main diagonal elements $a_{r r}$ of $A$, respectively. Set $\sigma_{r}=\sum_{\nu=1, p \neq r}^{n}\left|a_{r \nu}+\bar{a}_{\nu r}\right| / 2, \sigma_{p}=\max _{r} \sigma_{r}$, $\sigma_{q}=\max _{r \neq p} \sigma_{r}$. Then $M \leqq \max \left\{W_{1}+\sigma_{q}, W_{2}+\sigma_{p}\right\}$ and $m \geqq \min \left\{W_{n}-\sigma_{q}, W_{n-1}-\sigma_{p}\right\}$. This theorem, together with its applications to real matrices, is further improved by using a theorem of A. Brauer (Duke Math. J. vol. 14 (1947)). (Received December 17, 1956.)

\section{8t. G. F. Leger, Jr.: A note on the cohomology of groups.}

The first three cohomology groups $H^{n}(G, A)(n=1,2,3)$ of a group $G$ with respect to a $G$-module $A$ have well known interpretations. If $G$ is a normal subgroup of a group $\Gamma$ and $\mathrm{A}$ is also a $\Gamma$-module then $G$ operates on $H^{n}(G, A)$. Operations of $\Gamma$ on the interpretations of $H^{n}(G, A)$ are devised which agree with the operations of $\Gamma$ on $H^{n}(G, A)$. The analogue of this for Lie algebras has been done earlier by the author (Bull. Amer. Math. Soc. Abstract 62-4-474). (Received November 13, 1956.)

\section{Saunders MacLane: Obstructions to the extensions of rings.}

The natural homology theory of a ring $\Lambda$ is given by a certain complex $R(\Lambda)$, as described in Abstract 62-6-629. This homology yields a complete treatment for extensions of rings, parallel to the known homological treatment of the extensions of groups. Explicitly a multiplication (Hochschild) of a ring $A$ is a pair of additive mappings $a \rightarrow \sigma a, a \rightarrow a \sigma$ satisfying the associative laws $\sigma(a b)=(\sigma c) b,(a \sigma) b=a(\sigma b)$, and $(a b) \sigma=a(b \sigma)$. All multiplications of $A$ form a ring $M_{A}$, and this is a natural homomorphism $A \rightarrow M_{A}$ mapping $A$ into "inner" multiplications, and with kernel $K_{A} \subset A$, and cokernel the ring $P_{\boldsymbol{A}}$ of multiplication classes. Any extension $E$ of $A$ by a ring $\Lambda$ yields a homomorphism $\theta: \Lambda \rightarrow P_{\Lambda}$. Not every such $\theta$ can be realized by an extension; indeed, $\theta$ has an "obstruction" a certain three-dimensional cohomology class in $H^{8}\left(R(\Lambda), K_{\Lambda}\right)$, and can be realized by an extension exactly then when this obstruction is zero. When there is such an extension the set of possible (inequivalent) extensions is in one-one correspondence with $H^{2}\left(R(\Lambda), K_{\Lambda}\right)$. Finally, every three dimensional cohomology class which annihilates a certain three-dimensional cycle can be realized as an obstruction to a suitable $\theta$. (Received November 1, 1956.)

180. M. D. Marcus (p), B. N. Moyls, and R. Westwick: Some extreme value results for indefinite Hermitian matrices.

Let $A$ be an $n$-square complex indefinite Hermitian matrix. Let $x_{j} j=1, \cdots$, $k \leqq n$ be an orthonormal (o.n.) set and let $\phi=\prod_{1}^{k}\left(A x_{j}, x_{j}\right)$. The extreme values of $\phi$ as the $x_{j}$ run over all o.n. sets are obtained as functions of the eigenvalues. The result for the minimum specializes to the theorem of K. Fan [Amer. Math. Monthly, vol. 60 (1953) pp. 48-50]. Also obtained are the extreme values of $E_{2}\left[\left(A x_{1}, x_{1}\right), \cdots\right.$, $\left.\left(A x_{k}, x_{k}\right)\right]$ where $E_{2}$ is the second symmetric function and $A$ is again indefinite Hermitian. (Received November 6, 1956.)

\section{D. W. Miller: On a theorem of Hölder.}

A classical result of finite group theory, due to Hölder [Math. Ann. vol. 46 (1895) pp. 343-345] is the following. $S_{n}$, the symmetric group on $n$ letters, is a complete group if $n \neq 6$; the group of inner automorphisms of $S_{6}$ has index two in the group of all automorphisms of $S_{6}$. A new proof, suggested by R. H. Bruck, of the existence of an outer 
automorphism of $S_{6}$ is given here. A mapping $\theta$ of $S_{6}$ having the property that (12) $\theta$ $=(12)(36)(45),(23456) \theta=(23456)$ is defined and, without extensive computation, is shown to be an (outer) automorphism of $S_{6}$. (Received November 13,1956.)

\section{J. M. Osborn: Commutative diassociative loops. Preliminary} Report.

A loop is diassociative if every two elements generate a subgroup. A commutative diassociative loop $L$ is a $p$-loop of degree one if every element except the identity has order $p$, so that every simple commutative diassociative loop with elements of finite order is of this type. Let $G$ be a configuration of points and lines such that every two points determine a unique line, and that every line has exactly $n+1$ points on it for some fixed $n$, then $G$ is called a finite geometry of order $n$. There is a 1-1 correspondence between finite geometries of order $p$ and classes of $p$-loops of degree one, which leads to many results on $p$-loops, including the construction of several classes of simple ones. This correspondence "coordinitizes" a projective plane of order $p$ with a $p$-loop of $p^{3}$ elements, and a generalization of this process will coordinitize any projective plane. An application is the determination of all commutative Moufang loops on three generators. (Received November 7, 1956.)

\section{3t. Gordon Pall and Olga Taussky: Application of quaternions to} the representation of a binary quadratic form as a sum of four squares.

Let $r_{d}(n)$ denote the number of representations of the integer $n$ as a sum of $s$ squares of integers, and let $r_{s}(\phi)$ denote the number of representations of the binary quadratic form $\phi$ as a sum of $s$ squares of linear forms with integral coefficients. Write $\phi=e \phi_{1}, \phi_{1}=a x^{2}+2 t_{0} x y+b y^{2}$, where $\left(a, t_{0}, b\right)=1$; set $k=\left(a, 2 t_{0}, b\right)=1$ or 2 . Using simple arguments based almost entirely on the arithmetic of quaternions it is proved that $r_{2}(\phi)=f(e) \cdot 8\left(2+(-1)^{k}\right) \cdot r_{3}(d)$. Here $d=a b-t_{0}^{2}$ and $f(e)$ is a certain factorable function which is explicitly evaluated. (Received November $9,1956$.

\section{E. S. Rapaport: On free groups and their automorphisms.}

A theorem (Whitehead, J. H. C., Ann. of Math. vol. 37 (1936) p. 789) providing an algorithm for determining whether two given sets of $m$ words each in the free group $F_{n}=F\left(a_{1}, \cdots, a_{n}\right)$ are automorphic images of each other, is proved by algebraic means. Let $A$ be the automorphism group of $F_{n}, T_{1}, \cdots, T_{0}=T_{1}^{-1}, \cdots$, a particular set of generators of $A, L(w)$ the sum of the absolute values of the exponents of generators occurring in $w=w\left(a_{1}, \cdots, a_{n}\right) \subset F_{n}$; furthermore, let $a=T_{2} T_{1}$, $T_{1}\left(w_{0}\right)=w_{1}, T_{2}\left(w_{1}\right)=a\left(w_{0}\right)$ subject only to the conditions (1) $L\left(T_{1} w_{0}\right)>L\left(w_{0}\right)$ and (2) $L\left(a w_{0}\right) \leqq L\left(T_{1} w_{0}\right)$. A finite set of words $s_{i} \subset F_{n}$ are found such that $w=\prod_{s_{i} \subset w} s_{i}$ uniquely for every $w$ in $F_{n}$ and, for $T=T_{1}$ and $T=a, L\left(T w_{0}\right)-L\left(w_{0}\right)=L\left(T \pi s_{i}\right)$ $-L\left(\pi s_{i}\right)=\sum s_{i} \subset w_{0}\left(L\left(T s_{i}\right)-L\left(s_{i}\right)\right)$. Let $s_{i}^{\prime}$ be the number of times $s_{i}$ occurs in $w_{0}$. The conditions (1) and (2) and relations of the group $A$ then yield inequalities between certain of the $s_{i}^{\prime}$; these are used to prove the existence of an automorphism $B$ $=T_{i_{k}} \cdots T_{i_{1}}=a$, such that the lengths of the "intermediate words" $T_{i_{1}}\left(w_{0}\right)$, $T_{i_{2}} T_{i_{1}}\left(w_{0}\right), \cdots$ are monotone decreasing up to $i_{h}, h \leqq k$, are unchanged from $i_{h+1}$ to $i_{h^{\prime}}, h \leqq h^{\prime} \leqq k$, and are monotone increasing from $i_{h^{\prime}+1}$ to $i_{k}$. This result is extended to the case where $w_{0}$ is a set of $m$ words and a arbitrary. Several incidental results are given; for example: if $L\left(T w_{0}\right)>L\left(w_{0}\right)$, then $L\left(T^{2} w_{0}\right)>L\left(T w_{0}\right)$ for any $T=T_{i}$. (Received November 9, 1956.) 


\section{J. B. Roberts: On binomial coefficient residues.}

The number of binomial coefficients $\left({ }_{v}^{*}\right), 0 \leqq v \leqq u<n$, which are congruent to $j$, $0 \leqq j \leqq p-1$, modulo the prime number $p$ is denoted by $\theta_{j}(n)$. Systems of simultaneous linear difference equations are given whose solutions yield the $\theta_{j}(n)$. Computations of $\theta_{j}(n)$ for $p=2$ and of $\theta_{j}\left(p^{k}\right)$ for $p=3$ or $5, k \geqq 0$, are carried out. Also it is proved that every prime $p$ divides "most" binomial coefficients in the sense that $\theta(n) / \theta_{0}(n) \rightarrow 0$ as $n \rightarrow \infty$ where $\theta(n)=\sum_{j=1}^{p-1} \theta_{j}(n)$. (Received November 23, 1956.)

\section{M. P. Schützenberger: Some properties of semigroups. I.}

Let $A$ be a semigroup and $X$ and $Y$ two subsets of $A$. Define $X Y^{(-1)}=A-(A-X)$ : $Y=\{a \in A: a Y \cap X \neq \phi\} ; \quad Y^{(-1)} X=A-(A-X):: Y ; \quad X / Y=\{(a, b) \in(A \times A):$ $a Y b \subset X\} ; a \equiv b(X)$, if and only if $X / a=X / b$ (cf. M. Teissier, Compt. Rend. vol. 232 (1951) p. 1987); $\psi_{X}$ is the homomorphism attached to $\equiv(X)$. With groups, $\bar{x}(X)$ is the equivalence associated with the largest normal subgroup contained in $X$. In any case, if $\bar{X}=\psi_{X} X, \psi_{\bar{X}}$ is the identical application of $\psi_{X} A$ onto itself. Let $P$ be a subsemigroup of $A$; let the following conditions be defined: $\mathcal{U}: P^{(-1)} P \cap P P^{(-1)}=P ; \mathcal{U}_{l}$ : $P^{(-1)} P=P ; \Re: A^{(-1)} P A^{(-1)}=A ; \Re T_{r}: P A^{(-1)}=A ; Q: P^{(-1)} P P^{(-1)}=A ; Q_{r}: P P^{(-1)}=A$. $\mathcal{U}_{l}\left(\mathscr{U}_{r}\right)$ is the definition of a semisubgroup "unitaire" ("net"), cf. P. Dubreil in Mem. Acad. Sci. vol. 63 (1941) p. 8. Any of these conditions is satisfied simultaneously by $P$ in $A$ and $\psi_{P} P$ in $\psi_{P} A$. (Received November 5,1956 .)

\section{7t. M. P. Schützenberger: Some properties of semigroups. II.}

1. If $A$ is free, the subsemigroups satisfying $\mathcal{U}$ form the complete lattice of the free subsemigroups of $A$. From this a new simple proof of the corresponding Nielsen Schreier theorem for groups can be obtained by using an adequate $\psi_{Q}$. From now on we assume that $\psi_{P} A$ satisfies the descending chain condition for left and right ideals. 2 . If $\mathcal{U}$ and $\Re_{r}$, then $\mathcal{U}_{l}$; if $\mathcal{U}_{\text {and }} \mathcal{U}_{l}$, then $\mathfrak{T}_{r}$; if $\mathcal{U}_{l}$ and $Q$ then $Q_{r}$. 3. If $\mathcal{U}$ and $\Re$, then $Q$ if and only if the Suschkevitsch group of $\psi_{P} A$ (cf. A. H. Clifford, Amer. J. Math. vol. $70(1948)$ p. 520) is reduced to its unit element. The proofs are based on the representation indicated in Compt. Rend. vol. 432 (1956) p. 2907. The results, although they are similar, do not coincide with those given in Trans. IRE, vol. IT-2, 47-80 (Sept. 1956), where an interpretation of $Q_{r}(Q)$ as a condition of ergodic indecomposability for (generalized) recurrent events is discussed in detail. (Received November 5, 1956.)

\section{8t. B. M. Stewart: Sums of powers of digits.}

Let $A=\sum a_{i} B^{i}$ be a positive integer expressed to the base $B$. Define $G(A)=\sum a_{i}^{t}$ where $t$ is a fixed positive integer. There exists $A^{\prime}$ such that $G\left(A^{\prime}\right) \geqq A^{\prime}$, but $G(A)<A$ for all $A>A^{\prime}$. When $t=1, A^{\prime}=B-1$ and when $B=2, A^{\prime}=1$ trivially. In the nontrivial cases, $A^{\prime}<K=(t-1) B^{t}$. When $B \geqq t^{2}, A^{\prime}=K-1$. In other cases an algorithm is given for determining $A^{\prime}$. By the iterative notion of Isaacs (Canadian Journal of Mathematics vol. 2 (1950) pp. 409-416) $G(A)$ divides the positive integers into $T$ classes. $T$ is finite since $T \leqq A^{\prime}$. Further observations about $T$ are given. The case $B=10, t=2, A^{\prime}=99, T=2$ was discussed by Porges (Amer. Math. Monthly vol. 52 (1945) pp. 379-382). (Received November 13, 1956.)

\section{9t. M. H. Stone: Some algebraic aspects of logic.}

Using a more or less standard formalization of the lower predicate calculus in terms of finite symbol-sequences, this paper shows that in terms of logical equivalence this system is to be identified as a free, locally finite, polyadic Boolean algebra in the 
sense of Halmos. The principal result of the paper includes as a special case the statement that any locally finite polyadic Boolean algebra is homomorphic to one of these free algebras obtained by a suitable specification of its basic symbols. This special case is the justification for our use of the term "free." In its general form the principal result serves to unify and simplify a series or extension and embedding theorems which occupy a central place in Halmos's theory of polyadic Boolean algebras. Work on this paper was sponsored by the United States Air Force, Office of Scientific Research, Division of Mathematics, under Contract No. AF 18(600)-1125. The paper is to be distributed as a Technical Report under this contract. (Received October 8, 1956.)

190. T. T. Tanimoto: A theorem concerning doubly stochastic matrices.

Let $\mathfrak{M}_{n}$ be the set of all $n \times n$ real matrices, and let $\Gamma_{n}$ be a linear operator on $\mathfrak{M}_{n}$ into the reals such that if $A=\left(a_{i j}\right) \in \mathfrak{M}_{n}$, then $\Gamma_{n}(A)$ is the sum of $n$ elements of $A$, no two in the same row of column. $\bar{\Gamma}_{n}$ is said to be maximal (minimal) with respect to $A$ if $\bar{\Gamma}_{n}$ is a maximum (minimum) over all possible $\Gamma_{n}$ 's. If $c$, called the global constant of $A$, is the sum of all the elements of $A$, then there exists a unique doubly stochastic matrix $S=\left(s_{i j}\right) \in \mathfrak{M}_{n}$ defined by $s_{i j}=\left(k+b_{i j}\right) / k n+c$, where $b_{i j}=\sum_{i} a_{i j}$ $+\sum_{i} a_{i j}-n a_{i j}$ and $k=0$ if $\min _{i j} b_{i j} \geqq 0$, and $k=-\left(\min _{i j} b_{i j}\right)$ if $\min _{i j} b_{i j}<0$, such that if $\bar{\Gamma}_{n}$ is maximal with respect to $A$, then $\bar{\Gamma}_{n}$ is minimal with respect to $S$. Conversely, given any doubly stochastic matrix $S \in \mathfrak{M}_{n}$ and a constant $c$, if $n>2$, there exists a unique matrix $A \in \mathfrak{M}_{n}$ having $c$ as its global constant such that if $\bar{\Gamma}_{n}$ is minimal with respect to $S$, then $\bar{\Gamma}_{n}$ is maximal with respect to $A$. The proof depends upon the solution of a linear system of $n^{2}$ equations in $n^{2}$ unknowns whose matrix $D$ of the coefficients in terms of direct products is $D=R \times I+I \times(\Xi-I)$, where $R \in \mathfrak{M}_{n}$ is the matrix consisting of ones except for the main diagonal whose elements are all $2-n, \Xi \in \mathfrak{M}_{n}$ is the matrix consisting entirely of ones, and $I \in \mathfrak{M}_{n}$ is the identity matrix. When $n>2, D$ is never singular. (Received November 13,1956.)

\section{E. T. Wong: On injective rings.}

Let $R$ be a left faithful ring i.e., $x \in R, x \neq 0$ then $x R \neq 0$. Consider the additive group $R^{+}$of $R$ as a right $R$-module. $M$ is any right $R$-injective module, then $\bar{M}=M / H$, where $H$ is a maximal submodule of $M$ which $H \cap R^{+}=0$, is a right $R$-injective module and is the maximal essential extension of $R^{+}$. In the ring $Q=\operatorname{Hom}_{R}(\bar{M}, \bar{M})$, if $a \in Q, a \neq 0$ then $a R^{+} \neq 0, Q$ is a self right injective ring containing $R$. Also $Q$ is the unique maximal right quotient ring of $R$. If $S$ is a self right injective ring containing $R$ and $s R=0$ if $s=0, s \in S$, then $S \supset Q . Q$ is a regular ring if and only if the singular ideal of $R$ is zero. (Received November 13, 1956.)

\section{ANALYsis}

\section{D. G. Aronson: The fundamental solution of a linear parabolic} equation containing a small parameter. Preliminary report.

Consider the differential equation $\left(^{*}\right) L_{\epsilon}(u)=\epsilon \sum_{i, j-1}^{n} a_{i j}(x, y) \partial^{2} u / \partial x_{i} \partial x_{j}$ $+\sum_{i=1}^{n} a_{i}(x, y) \partial u / \partial x_{i}+a(x, y) u-\partial u / \partial y=0$, where $\epsilon>0$ is a parameter, and the matrix $\left\|a_{i j}(x, y)\right\|$ is positive definite and symmetric for all $x=\left(x_{1}, \cdots, x_{n}\right)$ and $y^{\prime} \leqq y \leqq y^{\prime \prime}$. Under suitable regularity conditions on the coefficients of $\left(^{*}\right)$, it is shown that the fundamental solution $\Gamma(x, y ; \xi, \eta ; \epsilon)$ of $\left({ }^{*}\right)$ can be written in the form ${ }^{(*)} \Gamma(x, y ; \xi, \eta ; \epsilon)=z(x, y ; \xi, \eta ; \epsilon)+\int_{\eta}^{y} \int z(x, y ; s, \tau ; \epsilon) g(x, \tau ; \xi, \eta ; \epsilon) d s d \tau$, where the parametrix $z$ is given explicitly and $g$ is the solution of a certain integral equation. 
The parametrix employed differs from those of Dressel (Duke Math. J. vol. 7, pp. 186-203; vol. 13, pp. 61-70) and Feller (Math. Ann. vol. 113, pp. 113-160) in that it essentially takes into account the full dependence of $\Gamma$ on $\epsilon$. Moreover, it is shown that for suitable $\psi, \lim _{\epsilon \rightarrow 0+} \int \psi(\xi) \Gamma(x, y ; \xi, \eta ; \epsilon) d \xi=\lim _{\epsilon \rightarrow 0+} \int \psi(\xi) z(x, y ; \xi, \eta ; \epsilon) d \xi$ $=\Psi(x, y ; \eta)$, where $\Psi$ is the solution of the reduced equation $L_{0}(u)=0$ such that $\Psi(x, \eta ; \eta)=\psi(x)$. The proof of the representation (**) follows closely the corresponding proof given by Dressel (loc. cit.). (Received November 13, 1956.)

\section{Nachman Aronszajn: On a problem of Hermann Weyl in the theory of singular Sturm-Liouville equations.}

The problem concerns the changes in spectrum or spectral measure corresponding to a Sturm-Liouville equation $-\left(p x^{\prime}\right)^{\prime}+q x=\lambda x, 0 \leqq t \leqq \infty$ in the limit point case when the boundary condition $\sin \alpha x(0)-\cos \alpha p(0) x^{\prime}(0)=0$ is being changed. The main result is as follows: if $\rho_{\alpha}$ and $\rho_{\beta}$ are corresponding spectral functions with $\alpha \neq \beta$ and $\rho_{\alpha}=\rho_{\alpha}^{0}+\rho_{\alpha}^{\prime}, \rho_{\beta}=\rho_{\beta}^{0}+\rho_{\beta}^{\prime}$ are the decompositions of the measures into absolutely continuous and singular parts, then the measures $d \rho_{\alpha}^{0}$ and $d \rho_{\beta}^{0}$ are equivalent, whereas $d_{\rho_{\alpha}}^{\prime}$ and $d_{\rho} \rho_{\beta}^{\prime}$ are orthogonal. By using results of Gelfand-Levitan, (Izvestiya Akad. Nauk. USSR Ser. Math. 16 (1952)), two examples of Sturm Liouville equations are constructed such that (1) for one boundary condition the spectrum is pure continuous, whereas for all other boundary conditions it is a pure point spectrum composed of isolated eigenvalues; (2) for one boundary condition the spectrum is a pure point spectrum with eigenvalues dense on the real axis and for all other boundary conditions it is a pure continuous spectrum. (Received November 13,1956.)

194. Nachman Aronszajn and W. F. Donoghue (p): On the exponential representation of analytic functions with positive imaginary part in the upper half-plane.

It is well-known that such functions $\phi(\zeta)$ have canonical representations $\phi(\zeta)$ $=\alpha \zeta+\beta+\int\left[(\lambda-\zeta)^{-1}-\lambda\left(\lambda^{2}+1\right)^{-1}\right] d \mu(\lambda)=\exp \left[\sigma+\int\left[(x-\zeta)^{-1}-x\left(x^{2}+1\right)^{-1}\right] f(x) d x\right]$ where $\alpha \geqq 0, \beta$ real, $d \mu$ is a positive measure satisfying $\int\left(\lambda^{2}+1\right)^{-1} d \mu<\infty, \sigma$ real and $0 \leqq f(x) \leqq 1$ for $-\infty<x<\infty$. The first representation can be simplified to the form $\phi(\zeta)=\alpha \zeta+\beta_{\infty}+\int(\lambda-\zeta)^{-1} d \mu$ if and only if $\int|\lambda|\left(\lambda^{2}+1\right)^{-1} d \mu<\infty$. Our purpose is to give relations between the behavior of $\mu$ and $f$ at $\infty$. For an integer $h \geqq 0$ we define $\mathfrak{M}_{h}=\left[\mu: \int|\lambda|^{h}\left(\lambda^{2}+1\right)^{-1} d \mu<\infty\right], \xi_{h}=\left[g(x): \int|x|^{h}\left(x^{2}+1\right)^{-1}|g(x)| d x<\infty\right]$. For a function $f(x)$ with $0 \leqq f(x) \leqq 1$ we define $f_{1}(x)=f(x)$ for $x \geqq 0, f(x)-1$ for $x<0: f_{2}(x)=f(x)-1$ for $x>0,=f(x)$ for $x<0 ; f_{3}(x)=f(x)-1$. Our results are the following: (I) for $h>0: \alpha>0$ and $\mu \in \mathbb{M}_{h}$ is equivalent to $f_{1} \in \mathfrak{G}_{h+1}$; (II) for $h \geqq 1: \alpha=0, \mu \in \mathfrak{M}_{h}$ and $\beta_{\infty}>0$, is equivalent to $f \in \mathbb{S}_{h}$; (III) for $h \geqq 1: \alpha=0, \mu \in \mathfrak{M}_{h}$ and $\beta_{\infty}<0$ is equivalent to $f_{3} \in \mathbb{S}_{h} ;$ (IV) for $h \geqq 2: \alpha=0, \mu \in \mathfrak{M}_{h}$ and $\beta_{\infty}=0$ is equivalent to $f_{2} \in \mathbb{G}_{h-1}$. (The representations II and III for $h=2$ coincide with a theorem of Verblunsky and Delange.) (Received November 13, 1956.)

\section{M. G. Arsove: Composite functions and mass distributions.}

Let $w=u-v$, where $u$ and $v$ are locally bounded subharmonic functions on a region $\Omega$, and let $\phi$ be a $C^{\prime \prime}$ function on a closed interval containing the range of $w$. Then the composite function $\phi \circ w$ is almost $\delta$-subharmonic on $\Omega$, and its mass distribution $\mu$ is given in terms of the mass distribution $m$ for $w$ as $\mu(E)=\int_{E}\left(\phi^{\prime} \circ w\right) d m$ $-(1 / 2 \pi) \int_{E}\left(w_{x}^{2}+w_{y}^{2}\right) \cdot\left(\phi^{\prime \prime} \circ w\right) d a, E$ being any bounded Borel set with closure in $\Omega$. In particular, absolute continuity of $m$ implies absolute continuity of $\mu$. (The case of $w$ 
a continuous subharmonic function and $\phi=\exp$ has been established by M. O. Reade). (Received November 13, 1956.)

196. J. H. Barrett: Complex differential equations with respect to a real variable.

Solutions of the second-order differential equation (1) $\left(y^{\prime} / q\right)^{\prime}+\bar{q} y=0$ where $q=q_{1}(x)+i q_{2}(x) \neq 0$ and $q_{1}(x)$ and $q_{2}(x)$ are continuous real functions of the real variable $x$ on $a \leqq x<\infty$, are investigated with emphasis on oscillation and boundedness. Explicit solutions are given for $q=r(x) \exp (i \theta(x))$, where $\theta^{\prime} / r$ is constant (e.g. $q_{1}$ and $q_{2}$ linearly dependent; $\left.q=\exp (i x)\right)$. Recall that for $q$ real $\neq 0, \sin \int_{a}^{x} q$ and $\cos \int_{a}^{x} q$ are linearly independent solutions of (1) which oscillate if and only if $\int_{a}^{\infty}|q|=\infty$. Define $y=c[a, x ; q]$ to be the solution of (1) such that $y(a)=1$ and $y^{\prime}(a)=0$ and $y=s[a, x ; q]$ to be the solution satisfying initial conditions: $y(a)=0$ and $y^{\prime}(a)=q(a)$. Then $|s|^{2}+|c|^{2} \equiv 1$ and other "trigonometric" identities are satisfied. Examples are given where the "sine" function, $s$, is oscillatory but the corresponding "cosine," $c$, has no zeros. Conditions are given to insure oscillation (or nonoscillation) of solutions of (1). Finally, solutions of the more general complex equation (2) $\left(p y^{\prime}\right)^{\prime}+f y=0$ are expressed in terms of solutions of (1) thus effecting a Prüfer-type transformation of (2). Boundedness Theorems for (2) occur as a by-product as in the author's previous work on the real case (Proc. Amer. Math. Soc. vol. 6 (1955) pp. 247-251) and the matrix case (to appear in Proc. Amer. Math. Soc.). (Received November 13, 1956.)

\section{7t. Anatole Beck: On a functional equation in Banach space.}

If $\mathfrak{X}$ is a Banach space and $T$ is a bounded linear operator in $\mathfrak{X}$ with $\|T\| \leqq 1$, then there exist a finite measure space $(S, \Sigma, m)$, an ergodic measure-preserving transformation $h$ in $S$ and a strongly measurable function $X$ from $S$ into $\mathfrak{X}$ for which $T(X(s))=X(h(s))$ almost everywhere in $S$ if and only if there is in $\mathfrak{X}$ an element $x \neq 0$ for which the point-set closure $P=\mathrm{Cl}\left\{T^{i}(x)\right\}_{i=1}^{\infty}$ of the images of $x$ under repeated applications of $T$ has the two properties: $1^{\circ} x \in P$ and $2^{\circ} P$ is compact. (Received November 16, 1956.)

198t. Stefan Bergman: Operators generating solutions of certain differential equations in three variables and their properties. I.

The author constructs solutions of the equation: $\Psi_{x x}+\Psi_{y y}+\Psi_{z z}+\widetilde{F}(y, z) \Psi$ $\equiv \psi_{x x}+\psi_{z Z^{*}}+F\left(Z, Z^{*}\right) \psi \equiv L(\psi)=0$ where $Z=(z+i y) / 2, Z^{*}=(z-i y) / 2$ and $F$ is an entire function of $Z, Z^{*}$. Let $U=Z \zeta+X-Z^{*} \zeta^{-1}, \gamma=1-\int_{0}^{Z} \int_{0}^{Z^{*}} F_{1} \delta_{1}+\int_{0}^{Z} \int_{0}^{Z^{*}} F_{1}$ $\cdot \int_{0}^{Z_{1}} \int_{0}^{Z^{*}} F_{2} \delta_{2}-\cdots, F_{n} \equiv F\left(Z_{n}, Z_{n}^{*}\right), \delta_{n}=\prod_{\mu=1}^{n} d Z_{\mu} d Z_{\mu}^{*}$. Then the functions $\psi^{(N, K)}$ $=(2 \pi i)^{-1} \gamma \int_{C} U^{N} \zeta^{-N+K-1} d \zeta+\sum_{\nu=0}^{k i / 2]} \Gamma^{(N, K, K-2 \nu)} X^{\nu}, K=0,1, \cdots, 2 N, N=0,1, \cdots$, form a set of particular solutions of $L(\psi)=0$. Here $[n / 2]$ is the largest integer which is smaller or equal to $[n / 2] . C$ is a closed curve around $\zeta=0$. Let $P^{(N, K, K-2 \nu)}\left(Z, Z^{*}\right)$ be the coefficients of the development $\left(Z \zeta+X-Z^{*} \zeta^{-1}\right)^{N}$ $\equiv \sum_{K-0}^{2 N}\left[\sum_{\nu-0}^{K-1(k+1) / 2]} P^{(N, K, K-2 \nu)}\left(Z, Z^{*}\right) X^{K-2 \nu}\right] \zeta^{N-K}$. Then $\Gamma^{\left(N, K, K-2^{\nu}\right)}$ are solutions of the equation (A): $N \gamma Z_{Z^{*}} P^{(N-1, K, K)}+\Gamma_{Z Z^{*}}^{(N, K)}+F \Gamma^{(N, K, K)}=0$ and the $\Gamma^{(N, K, K-2 \nu)}$ are solutions of the system (B): $N_{\gamma Z^{*}} P^{N(-1, K, \nu)}-N \gamma_{Z} P^{(N-1, K-2, \nu)}+(N-K-2 \nu+2)$ $\cdot(N-K-2 \nu+1) \cdot \Gamma^{(N, K, \nu+2)}+\Gamma_{z Z^{*}}^{N, K_{*}}+F \Gamma^{(N, K, \nu)}=0, \quad \nu=K-2, \quad K-4, \cdots, K-$ $2[K / 2]$. Further $\Gamma \equiv \Gamma^{(N, K, \nu)}\left(Z, Z^{*}\right)$ are solutions of the form $\Gamma=-\int_{0}^{Z} \int_{0}^{Z^{*}} T_{1} \delta_{1}$ $+\int_{0}^{Z} \int_{0}^{Z^{*}} F_{1} \int_{0}^{Z_{1}} \int_{0}^{Z^{*}}: T_{2} \delta_{2}-\cdots, T_{K} \equiv T\left(Z_{K}, Z_{K}^{*}\right)$, of the equation $T+F \Gamma+\Gamma_{Z Z^{*}}=0$. (Received December 27, 1956.) 
199t. Stefan Bergman: Operators generating solutions of certain differential equations in three variables and their properties. II.

Using the previous results, author defines operators $T_{m}\left(X, Z, Z^{*} ; f\right)$ $=\gamma \sum_{K=0}^{[m / 2]} f^{(m-K)}(Z) X^{m-2 K}\left(-Z^{*}\right)^{K}[(m-2 k) ! k !]^{-1}+\sum_{K=0}^{[m / 2]} X^{m-2 K} \Gamma^{(m-2 K)}\left(Z, Z^{*} ; f\right)$ transforming analytic functions $f \equiv f(\zeta)$ of a complex variable $\zeta$ into solutions $\psi$ of $\boldsymbol{L}(\psi)=0 . \Gamma^{(m \cdot m-2 K)}$ are solutions of a system analogous to (A) and (B), see I. Further real operators $R_{m}\left(X, Z, Z^{*} ; f\right) \equiv\left(T_{m}+\bar{T}_{m}\right) / 2$ are defined transforming analytic functions $f$ into solutions $\Psi$ of $L(\Psi)=0$ which solutions for $X$ real and $Z^{*}=\bar{Z}$ (conjugate to $Z$ ) become real functions. For the $R_{m}$ holds: $R_{m}(X, Z, 0 ; f)=2^{-1}\left[(1 / m !) f^{(m)}(Z) X^{m}\right.$ $\left.+[(m-2 K) ! K !]^{-1} f^{-(m-K)}(0) X^{m-2 K}(-Z)^{K}\right]$. Using the above relation and classical results, it is possible to determine the location and some properties of singularities of $\Psi\left(X, Z, Z^{*}\right)$ from the coefficients $\left\{A_{m n 0}\right\}, m=0,1,2, \cdots, n=0,1,2, \cdots$ of the series development $\sum_{m-0}^{\infty} \sum_{n=0}^{\infty} \sum_{p=0}^{\infty} A_{m n p} X^{m} Z^{n} Z^{* p}$ of $\Psi$ at the origin. Many of these relations are independent of the coefficient $F$ of the equation $L$ or depend only on some properties of $F$. For instance: Let $\left\{A_{m n 0}, n=0,1,2 \cdots\right\}$ for $m=0,1, \cdots, \mathrm{P}$, satisfy the Hadamard conditions, insuring that $f_{m}(Z)=\sum_{n=0}^{\infty} A_{m n 0} Z^{n}$ has $l_{m}$ poles at the points $Z=Z_{m s}$, and let $A_{m n 0} \equiv 0$ for $m>$ P. Then $\Psi$ has as it's only singularities the lines $\left[Z=Z_{m s},|X|<\infty\right], s=0,1, \cdots l_{m-1}, m=0,1, \cdots, P$. (Received December 27, 1956.)

\section{0t. H. D. Block. Discrete isoperimetric-type inequalities.}

The classical isoperimetric inequality bounds the area $A$ enclosed by a curve $C$ in terms of its length $L$. For a gridwork, an analogue of $A$ is $T$, the total number of lattice points inside $C$; an analogue of $L$ is either $N$, the number of boundary lattice points, or $M$, the sum of their multiplicities (the number of missing neighbors). Other analogous quantities are $\bar{T}^{1 / 2}, \bar{N}, \bar{M}$ which are $T^{1 / 2}, N, M$ averaged over rotations of the gridwork. Inequalities relating these quantities are obtained for a gridwork with sufficiently small mesh width $\delta$. Typical results: $|L-\pi \bar{M} \delta / 4| \leqq \pi \delta$, $M^{2} \geqq 16 T, L \geqq \pi \delta\left(\bar{T}^{1 / 2}-1\right)$. (Received November 13,1956 .)

201t. R. P. Boas and R. C. Buck: Notes on polynomials defined by generating relations. I.

Consider the generalized Appell polynomials defined by $A(w) \psi(z g(w))$ $=\sum_{n-0}^{\infty} p_{n}(z) w^{n}$ where $A, \psi$, and $g$ are power series, where $A(0) \neq 0, \psi$ has no zero coefficients, and $g(0)=0, g^{\prime}(0) \neq 0$. If $\psi$ is entire, and the generating series converges in a region containing a zero, $w=a$, of $A$, then it is obvious that there is a non-null representation of zero by the set $\left\{p_{n}(z)\right\}$, namely $0=\sum^{\infty} 0 p_{n}(z) a^{n}$. Further admissible zeros of $A$ contribute further representations of zero. It is shown that the only representations of zero in the form $0=\sum_{n-0}^{\infty} c_{n} p_{n}(z)$ with $c_{n}=O\left(R^{n}\right)$ are linear combinations of representations obtained in this manner from zeros of $A$, provided that $A$ is regular and $g$ is univalent in the disc $|w| \leqq R$. If $g$ is not univalent, there evidently are infinitely many additional representations of zero; for example, if $g\left(w_{1}\right)$ $=g\left(w_{2}\right)$ but $A\left(w_{1}\right) A\left(w_{2}\right) \neq 0$, then $0=\sum p_{n}(z)\left\{\left(w_{1}\right)^{n} / A\left(w_{1}\right)-\left(w_{2}\right)^{n} / A\left(w_{2}\right)\right\}$. This situation arises, for example, with the choice $g(w)=1-e^{w}, \psi(t)=e^{t}$ and $A(w)=1$; when $z$ is chosen as 1 , there result an infinite number of identities relating the exponential numbers considered by G. T. Williams [Amer. Math. Monthly vol. 52 (1945) pp. 323-327] and Touchard [Canadian Journal of Mathematics vol. 8 (1956) pp. 305-320]. (Received October 22, 1956.) 
202t. R. P. Boas and R. C. Buck: Notes on polynomials defined by generating relations. II.

The Jacobi polynomials $P_{n}^{(\alpha, \beta)}(z)$ are generalized Appell polynomials (cf. the preceding abstract) if and only if $\alpha-\beta$ is 0,1 , or -1 [R. C. T. Smith, Proc. Amer. Math. Soc. vol. 7 (1956) pp. 636-641]. However, $P_{n}^{(\alpha, \beta)}(1+z)$ are generalized Appell polynomials [Erdelyi et al., Higher transcendental functions, vol. 3, 1955, p. 264]. The authors use this fact to give a short proof of the known result about the convergence of the Jacobi expansion of a function analytic in an ellipse. (Received October 22, 1956.)

\section{3t. R. P. Boas and R. C. Buck: Notes on polynomials defined by} generating relations. III.

Let $\left\{p_{n}(z)\right\}$ be Humbert's polynomials, defined by $\left(1-3 z w+w^{3}\right)^{-p}=\sum p_{n}(z) w^{n}$. These may be obtained as generalized Appell polynomials, with $g(w)=3 w /\left(1+w^{3}\right)$ [cf. Abstract 202]. The function $g$ is univalent in the disc $|w| \leqq \rho$ for $\rho<(1 / 2)^{1 / 8}$. It is shown that every function which is regular inside the hypotrochoid $3 x=\rho^{-1} \cos \theta$ $+\rho^{2} \cos 2 \theta, 3 y=-\rho^{-1} \sin \theta+\rho^{2} \sin 2 \theta$ has an expansion in Humbert polynomials, convergent in the same region. This is analogous to the elliptical expansion regions for the Gegenbauer polynomials. In particular, if $f$ is regular in a disc $|z|<R$ with $R>(1 / 4)^{1 / 3}$, then its Humbert expansion converges at least in the disc $|z|<r$ where $(R-r)(R+r)^{2}=8 / 27$; for large $R, r=R-O\left(R^{-2}\right)$. Since $g$ is not univalent in the unit disc, there are non-null representations of zero which converge in a region containing the origin and bounded by part of a prolate hypotrochoid. Accordingly, the representation of analytic functions by series of Humbert polynomials is not unique in sufficiently small neighborhoods of the origin. (Received October 22, 1956.)

204t. R. P. Boas and R. C. Buck: Notes on polynomials defined by generating relations. IV.

A class of polynomials can be characterized by the recursion relation $p_{n-1}(z)$ $=\left\{p_{n}(z)-p_{n}(q z)\right\} /(z-q z)$ (suggested to the authors by T. S. Motzkin); they can be shown to be generalized Appell polynomials [cf. the third preceding abstract] with $g(w)=w, A(w)$ arbitrary, and $\psi(t)=\sum_{0}^{\infty}(q-1)^{n} t^{n} /\left\{(q-1)\left(q^{2}-1\right) \cdots\left(q^{n}-1\right)\right\}$. Their formal properties have been investigated independently by Sharma and Chak [Riv. Mat. Univ. Parma vol. 5 (1954) pp. 325-337]. They reduce to classical Appell polynomials when $q \rightarrow 1$. The general expansion theory for generalized Appell polynomials leads, in this particular case, to the following results. When $q>1$, the expansion class is a certain space of entire functions of zero order. When $0<q<1$, its consists of certain functions analytic at the origin. If $A(w)$ is analytic in $|w|<\rho$, then any function analytic in the disc $|z|<\{(1-q) \rho\}^{-1}$ can be expanded in this disc. The second statement depends upon the fact that the sequence $\mu_{n}=(1-q)\left(1-q^{2}\right) \cdots$ $\left(1-q^{n}\right)$ is completely monotonic for $0<q<1$. (Received October 22, 1956.)

205t. R. P. Boas and R. C. Buck: Notes on polynomials defined by generating relations. $\mathrm{V}$.

The Boole polynomials [Jordan, Calculus of finite differences, 2d ed., New York, 1947 , pp. $317 \mathrm{ff}$.] may be shown to be generated by $\left[1+(1+w)^{h}\right]^{-1}(1+w)^{z}$, where the principal values of the powers are to be taken. The formal expansion of a function in a series of Boole polynomials is given by Jordan but its convergence seems not to have been investigated. The authors show that if $0<h \leqq 3$ the expansion of an entire 
function of exponential type has the same convergence properties as its expansion in a series of Newton polynomials [Buck, Trans. Amer. Math. Soc. vol. 64 (1948) pp. 283-298], and if $0<h \leqq 1$ the properties under Mittag-Leffler summation are the same as for the Newton series. In particular, if $0<h \leqq 3$, the Boole expansion converges for functions of exponential type less than $\log 2$. If $h>3$ it converges for functions of exponential type less than $\log [1+2 \sin \pi /(2 h)]$. Similar results hold for the generalized Boole polynomials of Peters [Bull. Amer. Math. Soc. Abstract 62-1-24]. (Received October 22, 1956.)

\section{6t. R. P. Boas and A. C. Schaeffer: Variational methods in entire functions.}

If $f(z)$ is an entire function of exponential type $\tau$ and $|f(x)| \leqq M$ then, according to $\mathrm{S}$. Bernstein, $\left|f^{\prime}(x)\right| \leqq M \tau$. The converse is naturally false. The authors establish some theorems which sharpen the conclusion and do have valid converses. One of these is: If $\tau \leqq \pi$ then $\left({ }^{*}\right)\left|\sum_{k-1}^{n} f^{\prime}(k)\right| \leqq M \pi$ for all integers $n$; if $\tau<\pi$ and $\left(^{*}\right)$ holds there are numbers $f_{0}$ and $K(\tau)$, depending respectively only on $f$ and $\tau$, such that $\left|f(x)-f_{0}\right| \leqq K(\tau) M$. They use these results to answer a question of Gaier's [Proc. Amer. Math. Soc. vol. 6 (1955) p. 879] on the relationship between $\lim n^{-1} \sum_{k=1}^{n} f(k)$ and $\lim x^{-1} \int_{1}^{x} f(t) d t$. When $n$ is odd the constant $\pi$ in $\left(^{*}\right)$ is sharp, but when $n$ is even it is not. The precise bound is determined explicitly in some cases, but in general it depends on certain hyperelliptic integrals. (Received October 25, 1956.)

\section{F. G. Brauer: Eigenfunction expansions for a class of singular boundary value problems.}

Let $L$ and $M$ be linear ordinary differential operators, with $L$ having higher order than $M$. Suppose $L$ and $M$ are self-adjoint with respect to some given boundary conditions on a finite interval $\delta$, and satisfy certain positivity conditions. Let $(u, v)$ denote the usual $L^{2}$ inner product on $\delta$ and define a new inner product $[u, v]=(M u, v)$ for functions satisfying the boundary conditions. Let $H_{\delta}$ be the Hilbert space completion of this set of functions in the new inner product. The boundary value problem $L x$ $=\lambda M x$ with the given boundary conditions has a countable number of eigenfunc. tions which form a complete set in $H_{\delta}$. There exist a Green's function and a spectral matrix for the equation $L x=\lambda M x$ on an infinite interval. Let $H$ be the Hilbert space space completion in the inner product $[u, v]=(M u, v)$ of the set of differentiable functions vanishing outside a finite sub-interval. The Parseval equality and expansion theorem hold for functions in $H$. (Received November 9, 1956.)

208. C. C. Braunschweiger: A geometric construction of the $M$-space conjugate to an L-space.

The following theorem is proved: Let $X$ be an abstract L-space with F-unit e and positive cone C. Define $S=(C-e) \cap(e-C), p(x)=\inf \{a:(1 / a) x \in S, a>0\}$ and $Y=\{a S: a \geqq 0\}$. Then $Y$ is an abstract $M$-space with unit element $e, n o r m p(x)$ and unit sphere $S$ and is linearly isomorphic and isometric to the space $L^{\infty}(\Omega, m)$ conjugate to the concrete representation $L(\Omega, m)$ of $X$. This geometric construction of the conjugate to a given $L$-space is based upon Clarkson's $A$ geometric characterization of $C$-spaces (Annals of Math. vol. 48 (1947) pp. 845-850). The proofs of the theorem and some related results rely heavily upon the classic papers of S. Kakutani (Annals of Math. vol. 42 (1941) pp. 523-537 and 994-1024). The above theorem is slightly generalized to include the case where $X$ has no $F$-unit by using the fact that then $X$ is a direct sum of $L$-spaces, each with an $F$-unit. (Received October $5,1956$. 
209. H. J. Bremermann: Solution of the Dirichlet problem for pseudoconvex domains by plurisubharmonic functions. Preliminary report.

It is well known that to continuous real boundary values prescribed on the boundary of a domain $D$ in the complex number space $C^{n}, n>1$, there does not exist in general a pluriharmonic function in (real part of a holomorphic function) that assumes the boundary values, not even if these are prescribed only on the (real) $n$-dimensional "distinguished boundary surface." This latter boundary value problem is solved by functions of S. Bergman's "extended class." It is shown that the extended class can be replaced by the class of plurisubharmonic functions. This class solves also the Dirichlet problem for continuous values $v(z)$ prescribed on the whole boundary of a totally pseudo-convex domain $D$. The proof of this result consists in determining the envelope of holomorphy of the set $\left\{(z, w)|z \in \bar{D} ;| w \mid<e^{-v(z)}\right.$ for $z \in \dot{D}, w=0$ for $\left.z \in D\right\}$. It is conjectured that in general the plurisubharmonic functions solve the Dirichlet problem for boundary values prescribed on the Silov boundary of a pseudo-convex domain. (Received November 14, 1956.)

\section{F. E. Browder: Mixed boundary-value problems.}

Let $G_{\imath}$ for each $t \geqq 0$ be a domain $E^{n}$ varying smoothly and monotonically with $t$, while for each $t, A(t), B(t)$, and $C(t)$ are strongly elliptic systems of differential operators in the $x$-variables of orders $2 m, 2 r$, and $2 s$ respectively with coefficients defined on $G_{t}(A(t)$ positive). Let $u(x, t)$ be drawn from the class of functions defined for $t>0$ and $x$ in $G_{t}$ and satisfying one of a certain class of null elliptic boundary conditions on the boundary of $G_{t}$ for all $t$. Under certain smoothness and boundedness hypotheses on the coefficients of the operators $A, B$, and $C$, existence, uniqueness, and regularity theorems are established for the solution of each of the following problems: (1) $(1)^{m} A(t)\left(\partial^{2} u / \partial t^{2}\right)+(-1)^{r} B(t)(\partial u / \partial t)+C(t) u=f(x, t)$, with $u$ and $\partial u / \partial t$ prescribed at $t=0 ;(2)(-1)^{m} A(t)(\partial u / \partial t)+(-1)^{r} B(t) u=f(x, t)$ with $u$ prescribed at $t=0$. Regularity properties on the boundary are established, and in particular it is shown that, with smooth data, the solutions and their appropriate derivatives assume their prescribed boundary values. The theorems established extend previous results of Ladizenskaya, Visik, Yosida, and Lions. (Received November 13, 1956.)

\section{Leon Brown: On Erhardt Schmidt branching theory.}

We are interested in generalizing the Schmidt branching theory, and take the functional analysis point of view. Let $X$ be a complex $B$ space and $f(x)$ be any given function with domain and range in $X$, which is analytic and bounded for $\|x\| \leqq 1$. We assume $f(0)=0$ and $f^{\prime}(0)$, a linear function on $X$ to $X$, is a compact transformation. We consider the functional equation $x-f(x)=y$; where $y$ is given. The situation where $\left(I-f^{\prime}(0)\right)^{-1}$ exists is well known. A more interesting problem presents itself when the inverse of $I-f^{\prime}(0)$ does not exist. In this case, we find that there exists: (1) a finite dimsional subspace $M C X,(2)$ a subspace $N C X$, (3) $f_{i}(y, z), i=1, \cdots, k$, analytic in a neighborhood of the origin of $X \times M$ with range contained in the complex numbers, and (4) $x_{1}(y, z)$ analytic in a neighborhood of the origin of $X \times M$ with range contained in $N$. These are related to our problem in the following ways: (1) $x \in X$, then $\left(I-f^{\prime}(0)\right) x=0$ iff $x \in M$, and (2) $x \in X$, then $x-f(x)=y$ iff $x$ $=x_{1}(y, z)$ where $x_{1} \in N$ and $z \in M$, and $f_{i}(y, z)=0, i=1, \cdots, k$. We observe that the set of solutions is contained in a finite dimensional analytic manifold of $X$. The sizes of all the above mentioned neighborhoods are estimated in this paper. (Received November 14, 1956.) 
212t. T. S. Chihara: On co-recursive orthogonal polynomials. Preliminary report.

Let $\left\{P_{n}(x)\right\}$ be the orthogonal polynomials defined by the recursion formula (1) $P_{n}(x)=\left(x+b_{n}\right) P_{n-1}(x)-\lambda_{n} P_{n-2}(x)(n \geqq 2) ; P_{0}(x)=1, P_{1}(x)=x+b_{1}\left(\lambda_{n}>0, b_{n}\right.$ real $)$. The "co-recursive" polynomials $\left\{P_{n}^{*}(x)\right\}$ are defined as the polynomials which satisfy (1) subject to the "initial conditions": $P_{0}^{*}(x)=1, P_{1}^{*}(x)=P_{1}(x)-c\left(c\right.$ real). The $P_{n}^{*}(x)$ are orthogonal polynomials related to the $P_{n}(x)$ by: $P_{n}^{*}(x)=P_{n}(x)-c Q_{n-1}(x)$, where $Q_{n}(x)$ satisfies: $Q_{n}(x)=\left(x+b_{n+1}\right) Q_{n-1}(x)-\lambda_{n+1} Q_{n-2}(x) \quad(n \geqq 1) ; Q_{0}(x)=1, Q_{-1}(x)=0$. The zeros of $P_{n}^{*}(x), P_{n}(x)$ and $Q_{n-1}(x)$ are mutually separated, and various relations among the distribution functions for the three systems of orthogonal polynomials are easily deduced. The $Q_{n}(x)$ have been studied by Shohat and Sherman [Proc. Nat. Acad. Sci. U.S.A. vol. 18 (1920) pp. 282-287] and Sherman [Trans. Amer. Math. Soc. vol. 25(1), pp. 64-87], and some of their methods and results are applied to the study of the $P_{n}^{*}(x)$. The co-recursive polynomials for some special cases give rise to simple examples of orthogonal polynomials whose distributions are of "mixed" type; i.e., the distribution is a step function (with a single jump) on part of the orthogonality interval and a weight function on the remainder of the interval. (Received November $13,1956$.

\section{Joshua Chover (p) and J. Feldman: On positive-definite inte-} gral kernels and a related quadratic form.

Let $C(J)$ be the Banach space of continuous complex functions on an interval $J$, and $B(J)$ the dual space of Radon measures. Let $\rho$ be defined on $J \times J$ and strictly positive-definite. Let $\sigma=\left\{t_{i}\right\}$ be a finite collection of distinct points of $J$, and for any $x \in C(J)$, form $\mu_{\sigma}(x)=\sum_{i, j} r_{i j} x\left(t_{i}\right) x\left(t_{j}\right)$, where $\left[r_{i j}\right]$ is the matrix inverse to $\left[\rho\left(t_{i}, t_{j}\right)\right]$. Theorem: The collection of those $x \in C(J)$ for which $\mu_{\sigma}(x)$ remains bounded under refinements of $\sigma$ is identical with the range of $S^{1 / 2}$, where $S$ is the continuous operator from $L_{2}(J)$ to $C(J)$ defined by $\int_{J} \rho(t, u) h(u) d u$. A corollary: If $J^{\prime}$ is a subinterval of $J$ and $S^{\prime}$ the corresponding integral operator from $L_{2}\left(J^{\prime}\right)$ to $C\left(J^{\prime}\right)$, then range $\left(S^{\prime 1 / 2}\right)$ contains range $\left(S^{1 / 2}\right)$ with functions restricted to $J^{\prime}$. If the operator $R$ from $B(J)$ to $C(J)$ is given by $R m=\int_{J} \rho d m$, one can define $R^{1 / 2}$ from $B(J)$ to $L_{2}(J)$. Theorem: $\left(h, R^{1 / 2} m\right)=\int_{J} S^{1 / 2} h(u) d \overline{m(u)}$ for all $h \in L_{2}(J)$ and $m \in B(J)$; and $S^{1 / 2} R^{1 / 2}=R$, range $(R)$ being a proper subset of range $\left(S^{1 / 2}\right)$. For suitable $\rho, C(J)$ may be considered as a probability space, and $X_{t}=x(t)$ a (Gaussian) stochastic process with $\rho$ as covariance function. Then $\mu_{\sigma}(x)$ may be interpreted as a component of a finite-dimensional approximation to a maximum likelihood ratio, based on "sampling" $x(t)$. (Received November $13,1956$.

\section{Albert Edrei (p) and W. H. J. Fuchs: Deficient values which are also asymptotic.}

The authors prove the following inequality for the Nevanlinna characteristic $T(r)$ of a meromorphic function $f(z)[f(0)=1]$. Let $q$ be a positive integer and let $\sigma \geqq \lambda>1$. Then $T(r) \leqq A \sigma^{q-1} T(r / \sigma)+B \sigma^{-q} T(\sigma r)+C q \sigma^{q-1}\{N(\sigma r, 0)+N(\sigma r, \infty)\}$ where the positive constants $A, B, C$ depend only on $\lambda$ and $N(r, a)$ is the usual counting function of the Nevanlinna theory. If $\lim \sup \{N(r, 0)+N(r, \infty)\} / T(r)$ is small, the above inequality implies that the growth of $T(r)$ is fairly regular. Several applications of this remark are made. In particular it is shown that if $f(z)$ is entire, of finite order $\rho$, and if the sum $\Delta$ of the finite deficient values of $f(z)$ is sufficiently close to 1 , then there are deficient values whose deficiencies exceed $1 / 32 \rho$ and these values are also asymptotic. 
The special case $\Delta=1$ has been investigated by A. Pfluger who proved [Commentarii Mathematici Helvetici vol. 19, pp. 91-104] that the order of $f(z)$ is necessarily an integer $p$ and that all deficiencies are multiples of $1 / p$. The authors now add that all deficient values are asymptotic. (Received November $6,1956$.

\section{M. B. A. Foos: On the values of certain sets of modules.}

Let $a$ be a fixed point in $(0,1)$. Let $G^{\prime}$ be any simply-connected domain in $|w|<1$, such that $G^{\prime}$ contains the origin. Let $G$ be any doubly-connected domain contained in $|w|<1$, disjoint from $G^{\prime}$ and separating $G^{\prime}$ and $a$ from $|w|=1$. Suppose neither $G$ nor $G^{\prime}$ contains the point $w=a$. Let $M^{\prime}$ be the reduced module of $G^{\prime}$, and $M$ the module of $G$ for the class of curves separating the boundaries. Let $b$ be a parameter between 0 and 1 . Then by using the quadratic differential $Q(w) d w^{2}=-(w-b)$ $\cdot\left(w-b^{-1}\right)\left[w^{2}(w-a)\left(w-a^{-1}\right)\right]^{-1} d w^{2}$, and the method of extremal metrics the exact range of values of the pairs $\left(M, M^{\prime}\right)$ is found to be a simply-connected domain in the fourth quadrant of the $M, M^{\prime}$-plane, the boundaries of which are given explicitly. Similarly, we can find the exact range of values of the pair $\left(M^{\prime}, M^{\prime \prime}\right)$, the reduced modules of $G^{\prime}, G^{\prime \prime}$, which are disjoint simply-connected domains containing zero and the point at infinity, respectively, and such that neither contains the point 1. Let $a$ and $b$ be two positive, finite, fixed points. Let $G^{\prime}$ be a simply-connected domain containing $w=0$ and let $G^{\prime \prime}$ be a simply-connected domain disjoint from $G^{\prime}$ and containing the point at infinity. Let $G$ be a doubly-connected domain disjoint from $G^{\prime}$ and $G^{\prime \prime}$ and separating $G^{\prime}$ and $a$ from $G^{\prime \prime}$ and $b$, and such that any curve separating the boundaries of $G$ is homotopic to a curve which separates $G^{\prime}$ and $a$ from $G^{\prime \prime}$ and $b$ and is symmetric with respect to the real axis. Suppose neither $G^{\prime}, G^{\prime \prime}$, nor $G$ contains $a$ or $b$ and let $M^{\prime}, M^{\prime \prime}$ be the reduced modules of $G^{\prime}, G^{\prime \prime}$, respectively, and $M$ the module of $G$ for the class of curves separating the boundaries. Then using quadratic differentials and extremal metrics, we find the exact range of values of the triples $\left(M^{\prime \prime}, M, M^{\prime}\right)$. (Received November $\left.13,1956.\right)$

\section{M. B. A. Foos: On a theorem of Kubo and one of Tsuji.}

By using the method of extremal metrics, considerably shorter proofs can be given for the following theorems. I. (Kubo) Let $D$ and $D_{1}$ be two bounded domains of connectivity $n$, both containing the origin and such that $D_{1}$ contains $D$ and is obtained from $D$ only by a variation of the outer boundary component of $D$, the inner boundary components remaining fixed. Let the outer boundary components of $D$ and $D_{1}$ bound a doubly-connected domain of which the module for the class of curves separating the boundaries is $(1 / 2 \pi) \log M$. Denote by $w=F(z),(F(0)=0)$, the analytic function which maps $D$ conformally onto the unit circle slit along radial segments, such that the outer boundary component of $D$ maps into $|w|=1$. Denote by $w=F_{1}(z),\left(F_{1}(0)\right.$ $=0$ ), the same type of mapping for $D_{1}$. Then $\left|F^{\prime}(0)\right| \geqq M\left|F_{1}^{\prime}(0)\right|$. II. Let $D$ and $D_{1}$ be defined as in I. Let $F(z)$ and $F_{1}(z)$ be the corresponding circular slit mappings. Then $\left|F^{\prime}(0)\right| \geqq M\left|F_{1}^{\prime}(0)\right|$. III. (Tsuji). Let $D$ be a simply-connected domain in the $z$-plane which contains the origin and is contained in $|z|<m$. Let $E$ be a continuum which contains the origin and is contained in $D$, such that a disc of radius $b$ about any point of $E$ is contained in $D$. If $D$ is mapped conformally onto $|w|<1$ by the function $w=w(z), z=z(w)$, with $w(0)=0$, then the image of $E$ is contained in $|w| \leqq t<1$, where $t=t(b / m)$ depends on $b / m$ only. The bound we obtain is better than that given by Tsuji. (Received November 13, 1956.) 
217. Avner Friedman: Classes of solutions of linear nonhyperbolic differential equations.

Denote by $C\left\{M_{n}\right\}\left(M_{n}>0\right)$ the class of all indefinitely differentiable functions $f(x)$ defined in a given $m$-dimensional domain $D$ and possessing the following property: if $x \in D_{1}\left(\overline{D_{1}} \subset D\right),\left|\partial^{n} f(x) / \partial x_{1}^{i_{1}} \cdots \partial x_{m}^{i_{m}}\right| \leqq H_{0} H^{n} M_{n}(n=1,2, \cdots)$, where $H_{0}, H$ depend on $f$ and $D_{1}$. Let $A u(x)=g(x)$ be a linear elliptic system of differential equations of order $s$. We can prove: If $g(x)$ and the coefficients of $A$ belong to $C\left\{M_{n}\right\}$, so do the solutions provided that the $M_{n}$ satisfy: $\left(\begin{array}{c}n-s+2 \\ i\end{array}\right) M_{i} M_{n+2-i} \leqq K n M_{n+1}$ $(i=1, \cdots, n-s+2 ; n=1,2, \cdots ; K>0) . M_{n}=n !$ is the analytic case. A similar result holds for the parabolic equation $\partial u(x, t) / \partial t=L u(x, t)$ where $L$ is an elliptic operator and the coefficients of its essential part are functions of $t$ only. The differentiability properties are considered with respect to $x$. (Received October 29,1956 .)

\section{I. S. Gál: On the continuity and limiting values of functions.}

Let $A$ be an index set and let $J$ be a topology on $A$ such that every noncountable subset $B$ of $A$ has a point of accumulation which belongs to $B$. Let $\phi^{i}=\left\{F_{\alpha}^{i}\right\}(i=1,2)$ be a family of filters $\mathcal{F}_{\alpha}^{i}(\alpha \in A)$ defined on a set $X$. We say that $\phi^{1}$ has property (P) with respect to $\phi^{2}$ and $J$ if for every $F_{\alpha_{0}}^{1} \in \mathcal{F}_{\alpha_{0}}^{1}$ there is a neighborhood $N_{\alpha_{0}}$ of $\alpha_{0}$ such that every $\mathcal{F}_{\alpha}^{2}$ with $\alpha \in N_{\alpha_{0}}$ contains a set $F_{\alpha}^{2} \subseteq F_{\alpha_{0}}^{1}$. Theorem: Suppose that $\phi^{1}$ has property $(\mathrm{P})$ with respect to $\phi^{2}$ and $\mathrm{J}$. Let $f$ be a function from $X \subseteq A$ into a metric space $Y$. Then the set of those points $x \in X$ at which $f$ is continuous with respect to $\mathcal{F}_{x}^{1}$ and discontinuous with respect to $\mathcal{F}_{x}^{2}$ is countable.-A similar result can be proved concerning the set $f[\mathcal{F}]$ of cluster points of $f$ with respect to $\mathcal{F}$. (Received November 13,1956 .)

219. T. M. Gallie, Jr.: Mandelbrojt's inequality and Dirichlet series with complex exponents.

Let $\sum_{n=1}^{\infty} c_{n} \exp \left(-\lambda_{n} s\right)$ converge to $f(s)$ in a disc of radius greater than $\pi \tau$ where $\tau=\lim \sup n /\left|\lambda_{n}\right|<\infty$. Suppose $\lim \inf \left(\left|\lambda_{n+1}\right|-\left|\lambda_{n}\right|\right)>0$ but arg $\lambda_{n}$ is arbitrary. Mandelbrojt's inequality estimates $\left|c_{n}\right|$ in terms of $\max |f(s)|$ on a disc of radius $\pi \tau$ onto which $f(s)$ is continued analytically "by moving a disc" of radius $\pi \tau$. It is proved in this paper that the disc can be replaced by the generally much smaller conjugate diagram $C$ of $\sum_{n-1}^{\infty}\left(1-z^{2} / \lambda_{n}^{2}\right)$. Applications follow. Every boundary point of the region of convergence of $f(s)$ is a limit point of zeros of its partial sums. If $f(s)$ is bounded in the vertical angles, however small, between any two intersecting lines, then $f(s) \equiv 0$. If $C$ is "put on top of" a boundary point of the region of convergence, then $f(s)$ has a singular point on $C$. (This contains Polya's theorem: For $\lambda_{n}$ positive, every segment of length $2 \pi \tau$ of the axis of convergence contains a singular point of $f(s)$.) If $\tau=0$, the region of existence of $f(s)$ is the convex region of convergence. (This fact, undoubtedly known to P6lya, contains the Fabry gap theorem for Taylor series.) (Received November 13, 1956.)

\section{R. P. Goblirsch: An area for simple surfaces.}

Let $\Sigma$ be the boundary of a tetrahedron and let $h$ be a homeomorphism of $\Sigma$ into Euclidean 3-space, $E^{3}$. Let $A(h)=\inf _{P} \lim \inf _{n} A\left(h_{n}\right)$ where the infimum is taken over all sequences $P=\left[h_{n}\right]$ of piecewise linear homeomorphisms of $\Sigma$ into $E^{3}$ which converge uniformly to $h$ and where $A\left(h_{n}\right)$ is the elementary area of $h_{n}$. Then, under a certain restriction on $h(\Sigma)$, there is a positive number $k$, independent of $h$, such that $A(h)$ $\leqq k \cdot L(h)$ where $L(h)$ is the Lebesgue area of $h$. The techniques of proof are modelled 
after those used by R. H. Bing (see Bull. Amer. Math. Soc. Abstract 61-6-824) in establishing the existence of a sequence $P$. (Received November 13,1956.)

221. Michael Golomb: The Dirichlet-initial value problem for partial differential equations in several time-like variables.

Let $\Omega_{x}$ be an open set of $R^{n}$ with boundary $\Gamma_{x}, \Omega_{t}$ the quadrant $t_{1}>0, t_{2}>0$ in $R^{2}$, $D_{x}$ a linear differential operator on $\Omega_{x}$ and $D_{t}=a_{12} \partial^{2} / \partial t_{1} \partial t_{2}+a_{1} \partial / \partial t_{1}+a_{2} \partial / \partial t_{2}+a_{0}$, $a_{i} \in L^{\infty}(\Omega), a_{12}(x) \geqq a>0$ for almost all $x \in \Omega$. Given the functions $f, g, g_{i}$ on $\Omega_{x} \times \Omega_{t}$, a solution $u$ of the equation $\left(D_{t}+D_{x}\right) u=f$ in $\Omega_{x} \times \Omega_{t}$ is to be found subject to the conditions $u=g$ on $\Gamma_{x} \times \Omega_{t}$ and $u=g_{i}$ on that part of the boundary of $\Omega_{x} \times \Omega_{t}$ where $t_{i}=0$. That this is a correctly set problem is proved for the case where $D_{x}$ is a general elliptic operator, say of second order. Let $D$ be the space of (Schwartz) testing functions on $\Omega$ and let $\mathcal{E}$ be the Hilbert space defined by functions $u, v$ which together with their first derivatives are in $L^{2}(\Omega)$, the scalar product being defined as $(u, v)_{1}=(u, v)$ $+\sum\left(u_{x_{r}}, v_{x_{r}}\right),(u, v)$ being the usual scalar product in $L^{2}(\Omega)$. Let $D_{1}$ be the closure of $D$ in $\mathcal{E}, D_{1}^{\prime}$ its strong dual, $D^{\prime}\left(\Omega_{t}, D_{1}\right)$ the space of distributions on $\Omega_{t}$ with values in $D_{1}$. Then $D_{t}+D_{x}$ maps $\mathscr{D}^{\prime}\left(\Omega_{t}, D_{1}\right)$ into $\mathscr{D}^{\prime}\left(\Omega_{t}, D_{1}^{\prime}\right)$. The result is that this is an isomorphism onto. (Received December 17, 1956.)

\section{A. W. Goodman: Variation of the branch points for an analytic function.}

Let $w=f(z)$ be regular in $|z|<1$, and map that domain onto a Riemann surface $R$ having at least one simple branch point $B$, and let $f(0)=0, f^{\prime}(0)>0$. Let $R^{*}$ be the new Riemann surface formed from $R$ by moving the branch point $B$ to $B^{*}=B+\lambda$, where $\lambda$ is small, leaving all other branch points fixed. If $f^{*}(z)$ is the function mapping $|z|<1$ onto $R^{*}$ taking $z=0$ into the same place on $R^{*}$ that $f(z)$ does, and having $f^{* \prime}(0)>0$, then $f^{*}(z)=f\left(z^{\prime}\right)+\lambda P(z)+\bar{\lambda} Q(z)+O\left(\lambda^{2}\right)$. The functions $P(z)$ and $Q(z)$ are determined explicitly. Generalizations and applications are discussed. (Received November 8, 1956.)

\section{3t. Donald Greenspan: On a "best" 9-point difference equation} analogue of Laplace's equation.

It is shown that the well known "9-point" difference equation: $-20 u_{0}+4\left(u_{1}+u_{2}\right.$ $\left.+u_{3}+u_{4}\right)+u_{5}+u_{6}+u_{7}+u_{8}=0$, is a best "9-point" approximation for the Laplace equation by showing that there exist no higher order approximations and that there exist no other approximations of the same order. (Received November 1, 1956.)

\section{D. S. Greenstein: Derivative manifolds in $L^{2}$ and the Hamburger} moment problem.

Given $f(x) \in C^{\infty}(-\infty, \infty)$ such that $f^{(n)}(x) \in L^{2}(-\infty, \infty)(n \geqq 0)$, it can be shown that the closed linear manifold $D_{f}$ spanned by $f(x), f^{\prime}(x), f^{\prime \prime}(x), \cdots$ is contained in the closed linear manifold $T_{f}$ spanned by the translates $f(x+h)$. It is of interest to determine when $D_{f}=T_{f}$. This is so if and only if the absolutely continuous mass distribution whose derivative is almost everywhere equal to the square of the modulus of the Fourier transform of $f(x)$ is uniquely determined by its moments. It is further shown that if $D_{f}=T_{f}$, then $D_{f}(n)=T_{f}(n \geqq 0)$ which implies, via the Hamburger moment problem, a new result on $L^{2}$ completeness of polynomials. (Received November $13,1956$. 


\section{Kenneth Hoffman: Boundary behavior of generalized analytic} functions.

Recently, Arens and Singer (Generalized analytic functions, Trans. Amer. Math. Soc. vol. 81 (1956) pp. 379-393) have studied a generalization of part of the theory of analytic functions in the unit disc, stemming from summable functions on partially ordered, locally compact groups. The paper treats of the generalization in this context. Let $G$ be a discretely topologized subgroup of the additive group of real numbers, and let $A$ be the Banach algebra of those summable functions on $G$ which vanish on the negative portion of $G$. The Gelfand representation defines an isomorphism of $A$ with an algebra of continuous functions on the space $\Delta$, of maximal ideals of $A$. The Silov boundary of $\Delta$ is the character group $\Gamma$ of $G$. A function on $\Delta$ is called "analytic" if it can be uniformly approximated on compact subsets of $\Delta-\Gamma$ by representing functions of elements of $A$. For these abstract analytic functions, generalizations are established of Fatou's theorem on bounded analytic functions, Riesz's theorem on functions of Hardy's class $H_{p}$, Fatou's general Dirichlet principle, and Priwaloff's theorem on the measure of the set of boundary zeros of an analytic function. (Received November $13,1956$.

\section{J. A. Hummel: Doubly orthogonal functions.}

Given a bounded linear mapping $J$ of the Hilbert space $H_{2}$ into the Hilbert space $H_{1}$, a set $\left\{\phi_{n}\right\}$ in $H_{2}$ is called doubly orthogonal with respect to $J$ if $\left\{\phi_{n}\right\}$ is complete and orthonormal in $H_{2}$ and $\left\{J_{\phi_{n}}\right\}$ is orthogonal in $H_{1}$ (cf., Bergman, The kernel function, Mathematical Surveys, no. 5, 1950). It is easily shown that the bounded, positive, self-adjoint transformation $A$ defined by $(J f, J g)_{1}=(A f, g)_{2}$ has the property that $\phi \in H_{2}$ is one of a doubly orthogonal set if and only if $\phi$ is a characteristic element of $A$. From this, it is easily shown that a doubly orthogonal set exists whenever $J$ is completely continuous. This result can be applied to a great variety of examples. (Received November 13, 1956.)

\section{R. C. James: Characterizations of reflexivity.}

A reflexive Banach space has the property that each linear functional attains its sup on the unit sphere. The converse of this is true for separable spaces, while a nonseparable Banach space is reflexive if each linear functional attains its sup on the unit sphere of each separable subspace [Bull. Amer. Math. Soc. 62-4-496]. The following theorems are valid for Banach spaces $B$ in general. If $B$ has a complemented, nonreflexive, separable subspace, then there is a linear functional which does not attain its sup on the unit sphere of $B$. If $B$ has an unconditional basis (not necessarily denumerable), then $B$ is reflexive if each linear functional attains its sup on the unit sphere. If $S$ is the unit sphere of $B$, then each of the following is necessary and suffcient for $B$ to be reflexive: If $W$ is a bounded closed convex subset of $B$ with $W \cap S$ $=0$, then $W$ and $S$ can be separated by a hyperplane which does not intersect $W$; if $W$ is a bounded closed convex subset of $B$, then $S+W$ is closed; if $W$ is a bounded closed convex subset of $B$, then the convex hull of $S \cup W$ is closed. (Received November 13, 1956.)

228. W. B. Jurkat: On the converse of Borel's limit theorem by complex methods.

The paper contains a direct proof of the well known statement that (i) $f(z)$ $=e^{-z} \sum s_{n} s^{n} / n !=O(1)$ as $z \rightarrow \infty,|z|-Q_{z}=O(1)$ implies $s_{n}=o(1)$, if the Tauberian 
condition (ii) $a_{n}=s_{n}-s_{n-1}=O\left(n^{-1 / 2}\right)$ is satisfied. The method of proof consists of evaluating $s_{n}$ by a contour integral which can be estimated by proper choice and subdivision of the path of integration. There are several extensions: In condition (i) the variable $z$ may be restricted to real values. The condition (ii) may be replaced by $\sum k\left|a_{k}\right|^{2}=O\left(n^{1 / 2}\right)$ summing from $n$ to $n+n^{1 / 2}$. The results and methods correspond to those of a previous paper concerning Abel's limit theorem. (Received November 19, 1956.)

\section{S. N. Karp: Relations between diffracting cylinders and their diffraction patterns.}

The problem of diffraction of the plane wave $u^{i n c}\left(r, \theta: \theta_{0}\right) \equiv \exp \left[i k r \cos \left(\theta-\theta_{0}\right)\right]$ by a conducting cylinder $C$ involves the determination of a function $u(r, \theta)$, regular outside $C$ and such that (1) $\Delta u+k^{2} u=0$, (2) $u=0$ on $C$; (3) at infinity; $u \rightarrow u^{i n c}\left(r, \theta: \theta_{0}\right)$ $+r^{-1 / 2} f\left(\theta, \theta_{0}\right) \exp i k r$. The function $f\left(\theta, \theta_{0}\right)$ is the diffraction pattern. We show that if $\operatorname{det}\left\{f\left(\theta_{m}, \theta_{n}\right)\right\}=0$, where $\theta_{1} \cdots \theta_{N}$ are $N$ distinct angles, then the equation of $C$ is $\sum_{1}^{N} A_{m} u^{i n c}\left(r, \theta: \theta_{m}\right)=0$, and conversely. It follows that no determinant of order three can ever vanish. Similar results hold for the Neumann Boundary Condition. It is also shown that if $f\left(\theta, \theta_{0}\right)=F\left(\theta-\theta_{0}\right)$ then $C$ is a circular cylinder. (Received December 28, 1956.)

230t. N. D. Kazarinoff: Asymptotic solution with respect to a parameter of ordinary linear second order differential equations in a domain containing a regular singular point.

The differential equations considered are of the type $\left(^{*}\right): d^{2} u / d z^{2}+\lambda^{2} Q(z, \lambda) u=0$, wherein $Q(z, \lambda)=\sum_{0}^{\infty} q_{j}(z) \lambda^{-i}$ and $z$ is confined to a simply connected domain containing the origin and in which $z q_{0}, z q_{1}$, and $z^{2} q_{j}, j \geqq 2$, are analytic. $Q(z, \lambda)$ is assumed to be analytic in $\lambda$ for $|\lambda|>N$. Ziebur [Thesis, University of Wisconsin, 1950] and Olver [Philos. Trans. Roy. Soc. London, Ser. A vol. 249 (1956) pp. 65-97] have investigated the asymptotic behavior of solutions of classes of equations which are subclasses of those of type $\left(^{*}\right)$. The present investigation, as well as those cited, is based upon methods introduced by Langer [Trans. Amer. Math. Soc. vol. 67 (1949) pp. 461490]. The asymptotic behavior of solutions of $\left(^{*}\right)$ is obtained in a full neighborhood of $z=0$ which may be unbounded provided $Q(z, \lambda)$ is suitable bounded in $z$. (Received November 13, 1956.)

\section{1t. J. H. B. Kemperman: On a class of singular integral equations.}

Let $k(s)$ be defined on $[-\infty,+\infty], k(s) e^{u s}$ in $L^{2}(-\infty,+\infty)$ for $\sigma_{1}<u<\sigma_{2}$. Let $\sigma_{1}<\alpha<\sigma_{2}$, and let $B_{\alpha}$ be the Banach space of measurable functions on $[0,+\infty]$ with norm $\sup \left|e^{-\alpha_{s}} y(s)\right|<\infty$. Results are obtained concerning the integral equation $(\lambda-T) y=f,\left(y, f \in B_{\alpha}\right)$, where $T y=\int^{\infty} k(t-s) y(t) d t$; let $S_{\alpha}$ be the corresponding spectrum. (i) If $\sigma_{1} \leqq \beta<\alpha, f-\sum_{1}^{m} a_{j} e^{c_{j}^{a}} \in B_{\beta}$, then, for each $\epsilon>0, y=\sum_{1}^{m} b_{j} e^{c_{j} s}+\sum_{1}^{n} d_{k} e^{\rho k^{a}}$ $+O^{(\beta+\epsilon) \star}$ with $\phi\left(\rho_{k}\right)=\lambda ;\left(a_{j}, b_{k}, d_{k}=\right.$ polynomials, $c_{j}, \rho_{k}=$ constants $)$. Here, $\phi(u)$ $=\int_{-\infty}^{+\infty} k(s) e^{u s} d s$. This implies a result of C. O. Segerdahl, (Thesis, Uppsala, 1939), concerning a homogeneous random process. (ii) Assume $k(s) \geqq 0$. Let $\lambda, \beta$ be real, $\phi(\beta)<\lambda \leqq \phi(\alpha)$. Then $\lambda \in S_{\alpha} ; \lambda$ is an eigenvalue if and only if $\beta<\alpha$. Related results hold when $\phi^{\prime}(\alpha)=0$. The proof of (ii) uses a generalization to Banach spaces of a result due to R. Jentzsch (J. Math. (1912)) concerning the spectrum of a "positive" operator. (iii) Further results, a.o. exact solutions when $k(t-s)$ is degenerate, either for $t>s$ or for $t<s$. (Received November 13, 1956.) 


\section{2t. W. J. Klimczak: The convergence of Airy series.}

Let $a_{n}(z), n=1,2,3, \cdots$, denote the characteristic functions of the Airy differential operator $A_{z}=d^{2} / d z^{2}+z$ corresponding to the characteristic values $\lambda_{n}, n=1,2$, $3, \ldots$. Bounds for $\left|\lambda_{n}\right|$ and for $\left|a_{n}(z)\right|$ when $z$ is real and nonreal are obtained as $n \rightarrow \infty$, and the following theorem proved: If the series (1) $\sum_{n=1}^{\infty} c_{n} a_{n}(z)$ converges absolutely for any nonreal value of $z$, then it converges absolutely everywhere in the complex plane. It is also shown that the series (1) and the associated series $\sum_{n--^{-}}^{\infty}\left(c_{n} k_{n} / \rho\right) \sin \rho z$, where the $k_{n}$ are suitably defined constants and $\rho=\left(-\lambda_{n}\right)^{1 / 2}$, are absolutely equiconvergent. This is a consequence of a convergent expansion found for $a_{n}(z)$ of the form, $a_{n}(z)=k_{n} P(\rho z) \cos \rho z+k_{n} Q(\rho z) \sin \rho z$, where $P(u)$ and $Q(u)$ are series of polynomials in $u$ with real, rational coefficients. Properties of $a_{n}(z)$ for $z$ real are derived, from which it is proved that the sequence of functions $\left\{a_{n}(x)\right\}$ is orthonormal on the interval $(-\infty, 0)$. (Received November 13,1956.)

\section{G. L. Krabbe: On the spectra of certain Laurent matrices.}

As usual, $l_{p}$ denotes the space of all sequences $c=\left\{c_{n}\right\}$ such that $\sum_{n}\left|c_{n}\right| p<\infty$ $(-\infty<n<\infty)$. Let $a$ be a fixed sequence in $l_{1}$, and let $A$ be the function defined by $A(\theta)=\sum_{n} a_{n} e^{i n \theta}(-\infty<n<\infty)$. If $a * c$ denotes the convolution of sequences (see p. 44 in [L. H. Loomis, An introduction to abstract harmonic analysis, New York, 1953]), then the "Laurent matrix" $\left(a_{n-m}\right)$ represents a linear transformation $c \rightarrow c * a$ of $l_{p}$ into itself, which is denoted $T_{p}$. THEOREM: For any $p$ with $1 \leqq p<\infty$, the spectrum of $T_{p}$ is the image of $[-\pi, \pi]$ by $A$. Let $\widehat{G}$ denote the character-group of a compact abelian group $G$; the theorem is easily extended to the case $a \in L^{1}(\widehat{G})$, if $A$ is the Fourier transform of $a$ [loc. cit., Chapter VII], and if $T_{p}$ is the mapping $c \rightarrow c^{*} a$ of $L^{p}(\widehat{G})$ into itself. (Received December $\left.5,1956.\right)$

\section{H. P. Kramer: A generalized sampling theorem.}

A statement to the effect that a function $f(t)$ can be expanded in a series: (1) $f(t)$ $=\lim _{n \rightarrow \infty} \sum_{r--n}^{n} f(k / 2 w)(\sin 2 \pi w[t-k / 2 w]) /(2 \pi w[t-k / 2 w])$ is known as a sampling theorem. A proof of (1) is given under the hypothesis that $f(t)=\int_{-\omega}^{*} e^{2 \pi_{i} \omega} t d s(\omega)$ where $s(\omega)$ is a complex valued function of bounded variation and has equal jumps at the end points $-w, w$ of the spectral interval. Equation (1) is false if the jumps are not equal. For $\left|\lambda_{k}-k\right|<1 / 4$, equation (1) is generalized to the following formula: $f(t)$ $=\lim _{n \rightarrow \infty} \sum_{k=n}^{n} f\left(\lambda_{k} / 2 w\right) h_{k}(t)$ where the family $\left\{h_{k}\right\}$ depends only on $\left\{\lambda_{k}\right\}$ but not on f. (Received November 15, 1956.)

\section{P. D. Lax: The largest eigenvalue as a convex matrix function.}

Notation: Let $X$ be a square matrix all of whose eigenvalues are real, denote by $\lambda(x)$ the largest eigenvalue of $X$. Theorem: Let $A$ and $B$ be a pair of matrices such that all linear combinations $a A+b B$ of them with real coefficients $a$ and $b$ have real eigenvalues. Then $\lambda(A+B)<\lambda(A)+\lambda(B)$. Proof: Let $D$ denote the domain of determinacy of the point $(0,0,1)$ on the plane $t=0$ for the partial differential equation $u_{t}+A u_{s}$ $+B u_{\nu}=0$. The support function of the convex hull of $D$ is $\lambda(a A+b B)$. The result then follows from the convexity of support functions. For $A$ and $B$ symmetric, the result follows from the maximum property of the largest eigenvalue. The theorem has applications to the study of stability of difference operators. (Received November 14, 1956.) 
236t. Walter Leighton and Zeev Nehari: On the oscillation of the solutions of self-adjoint linear fourth-order differential equations.

A systematic investigation is made of the oscillatory behavior of the solutions of $\left(r y^{\prime \prime}\right)^{\prime \prime}+\left(q y^{\prime}\right)^{\prime}+p y=0(r>0)$. It is found that these equations fall into two distinct classes, typified by $y^{\mathrm{iv}}+p y=0$ and $y^{\mathrm{iv}}-p y=0 \quad(p>0)$ in the simplest case. The first case shows many analogies to the second-order equation $y^{\prime \prime}+p y=0$, while the second case presents a number of novel features. (Received November 14, 1956.)

\section{Karel deLeeuw: $A$ duality theorem for Banach spaces with} compact operator group.

Let $A$ be a Banach space with a compact abelian group $G$ of isometries which is such that for each $x$ in $A$ the map $\sigma \rightarrow T_{\sigma}(x)$ of $G$ into $A$ is continuous. Assume that for each character $\chi$ of $G$ the subspace $\left\{x: T_{v}(x)=\chi(\sigma) x\right.$, all $\sigma$ in $\left.G\right\}$ is finite dimensional. Let $A^{*}$ be the dual space of $A$ and $A^{\#}$ the closed $G$-invariant subspace of $A^{*}$ consisting of all $F$ which are such that the map $\sigma \rightarrow T_{\sigma}^{*}(F)$ of $G$ into $A^{*}$ is continuous. Repeat the process to obtain spaces $\left(A^{*}\right)^{*}$ and $\left(A^{*}\right)^{*}$. THEOREM: $A$ and $\left(A^{*}\right)^{*}$ are canonically isomorphic. More particularly, the canonical map of $A$ into $\left(A^{*}\right)^{*}$ is $1-1$,

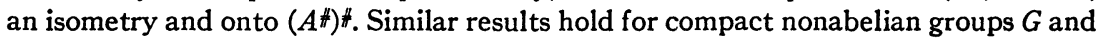
some locally convex spaces $A$. (Received November 14, 1956.)

238. A. J. Lohwater (p) and G. Piranian: Sets of ambiguous points of functions of bounded characteristic.

Let $f(z)$ be a complex-valued function defined in $|z|<1$, and let $\Gamma$ be a simple, closed Jordan curve lying in $|z|<1$ except for one point $P=e^{2 \theta}$. If $f(z)$ approaches two different limits as $z \rightarrow P$ along $\Gamma$ from opposite sides, the point $P$ is called an ambiguous point of $f(z)$. It is known [Bagemihl, Proc. Nat. Acad. Sci. USA vol. 41 (1955) pp. 379-382] that the set of ambiguous points for any complex-valued function is at most denumerable. It is shown that if $E$ is an arbitrary denumerable set on $|z|=1$, there exists in $|z|<1$ an analytic function of bounded characteristic whose set of ambiguous points coincides with $E$. (Received December 27, 1956.)

\section{9t. R. G. Long: A T-system which is not a Bernstein system.}

A fundamental linearly independent sequence $\left\{u_{i}\right\}_{1}^{\infty}$ of points of a real Banach space $E$ is called a $T$-system for $E$ if for each $x$ in $E$ and for each non-negative integer $n, x$ has a unique nearest point, $P_{n}(x)=\sum_{i=1}^{n} x_{i, n} u_{i}$, in $L_{n}=$ lin.ext. $\left[x_{1}, \cdots, x_{n}\right]$. $\left(L_{0}=\{\phi\}\right.$.) The deviation sequence, $\left\{\delta_{i}(x)\right\}_{0}^{\infty}$, of $x$ relative to $\left\{u_{i}\right\}_{1}^{\infty}$ is defined by: (1) $\left|\delta_{i}(x)\right|=\left\|x-P_{i}(x)\right\|$ and (2) for $\delta_{i}(x) \neq 0$, sgn $\delta_{i}(x)=\operatorname{sgn} x_{i^{\prime}, i^{\prime}}$, where $i^{\prime}$ is the least integer greater than $i$ for which $x_{i^{\prime}, i^{\prime}} \neq 0$. If $\left\{\delta_{i}(x)\right\}_{0}^{\infty} \equiv\left\{\delta_{i}(y)\right\}_{0}^{\infty}$ implies that $x=y$, then the $T$-system $\left\{u_{i}\right\}_{1}^{\infty}$ is called a Bernstein system. Kadec [Doklady Akad. Nauk N.S. vol. 92 (1953) pp. 465-468] has shown that any two infinite dimensional Banach spaces with Bernstein systems are homeomorphic and has stated as an open question: Is every $T$-system a Bernstein system? An affirmative answer to this would have shown that all separable infinite dimensional Banach spaces are homeomorphic. This paper exhibits a $T$-system which is not a Bernstein system, using $c_{0}$ with its usual basis and the $T$-norm of Nikol'skir [Doklady Akad. Nauk N.S. vol. 59 (1948) pp. 639-642]. (Received November 13, 1956.)

240. D. B. Lowdenslager: A Poisson formula and an extended class of functions for symmetric bounded complex domains.

Let $D$ be a Hausdorff space, and $B \subset D$. Suppose $G$ is a compact transformation group on $D$, transitive on $B$, and leaving some point $z \in D$ fixed. Let $F$ be a linear 
set of real continuous functions on $D$, containing the constants and invariant under G. If $|f(z)| \leqq \max \{|f(b)|: b \in B\}$ for $f \in F$, then $f(z)=\int_{B} f(b) d m(b)$, where $m$ is the $G$-invariant normalized measure on $B$. A bounded domain in complex $n$-space is called symmetric if for each point $z$ there is an automorphism of the domain with $z$ as sole fixed point. The formula above applies to the real parts of analytic functions on $D$, the closure of the domain, for the nonexceptional domains at least. More generally, it applies to the functions satisfying $\Delta f=0$, where $\Delta$ is an invariant Laplacian on the domain, which is always continuous on $D$. The set $B$ is the distinguished boundary of the domain in either case, and is a proper subset of the topological boundary in general. (Received October 31, 1956.)

\section{Werner Meyer-Koenig: Absolute summability by circle methods.}

Absolute summation by the circle method (or Taylor's method) $T_{\alpha}$ and similar methods $\left(S_{\alpha}, T_{a}^{\prime}\right)$ are studied. These methods prove to be absolutely regular for the same orders for which they are regular. There are immediate consequences, for example (with regard to $\left|S_{\alpha}\right|, 0<\alpha<1$ ): If $f(z)=\sum a_{n} z^{n}$ and $\sum\left|a_{n}\right|<\infty$, then the Taylor series expansion $\sum b_{n}\left(z-z_{0}\right)^{n}$ of $f(1 / z)$ about $z_{0}=1 / \alpha$ is absolutely convergent for $z=1$. Taking into account also $\left|E_{\alpha}\right|$ and $|B|$ there are numerous inclusion relations such as $\left|T_{a}^{\prime}\right| \subset\left|T_{b}^{\prime}\right|,\left|T_{\alpha}\right| \subset\left|S_{\alpha}\right|,|B| \subset\left|S_{\alpha}\right|$ (holding under suitable conditions). Among the results about absolute translativeness the case $\left|T_{\alpha}\right|$ for $\alpha=1 / 2$ (in one direction) is of interest. Since not only $T_{\alpha}$ but each of the above mentioned methods is a circle method in some sense, we shall refer to all of these methods as circle methods. (Received November 15, 1956.)

\section{W. R. Mann: Improved approximations to ordinary and par-} tial differential equations. A comparative study.

L. Fox (Proc. Roy. Soc. A 190 (1947) pp. 31-59) has introduced a method for improving the finite difference approximations to differential boundary value problems. His improvements are achieved by solving a sequence of problems which successively take into account the higher order differences usually neglected when constructing the approximating finite difference equations. An alternative procedure is to make use of the differential equation in constructing an improved difference equation of low order. This second approach not only reduces truncation error, but sometimes brings about important improvement in stability. (Cf. Jim Douglas, Jr., Jour. of Math. and Phys. XXXV (1956) pp. 145-151). This paper is a comparative study of the two methods. The results show that neither is universally superior to the other, but that each has its peculiar advantages which make it much to be preferred in particular cases. (Received November 7, 1956.)

\section{3t. C. B. Morrey, Jr.: On the analyticity of the solutions of ana-} lytic, nonlinear elliptic systems of partial differential equations.

It is proved that if the vector $u^{1}, \cdots, u^{N}$ is a solution of an analytic nonlinear elliptic system of the type considered by Douglis and Nirenberg (Comm. Pure Appl. Math. vol. 8 (1955) p. 532) and if each $u^{j}$ has Hölder continuous derivatives of order $t_{j}$ (in the notation of the cited paper) in a domain $D$, then the $u^{i}$ are analytic in $D$. If the system is strongly elliptic in the sense that the equations of variation are strongly elliptic as defined by Nirenberg (Comm. Pure Appl. Math. vol. 8 (1955) pp. 649-675), the components $u^{i}$ of a solution have continuous derivatives of order $s+s_{i}\left(s=\max s_{j}\right)$ in the neighborhood of and up to an analytic portion $\Gamma$ of the boundary of $D$, and if the derivatives of $u^{i}$ of order $p, 0 \leqq p \leqq s_{j}-1$, are analytic along $\Gamma$, then the $u^{i}$ can 
be continued analytically across $\Gamma$. The proof is much simpler than the proof of the slightly less general theorem of Petrowsky. The proof involves a simple implicit function theorem in Banach spaces (a la Hildebrandt-Graves) and the extension of certain generalized potentials to the complex domain (see E. E. Levi, 1907, E. Hopf, 1932). (Received November 13, 1956.)

\section{4t. T. S. Motzkin and J. L. Walsh: Location of zeros of infra-} polynomials.

If there exists a function $f(z) \equiv\left(z^{n}+\cdots\right) /\left(z^{n}+\cdots\right) \not \equiv 1$ with $|f(z)| \leqq 1$ on a closed set $E, E$ is called a substar of degree $n$. If $E$ is perfect and $F$ a sum of components of the complement of $E$ such that $E+F$ is not a substar of degree $n$, then $z^{n}+\cdots$ with no zero on $F$ is a strong infrapolynomial on $E$. If $E$ is a noncollinear closed set containing the nonempty boundary of its convex hull $H$, then the infra-polynomials on $E$ and $H$ are identical. There exist sets $E$ for which the set of infrapolynomials is not closed. Let $E$ be $0 \geqq y \geqq A(0 \geqq A \geqq-\infty)$ plus $z=i$; a boundedly generated infrapolynomial on $E$ has its zeros in $1 \geqq y \geqq A$ with sum of ordinates of zeros in $1 \geqq y>0$ less than one unless $i$ is the only such zero, and conversely. Let $C_{t}$ be the disc $|z-t \alpha|$ $\leqq t r, 0<a \leqq t \leqq b,|\alpha|>r$; if $E$ lies in $C_{a}$ and $C_{b}$, all zeros $\zeta_{j}$ of an arbitrary infrapolynomial on $E$ lie in $C_{a}, C_{b}$, and some $C_{t}$; if no $C_{t}$ containing $\zeta_{1}$ cuts $C_{a}$ or $C_{b}$, all other $\zeta_{j}$ lie in $C_{a}$ and $C_{b}$. (Received November 20, 1956.)

\section{J. C. C. Nitsche: $A$ bound for the Gaussian curvature of mini-} mal surfaces $z=z(x, y)$.

Let $z=z(x, y)$ be a minimal surface defined over the circle $C\left[\left(x-x_{0}\right)^{2}+\left(y-y_{0}\right)^{2}\right.$ $\left.<R^{2}\right]$. Consider the transformation $\left(^{*}\right) \xi=x+\int_{\left(x_{0}, y_{0}\right)}^{(x, y)} W^{-1}\left[\left(1+p^{2}\right) d x+p q d y\right], \eta$ $=y+\int\left(x_{0}, y_{0}\right) W^{-1}\left[p q d x+\left(1+q^{2}\right) d y\right]$. Here $W=\left(1+p^{2}+q^{2}\right)^{1 / 2} .\left(^{*}\right)$ defines a one-to-one mapping of $C$ onto a domain $\Gamma$ in the $\xi, \eta$-plane conformal with respect to the metrics induced by the minimal surface. $\Gamma$ contains at least the circle $\left(\xi-x_{0}\right)^{2}+\left(\eta-y_{0}\right)^{2}<R^{2}$, and the function $F(\zeta)=(p-i q)(1+W)^{-1}$ is an analytic function of the variable $\zeta=\xi+i \eta$ in $\Gamma$ (see a preceding abstract by the author). For the Gaussian curvature $K=\left(r t-s^{2}\right) \cdot W^{-4}$ of the surface one obtains the expression $K=K(x, y)=-|d F / d \zeta|^{2}$ $\cdot(1+1 / W)^{4}$. As $|F|<1$ in $\Gamma$ one has in $\zeta_{0}=x_{0}+i y_{0}:\left|F^{\prime}\right| \leqq\left[1+((W-1) /(W+1))^{1 / 2}\right] R^{-1}$. Therefore in $\zeta_{0}$ the following inequality holds: $|K| \leqq\left[1+((W-1) /(W+1))^{1 / 2}\right]^{2}$ $\cdot(1+1 / W)^{4} \cdot R^{-2}<20 \cdot R^{-2}$. In particular, if the $x, y$-plane is tangential in $\left(x_{0}, y_{0}\right)$, $|K|<16 \cdot R^{-2}$. For similar inequalities see E. Heinz (Goett. Nachr. Math.-Phys. Kl. IIa, 1952) and E. Hopf (J. Rat. Mech. Anal. vol. 2). (Received December 10, 1956.)

\section{Robert Osserman: On a nonlinear differential inequality.}

The inequality considered is (1) $\Delta u \geqq f(u)$, where $\Delta u$ is the Laplacian in $n$ dimensions. It is proved that if $f(u)$ is a differentiable function with $f(u)>0$ and $f^{\prime}(u)>0$ for all $u$, then necessary and sufficient that there exist a solution of (1) throughout space is that $\int^{\infty}\left(\int f(u) d u\right)^{-1 / 2} d t=\infty$. Somewhat weaker results were proved in 2 dimensions by Wittich (Ganze Lösungen der Differentialgleichung $\Delta u=e^{u}$, Math. Zeit. vol. 49 (1944)) and in 3 dimensions by Haviland ( $A$ note on unrestricted solutions of the differential equation $\Delta u=f(u)$, J. London Math. Soc. vol. 26 (1951)). The proof uses the maximum principle for subharmonic functions, and has several advantages over the method of integral means used by Wittich and Haviland. Namely, it is equally applicable in any number of dimensions, it eliminates the requirement that $f(u)$ be 
convex needed by Wittich and Haviland, and it gives best possible bounds on the largest circle in which a function can satisfy (1), as well as pointwise bounds on a function known to satisfy (1) throughout a given circle. (Received November 9,1956.)

247. C. Y. Pauc: Generalized Jacobians as Radon-Nikodym integrands.

The setting is Cesari's Surface area, Annals of Mathematics Studies vol. 35, Princeton, 1956. The fundamental cell $A$ is a compact polygonal region in $E_{2}$, the mapping $T$ of $A$ into $E_{3}$ of finite area, $\mu$ the Borel measure in $E_{2}$. The cells are the closed polygonal regions in $A$. To each whole Borel set $B_{0}$ of $A$ there corresponds a vector $\phi_{0}=\phi_{0}\left(B_{0}\right)$ whose components are the algebraic area-measures of the $\left(T_{i}, B_{0}\right)$. $J_{0}$ denotes the Radon-Nikodym integrand of $\boldsymbol{\phi}_{0} \mid \mathscr{B}_{0}$ with respect to $\mu_{0}=\mu \mid \mathscr{B}_{0}$ (restriction of $\mu$ to $\left.\mathfrak{B}_{0}\right), J(w)$ the Jacobian vector in $w \in A$. (I) Theorem 1: If there exists a thin net (monotone sequence of cell partitions of $A$ whose boundaries are thin), for which the sequence of the euclidean norms in $E_{2}$ tends to 0 , then $J_{0}=E\left\{J \mid \mathfrak{B}_{0}\right\}$. (II) If $(T, A)$ is an A.C. representation of a surface $S$ of finite area, the cell function $\gamma(I)$ whose components are the algebraic areas of the $\left(T_{i}, I\right)$, can be extended in a unique manner to a measure $\phi$ defined on $B$ and $\phi(B)=\mu$-integral of $J(w)$ over $B$, $\phi_{0}=\phi \mid B_{0}$. If $M$ is any Borel set in $E_{3}, \mathbf{\Omega}(M)=\phi\left(T^{-2}(M \cap[S])\right.$, the Radon-Nikodym theorem applied to $\boldsymbol{\Omega}$ and $\|\boldsymbol{\Omega}\|$ yields a $\mathrm{L}$. C. Young field. Theorem 2: If $F(p, d)$ is a Calculus of Variations integrand, $\mathfrak{B}$ a finite partition of $A$ into Borel sets $B_{1}, \cdots, B_{n}$, $w_{i}$ a point of $B_{i}, i=1, \cdots, n, \sum F\left(T\left(w_{i}\right), \phi\left(B_{i}\right)\right)$ tends to the Cesari-Weierstrass integral of $F$ over $S$ when the norm of $\mathfrak{B}$ tends to 0 . (Received November 5,1956.)

248. L. E. Payne and H. F. Weinberger (p): A generalized Rellich identity.

The authors' upper and lower bounds for solutions of Laplace's equation and quadratic functionals associated with them [J. of Math. and Phys. vol. 33 (1955) pp. 291-307] depend upon an integral identity due to $F$. Rellich. In consequence, they apply only to domains with star-shaped boundaries. Rellich's identity is generalized by applying Gauss's theorem to the identity $\left[\left(a^{k} g^{i j}-a^{i} g^{i k}-a^{i} g^{i k}\right) u, i u, i\right]_{, k}$ $=-2 a^{j} u,{ }_{j} \Delta u+\left(a^{k} g^{i j}-a^{j} g^{i k}-a^{i} g^{i k}\right),{ }_{k} u, i u, j$ where, $i$ denotes covariant differentiation and $\Delta$ the Beltrami operator with respect to a metric $g_{i j}$, while $a^{k}$ is an arbitrary vector field. If, in particular, $a_{i}$ is a unit normal on the boundary, the boundary integral obtained from the left-hand side is the integral of $|\operatorname{grad} u|^{2}-2(\partial u / \partial n)^{2}$, while the right-hand side is easily bounded above and below by multiples of Dirichlet's integral. Thus the bounds given in the above-mentioned paper can be generalized to general boundaries and Beltrami operators. The Rellich identity follows from the above when $g_{i j}$ is Euclidean and $a^{k}=x^{k}$. (Received November 13,1956.)

\section{R. N. Pederson: $A$ unique continuation theorem for solutions of a quasi-linear biharmonic partial differential equation.}

Let $\Delta^{2} u=f\left(x, u, p_{i}, p_{i j}, p_{i j k}\right)$ be defined in a domain $D$ in Euclidean $n$-space, $x \in D\left(\Delta=\sum \partial^{2} / \partial x_{i}^{2}, p_{i}=\partial u / \partial x_{i}\right.$, etc.). We assume the function $f$ to be uniformly Lipschitz continuous in the arguments $u, p_{i}, p_{i j}, p_{i j k}$. The Unique Continuation Theorem for solutions of the above equation is proved by using the following inequality. Let $r=\left(\sum x_{i}^{2}\right)^{1 / 2}$. If $u \in C^{4}$ vanishes near $r=0$ and for $r \geqq R$, then $\int \exp (\alpha / r)$ $\cdot\left(\Delta^{2} u\right)^{2} d V \geqq\left(c / R^{2}\right) \int \exp (\alpha / r)\left|\sum_{i j k} \partial^{3} u / \partial x_{i} \partial x_{j} \partial x_{k}\right|^{2} d V$ for all sufficiently large $\alpha$. The proof of this inequality makes use of an inequality previously proved by the author (see Bull. Amer. Math. Soc. 62-6-699). (Received November 13, 1956.) 


\section{0t. Pasquale Porcelli: On the existence of the Stieltjes-Mean in- tegral.}

All integrals in this paper are Stieltjes-Mean integrals obtained by the finer subdivision process, and all functions considered are bounded. Definition: Let $f$ be a function on $[a, b], g$ nondecreasing on $[a, b]$, and $k>0$, then $M(f, k+) \subset[a, b]$ such that $x \in M(f, k+) \Leftrightarrow x \neq b$ and for each $(x, y)$ there exist points $t$ and $t^{\prime}$ in $(x, y)$ such that $\left|f(t)-f\left(t^{\prime}\right)\right| \geqq k . M(f, k-)$ is defined in a similar manner and $M(f, k)$ is the logical sum $M(f, k+)+M(f, k-)$. $M(f, k)$ is said to have directed $g$-content zero (hereafter denoted by $C_{0} M(f, k)=0$ ) if for each $\epsilon>0$, there exists a finite collection $\left\{\left[a_{p}, b_{p}\right]\right\}$ of nonoverlapping intervals covering $M(f, k)$ such that (i) $\sum\left[g\left(b_{p}\right)\right.$ $\left.-g\left(a_{p}\right)\right]<\epsilon$, (ii) $b_{p} \notin M(f, k+)$, and (iii) $a_{p} \notin M(f, k-)$. Theorem 1. $\int_{a}^{b} f d g$ exists if, and only if, $C_{g} M(f, k)=0$ for every $k>0$. Theorem 2. If $g$ is of bounded variation, then $\int_{a}^{b} f d g$ exists if, and only if, $\int_{a}^{b} f d g^{\prime}$ exists where $g^{\prime}$ is the variation function of $g$. Theorem 3. If $\int_{a}^{b} f d g$ exists, then $\int_{a}^{b}|f| d g$ exists. In the case where $g$ is semi-regular (i.e. $g(x)$ $\neq g(x-)$ if, and only if, $g(x) \neq g(x+)) C_{0} M(f, k)=0$ can be replaced by $1_{g} M(f, k)=0$, where $1_{g} M(f, k)$ denotes the outer $g$-length of $M(f, k)$. (Received November 8,1956 .)

251. Carlo Pucci: Bounds of solutions of boundary problems for elliptic equations. Preliminary report.

Let $A$ be an open bounded set of $R^{m}, f\left(x, u, u_{i}, u_{i j}\right)$ a real function for $x \in \bar{A}, u_{i}$, $u_{i j} \in R(i, j=1,2, \cdots, m)$. Let $f$ and the first derivatives of $f$ with respect to $u$, $u_{i}, u_{i j}$, be continuous in $\bar{A}$ and let $f_{n}^{\prime} \leqq 0, \sum f_{u_{i j}}^{\prime} \lambda_{i} \lambda_{j} \geqq \rho|\lambda|^{2}(\rho>0)$. Bounds are established for the maximum of the absolute value of solutions of the Dirichlet, Neumann and mixed problems for the differential equation $f\left(x, u, u_{i}, u_{i j}\right)=0, x \in A$. In the case of the Neumann problem it is supposed also that $f_{n}^{\prime}<0$ at some points of $A$. The uniqueness of solution is also proved. The results are an extension of Hopf's theorem regarding the Dirichlet problem (Sitzungb. Preuss. Akad. Wiss. vol. 19 (1927) pp. 147-152) and of previous results of the author for linear equations (Rend. Acc. Lincei 8, 13, (1952) pp. 360-366). As a particular case one obtains the following theorem: Let $D$ and $N$ be two sets, $D \cap N=\phi, D \cup N=\dot{A}, D \neq \phi$. The assumption is made that, in every point $x$ of $N, \dot{A}$ has a tangent hyper-plane and a sphere $S$ exists with $x \in S, S \in \bar{A}$. Let $u$ be a function of class $C^{(2)}$ in $A$ satisfying the considered differential equation, continuous in $\bar{A}$ and with normal derivative equal zero on $N$. If $f(x, 0,0,0) \equiv 0$ the maximum (minimum) value of $u$ is assumed on $D$ or is zero. (Received November 14, 1956.)

252t. P. V. Reichelderfer: A study of the essential Jacobian in transformation theory.

Let $T$ be a continuous transformation from a bounded domain $D$ in Euclidean $n$ space $R^{n}$ into a bounded portion of $R^{n}$. For a function of domains $D$ contained in $D$ the concept of a $T$ derivative at a maximal model continuum $C$ for $T$ is introduced, wherein the domains $D$ containing $C$ follow the contour of the transformation as their images $T D$ close down on the point $T C$ in a regular manner. Assume that $T$ is essentially of bounded variation in $D$ and consider the function $\mathfrak{A}_{e}$ of domains whose $\boldsymbol{n}$ dimensional derivative is the essential Jacobian ( $\mathrm{T}$. Rado and P. Reichelderfer, Continuous transformations in analysis, with an introduction to algebraic topology, Die Grundlehren der Mathematischen Wissenschaften vol. 75 (1955) Springer-Verlag). If $C$ is a nonessential maximal model continuum for $T$ then $\mathfrak{A}$, has a $T$ derivative 
at $C$ whose value is zero. If $C$ is an essential maximal model continuum for $T$ not belonging to a certain exceptional set then $\mathfrak{A}_{0}$ has a $T$ derivative at $C$ whose nonzero value is the product of the local topological index for $T$ at $C$ and the local magnification ratio for $T$ at $C$. (Received November 2, 1956.)

253. Walter Rudin: The closed ideals in an algebra of analytic functions.

Let $K$ and $C$ be the closure and boundary, respectively, of the open unit disc $U$. Let $A$ be the Banach algebra whose elements are those continuous functions on $K$ which are analytic in $U$, with norm $\|f\|=\max |f(z)|(z \in K)$. A function of the form $M(z)=B(z) \exp \left\{-\int_{C}(w+z)(w-z) d \lambda(w)\right\}$, where $B$ is a Blaschke product and $\lambda$ is a non-negative measure on $C$, is said to be associated with the subset $E$ of $C$ if all limit points of the zeros of $B$ are in $E$ and if $\lambda$ is concentrated on $E$. Theorem: Suppose $E$ is a closed subset of $C$, of Lebesgue measure zero, $M$ is associated with $E$, and $I(E, M)$ is the set of all $f \in A$ such that $f / M$ is bounded in $U$ and such that $f(z)=0$ on $E$. Then $I(E, M)$ is a closed ideal of $A$, and every closed ideal of $A$ (with the exception of the null ideal) is obtained in this way. Consequences: (1) Every closed ideal of $A$ is principal. (2) $I(E, M)$ is the intersection of maximal ideals of $A$ if and only if $M$ is a Blaschke product without multiple zeros (in particular, $\lambda$ must be zero). (3) $I(E, M)$ is the intersection of primary ideals of $A$ if and only if $\lambda$ is a discrete measure. (Received November 13, 1956.)

254t. Diran Sarafyan: Existence of integral curves for first order ordinary differential equations.

In Cauchy's and Picard's proofs of the existence of a solution to $d y / d x=f(x, y)$ there is, besides the continuity and single-valuedness of $f(x, y)$ another condition. Peano's proof is without this additional restriction but is somewhat involved. This paper is a proof which like Peano makes use of polygonal lines but it is a great deal simpler. Let $m$ and $M$ be the minimum and maximum values of $f(x, y)$ on the closed simply-connected region $R$. From the given point $P_{0}\left(x_{0}, y_{0}\right)$ belonging to $R$ two straight lines with slope $m$ and $M$ are drawn. These lines intersect the vertical through $x$ in $N_{1}$ and $N_{2}$. To a polygonal line $\Gamma_{i}$ there corresponds on the segment $N_{1} N_{2}$ a terminal point $T_{j}$. By Bolzano-Weierstrass Theorem it is known that the bounded infinite set of terminal points $\left\{T_{n}\right\}$ has at least one limit point. The author shows that if $\left\{T_{n}\right\}$ has $j$ limit points then through $P_{0}$ and for the interval $\left[x_{0}, x\right]$ there are $j$ integral curves. (Received November 27, 1956.)

\section{5t. Diran Sarafyan: One-parameter family of integral curves.}

If $f(x, y)$ is a single-valued continuous function of $x$ and $y$ on a simply-connected closed region $R$, then it is known that $d y / d x=f(x, y)$ offers at least one integral curve through any point $P_{0} \in R$. The aim of this paper is to show that from all the integral curves through the region $R$, a one-parameter family of integral curves can be extracted. The approach is three dimensional since $z$ is substituted to $d y / d x$. Then $d y / d x=f(x, y)$ becomes $z=f(x, y)$. An integral curve through $P_{0}$ is projected first on the surface $z=f(x, y)$ and then projected on the $x, z$-plane. Through the use of the series $y=y_{0}+z_{0}\left(x_{1}-x_{0}\right)+z_{1}\left(x_{2}-x_{1}\right)+\cdots+z_{n-1}\left(x-x_{n-1}\right)$ and considering in the $x, z$-plane the area under the projected curve, corresponding to the interval $\left[x_{0}, x\right]$. the author shows $y=\phi(x, k)$ where $k$ is a parameter. (Received November 27,1956 .) 


\section{6t. Diran Sarafyan: The singular points of first order ordinary differential equations.}

The aim of this paper is the determination of the singular points of $d y / d x=f(x, y)$ particularly if the equation is of the variables separable, homogeneous or linear form. A broad criterion of uniqueness for solutions is given: the necessary and sufficient condition under which through any point of a closed simply-connected region $R$, there exists a unique integral curve is that $\exp \int_{a}^{z} \partial f d x / \partial y \neq 0$ where $a$ is an arbitrary constant which in most cases can be taken as equal to zero. The class of differential equations which satisfy this criterion contains as a subclass the equations which satisfy the well known Lipschitz condition. Then, two following theorems are proved. Theorem: The singular points of the differential equations of the variables separable form $d y / d x=h(y) / g(x)$ are represented by the intersection points of the straight lines, $x=\alpha_{n}, n=1,2,3, \cdots ; y=\beta_{n}, m=1,2,3, \cdots$ where $\alpha$ 's and $\beta$ 's are the roots, if any of $g(x)=0$ and $h(y)=0$. Theorem: The integral curves of the homogeneous equation $M d x+N d y=0$ admit singular points whenever $x M+y N=0$ admits finite or infinite number of linear solutions $y=\gamma x$. It is on these lines which are also integral curves that the singular points are located. (Received November 27, 1956.)

\section{Morris Schreiber: Representation of non-normal operators.}

The results reported in [Bull. Amer. Math. Soc. Abstract 62-3-333; see also 61-2-290] are extended as follows (terminology is that of the references). If $A$ is a proper contraction (that is, $\|A\|<1$ ) or a generalized nilpotent, then for any $f \in L_{2}(|z|=1, d \theta)$ the operator $\int_{f} f(z) d F(z)$, where $F$ is the strong operator measure of $A$, is bounded, with norm $\leqq\|f\|_{2}$, so that the map $f \rightarrow \int_{a} f(z) d F(z)$ is a continuous * vector space homomorphism of $L_{2}$ into the uniformly closed ring generated by $A$. Restricted to those $f$ whose Fourier coefficients $c_{n}$ vanish for $n<0$ the map also preserves products. It is also shown that, for fixed $f \in L_{2}$ and $A$ varying over operators of the aforementioned types, the map $\left(A, A^{*}\right) \rightarrow \int_{c} f(z) d F(z)$ is continuous in the uniform, strong, and weak topologies (see Kaplansky, Pacific J. Math. vol. 1 (1951) p. 227). (Received November 14, 1956.)

\section{Edward Silverman: Morrey's representation theorem.}

Morrey showed that a nondegenerate Frechet surface of finite Lebesgue area has a quasi-conformal representation upon the unit circle. Let $p_{i}$ be a dense sequence of points in the point set covered by the surface. Let $x$ be a representation of the surface and define $x_{i}(w)=\left\|p_{i}-x(w)\right\|$. A formulation of quasi-conformality which is valid for surfaces not in Euclidean space and can be used to find a "good" representation of the surface is sup $\left(x_{i u}^{2}+x_{i v}^{2}\right)=\sup \left(x_{i u} x_{k v}-x_{i v} x_{k u}\right)$ a.e. The definition of area is that given in Definitions of Lebesgue area for surfaces in metric spaces, Rivista di Matematica della Universita di Parma vol. 2 (1951). This definition agrees with that of Lebesgue for surfaces in Euclidean space. For a "good" representation, the classical integrand is replaced by the right hand side of the equation given. (Received November $15,1956$.

\section{K. T. Smith: An inequality for the Dirichlet integral.}

If $y=\left(y_{1}, y_{2} \cdots, y_{n}\right)$, let $\tilde{y}=\left(y_{1}, y_{2}, \cdots,-y_{n}\right)$; and let $R_{+}^{n}$ denote the half space $y_{n}>0$. The following inequality for the Dirichlet is proved: there is a constant $c$ such that for $0<\alpha \leqq n, \int_{R^{n}+} \int_{R^{n}}|u(x)-u(y)| 2|x-y|^{\alpha-n}|x-\tilde{y}|^{-\alpha-2} d x d y$ $\leqq(c / \alpha) \int_{R^{n}+} \sum\left|\partial u / \partial x_{k}\right|^{2} d x$. The constant in the inequality has the right order of 
magnitude as $\alpha \rightarrow 0$. In the inequality, $R_{+}^{n}$ can be replaced by any bounded domain $D C^{n} R_{+}^{n}$ whose boundary is of class $C^{(0,1)}$. (Received November 13,1956 .)

260. T. H. Southard: A bivariate analogue of the Aitken-Neville interpolation algorithm. Preliminary report.

A. C. Aitken (Proc. Edinburgh Math. Soc. vol. 3 (1932) pp. 56-76) and E. H. Neville (Journal, Indian Math. Soc. vol. 20 (1933) pp. 87-120) have defined a process for construction of a univariate polynomial interpolant by means of a set of linear interpolations. A bivariate analogue is defined here and its applications are considered. (Received December 6, 1956.)

261t. E. J. Specht and H. T. Jones: The complete continuity of the Neumann-Poincaré integral operator.

Warschawski (Applied Mathematics Series 42, National Bureau of Standards) has introduced a Hilbert space $H$ in connection with the numerical solution of a Neumann-Poincare integral equation. The present paper gives a new proof that the integral occurring in this equation gives rise to a completely continuous operator in $H$. This proof is carried through for multiply connected regions in the plane, uses only the existence of the solution of the exterior Dirichlet problem with constant boundary values and hence generalizes to 3 -space. (Received November 14, 1956.)

\section{W. F. Stinespring: Integration theorems for gages and duality for unimodular groups.}

The author proves analogues of ordinary integration theorems not investigated in the paper of I. E. Segal, $A$ non-commutative extension of abstract integration, Ann. of Math. vol. 57 (1953) pp. 401-457. Among these are a dominated convergence theorem, a Fatou's lemma, a Fubini theorem, and a theorem giving conditions under which a positive central linear functional on a self-adjoint operator algebra is the integral with respect to a gage on the weak closure. Using gage spaces, a global kind of harmonic analysis is developed for unimodular groups. Many of the familiar relations between functions on an abelian group and functions on the character group hold here between functions on a unimodular group $G$ and operators in the "dual gage space" $E$. A new binary operation and involution are defined in $L_{1}(E)$ so that it becomes a Banach algebra with an involution, analogous to $L_{1}$ of the character group under convolution and the corresponding involution in the abelian case. In terms of this *-algebra $L_{1}(E)$, a duality theorem analogous to the Tannaka theorem is proved: there is a 1-1 correspondence between elements of $G$ and nonzero *-homomorphisms of $L_{1}(E)$ into the complex numbers. (Received November 9, 1956.)

\section{3t. C. T. Taam: On the solutions of nonlinear differential equa-} tions. III.

Consider (I): $x^{\prime \prime}+p x+q x^{3}=f$, where $p(t), q(t)$ and $f(t)$ are real-valued, periodic, bounded and Lebesgue-measurable functions with a common least period $L$. It is further assumed that $p(t)$ and $q(t)$ are even functions with positive lower bounds and $f(t)$ an odd function with a non-negative lower bound on $(0, L / 2)$. Several criteria for the existence of periodic solutions, harmonic and subharmonic of order $n$, are obtained and their oscillatory behaviour is described. These criteria are expressed in terms of $L$ and of the bounds of $p, q$ and $f$ and become very simple when $q$ or $f$ are small perturbations. The method of proof is to show that under suitable conditions 
there exists a solution of (I) which vanishes at two given points. (This research was supported by the office of Ordnance Research, U. S. Army, Contract No. DA-36034-ORD-1774.) (Received November 13, 1956.)

\section{S. E. Warschawski: $A$ perturbation method in conformal} mapping.

The mapping function $w=f(z)$ of the interior of a smooth curve $C: z=z(t)$, $0 \leqq t \leqq 1$, onto the circle $|w|<1$ may be obtained as a solution of the LichtensteinGershgorin integral equation. If $\theta(t)=\arg f(z(t))$, then $\theta(t)=\int_{0}^{1} K(t, \tau) \theta(\tau) d \tau+g(t)$ where $K(t, \tau)=\pi^{-1}$ arg $[z(\tau)-z(t)] / \partial t$ and $g(t)$ is known. Suppose $C_{\epsilon}: z=z(t, \epsilon), 0 \leqq t \leqq 1$, $0 \leqq \epsilon \leqq 1$, denotes a family of contours such that for some integer $n, z(t, \epsilon)=\sum_{k=0}^{n} z_{k}(t) \epsilon^{k}$ $+o\left(\epsilon^{n}\right)$ and $z_{t}(t, \epsilon)=\sum_{k=0}^{n} \dot{z}_{k}(t) \epsilon^{k}+o\left(\epsilon^{n}\right)$, where $z_{t}(t, \epsilon)$ and $\dot{z}_{k}(t)$ are Lip $(\alpha)$, for some $\alpha, 0<\alpha \leqq 1$. If $f(z, \epsilon)$ denotes a properly normalized mapping function for $C_{\epsilon}$ and $\theta(t, \epsilon)=\arg f(z(t, \epsilon), \epsilon)$, then there exist functions $\theta_{k}(t), k=0,1, \cdots, n$, such that $\theta(t, \epsilon)=\sum_{k=0}^{n} \theta_{k}(t) \epsilon^{k}+o\left(\epsilon^{n}\right)$ where the $\theta_{k}(t)$ is a solution of $\theta_{k}(t)=\int_{0}^{1} K_{0}(t, \tau) \theta_{k}(\tau) d \tau$ $+g_{k}(t)$. Here $K_{0}(t, \tau)$ is the kernel of $C_{0}$ and $g_{k}(t)$ is known if $\theta_{0}, \cdots, \theta_{k-1}$ are determined. The error term is estimated in terms of the errors in $z(t, \epsilon)$ and $z_{t}(t, \epsilon)$. This result entails: an extension of Hadamard's variation formula for the Green's function in which the higher order terms, up to any order $n$, and the error term are calculated and "Taylor expansions" of $f(z, \epsilon)$ and of its inverse $\phi(w, \epsilon)$ in powers of $\epsilon$ with estimate of the remainder term. (Received November 13, 1956.)

265t. Alexander Weinstein: Subharmonic functions and Tricomi equations.

Let $u^{k}\left(x_{1}, x_{2}, \cdots, x_{m}, r\right)=u^{k}(x, r)$ be the solution of the singular Cauchy problem for the Euler-Poisson-Darboux equation with $k \geqq 0$, corresponding to the initial values $u^{k}(x, 0)=f(x)$. The following results have been established by the use of the formulas for $u^{k}$ and by the fact that $u^{k}$ is also a solution of a generalized Tricomi equation (A. Weinstein, Communications on Pure and Applied Mathematics, Vol. VII, pp. 105-116, 1954). Let $M(x, r, f)$ be the mean value of $f$. For $k \geqq m-1$ the maximum and the minimum of $u^{k}$ are attained for $r=0$. Assuming the conditions $\partial^{i} M / \partial r^{i} \geqq 0, i=1,2, \cdots, n ; n \geqq(m-1-k) / 2$ (which are satisfied if the iterated Laplacians $\Delta^{i} f \geqq 0$ ) for $0 \leqq k<m-1$, then the minimum of $u^{k}$ is attained for $r=0$. Moreover if $\partial M / \partial r \geqq 0$ for $k \geqq m-1$ and if $\partial^{i} M / \partial r^{i} \geqq 0, i=1,2, \cdots, n+1$ for $0 \leqq k<m-1$ then $\partial u^{k} / \partial r \geqq 0$. Under the same conditions $u^{k}$ is a convex function of $r^{1-k}, k \neq 1$, and a convex function of $\log r$ for $k=1$. These results include the classical theorems on subharmonic functions. (Received November 5, 1956.)

\section{6t. G. L. Weiss: On certain spaces of analytic functions.}

Let $\Phi \neq 0$ be a non-negative, nondecreasing, convex function on the entire real line such that $\Phi(x) \rightarrow 0$ as $x \rightarrow-\infty$. The space $H_{\Phi}$ is then defined to be the class of all functions $F(z)$, analytic in the circle $|z|<1$, such that $\mu_{\Phi}(r ; F)=(1 / 2 \pi)$ - $\int_{0}^{2 \pi} \Phi\left(\log \left|F\left(r e^{i x}\right)\right|\right) d x$ is bounded for $0 \leqq r<1$. These function spaces generalize $H_{p}$ spaces in the same direction that Orlicz spaces generalize $L_{p}$ spaces. Using $\|F\|_{\Phi}$ $=\inf \left[|\lambda| ; \mu_{\Phi}(r ;(1 / \lambda) F) \leqq 1\right.$ for $\left.0 \leqq r<1\right]$ as a generalization of the $H_{p}$ norm, one can show that many of the well known properties of $H_{p}$ spaces, $p>0$, are satisfied by $H_{\Phi}$ spaces. (Received November $\left.5,1956.\right)$

\section{John Wermer: Function rings on the circle.}

Let $C$ be the normed ring of all continuous complex-valued functions on the unit circle $S_{0}$ and let $A$ be a closed subring of $C$ which separates points on $S_{0}$ and contains 
all constants. $A$ is said to be of type $R$ provided there exists a homeomorphism $\chi$ of $S_{0}$ on a simple closed curve $\gamma$ which lies on a Riemann surface $F$ such that $\gamma$ bounds a region $D$ on $F$ with $D \bigcup_{\gamma}$ compact and for each $g$ in $A$ the function $g\left(\chi^{-1}\right)$ defined on $\gamma$ is the boundary function of a function analytic in $D$ and continuous in $D \cup_{\gamma}$. A ring of type $R$ cannot equal all of $C$. Let now $\phi$ and $f$ be a pair of functions in $C$ which together separate points on $S_{0}$ and denote by $A(\phi, f)$ the smallest closed subring of $C$ containing $\phi, f$ and 1 . Assume that $\phi$ and $f$ are both analytic at each point of $S_{0}$, that $\phi^{\prime} \neq 0$ everywhere on $S_{0}$, and that $\phi$ does not map distinct arcs on $S_{0}$ onto the same arc. Theorem: Under these hypotheses, either $(A \phi, f)=C$ or $A(\phi, f)$ is of type $R$. (Received November 13, 1956.)

\section{Harold Widom: Extreme eigenvalues of Toeplitz matrices.}

Let $f(\theta) \sim \sum c_{n} e^{i n} \theta$ be real and continuous, and assume $f(\theta)$ attains its maximum $M$ at a unique point $(\bmod 2 \pi)$, say at $\theta=0$. Assume further that $f(\theta)$ is even, has a continuous fourth derivative in a neighborhood of $\theta=0$, and that $f^{\prime \prime}(0) \neq 0$. Let the eigenvalues of the Toeplitz matrix $\left(c_{i-1}\right)(i, j=0, \cdots, n)$ be $\lambda_{1, n} \geqq \lambda_{2, n} \geqq \cdots \geqq \lambda_{n+1, n}$. Then for fixed $k, \lambda_{k, n}=f\left(k \pi(n+2)^{-1}+k \pi\left(\alpha+\epsilon_{n}\right)(n+2)^{-1}\right)$, where $\epsilon_{n} \rightarrow 0$ as $n \rightarrow \infty$ and $\alpha=1+(2 \pi)^{-1} \int_{0}^{\pi} \csc ^{2} \theta / 2 \cdot \log \left[\left(2 f^{\prime \prime}(0)\right)^{-1}(f(\theta)-M) \cot ^{2} \theta / 2\right] d \theta$. (Received October 26, 1956.)

269t. J. C. Wilson: $A$ note concerning an nth order perturbed equation.

A sufficient condition is obtained whereby one is assured that there exists, for each value of $\mu$, a unique solution, through $n$ points, of the equation $x^{(n)}+\sum_{i=1}^{n} a_{i}(t) x^{(i-1)}$ $=\mu f\left(t, x, \cdots, x^{(n-1)}\right), \mu$ small. The sufficiency is shown to be equivalent to the uniqueness of a solution of the associated homogeneous equation through these same points. The method of obtaining the result employs standard procedures met with in the study of perturbed systems of differential equations. (Received October 25, 1956.)

270. Mishael Zedek: Strong infra- $(n, s)$-polynomials. Preliminary report.

Given a compact point set $E$ in the $z$-plane, integers $n$ and $s(0 \leqq s<n)$ and complex numbers $A_{1}, A, \cdots, A_{s}$, the polynomial $S_{n}^{s}(z) \equiv z^{n}+A_{1} z^{n-1}+\cdots+A_{8} z^{n-\text { 。 }}$ $+a_{s+1} z^{n-s-1}+\cdots+a_{n}$ is called a strong infra- $(n, s)$-polynomial on $E$, if it has no weak underpolynomial on $E$, that is if there is no other polynomial of the form $P_{n}^{\prime}(z) \equiv z^{n}+A_{1} z^{n-1}+\cdots+A_{s} z^{n-s}+c_{s+1} z^{n-a-1}+\cdots+c_{n}$, such that $\left|P_{n}^{\prime}(z)\right|$ $\leqq\left|S_{n}^{*}(z)\right|$ for $z$ on $E$. Generalized Tchebycheff polynomials $T_{n}^{8}(z)$ (compare: Walsh and Zedek, Proc. Nat. Acad. Sci. vol. 42 (1956) pp. 99-104) are strong infra-(n, s)polynomials. If $E$ is a real pointset, containing at least $n-s$ points and if $A_{1}, \cdots, A_{8}$, are real, then all the other coefficients of $S_{n}^{*}(z)$ are also real and at least $n-s$ of its zeros lie on the smallest segment containing $E$. This is a generalization of a theorem by Professor Walsh (same reference). If $E$ is symmetric with respect to the real axis and the prescribed coefficients of the generalized Tchebycheff polynomial $T_{n}^{*}(z)$ are real, then the rest of its coefficients are real. The nonreal zeros of $T_{n}^{1}(z)$ in this case are on the union of all ("Jensen") discs having as diameter the segment joining a nonreal point of $E$ with its conjugate (compare: Fekete and von Neumann, Jber. Deut. Math. Ver. vol. 31 (1922) pp. 125-138). (Received November 13, 1956.)

271t. David Zeitlin: Plane parabolic functions. Preliminary report.

Given $y^{2}=2 p x$, one defines the signed parabolic sector area, $A=\int_{0}^{-x} y d x$ $-2^{-1}(x-p / 2) y$, the parabolic radian, $u=8 A / p^{2}=2(y / p)+2(y / p)^{3} / 3$ and the six para- 
bolic functions: $\sin p(u)=y / p, \operatorname{cosp}(u)=2 x / p, \operatorname{tanp}(u)=\operatorname{sinp} u / \operatorname{cosp} u=p / y, \operatorname{cotp}(u)$ $=y / p$, secp $(u)=p / 2 x \operatorname{cscp}(u)=p / y$. Then $u=2 \operatorname{sinp} u+2 \operatorname{sinp}^{3} u / 3 \operatorname{sinp}(-u)$ $=-\operatorname{sinp}(u), \operatorname{cosp}(-u)=\operatorname{cosp}(u), \operatorname{sinp}^{2} u=\operatorname{cosp} u$, and for $-\infty<u<\infty,-\infty$ $<\operatorname{sinp} u, \tan \mathrm{p} u, \operatorname{cotp} u, \operatorname{cscp} u<\infty$ and $0 \leqq \operatorname{cosp} u, \operatorname{secp} u<\infty$. If $u=(4 \tan \theta) / 3$ $-90^{\circ} \leqq \theta \leqq 90^{\circ}$, then $\operatorname{sinp}((4 \tan \theta) / 3)=(\tan \theta+\sec \theta)^{1 / 3}+(\tan \theta-\sec \theta)^{1 / 3}$. One finds that $\operatorname{sinp} u \sim(3 u / 2)^{1 / 3}$ as $|u| \rightarrow \infty$ and that $\lim _{u \rightarrow 0} \operatorname{sinp} u / u=1 / 2$. The single valued inverse functions are arcsinp $u=\operatorname{arccotp} u=2 u+2 u^{3} / 3 \operatorname{arccscp} u=\operatorname{arctanp} u=2 / u$ $+2 / 3 u^{3}$, and the double valued inverses are arccosp $u= \pm\left(2 u^{1 / 2}+2 u u^{1 / 2} / 3\right)$ and arcsecp $u= \pm\left(2 / u^{1 / 2}+2 / 3 u u^{1 / 2}\right)$. If $\operatorname{sinp} u=\sum_{n-0}^{\infty} a_{n} u^{n}$, then $a_{2 n}=0$ for all $n, a_{1}=1 / 2$, and for odd $n, a_{n}+3^{-1} \sum_{m=0}^{n} \sum_{p=0}^{m} a_{p} a_{n-m} a_{n-p}=0$. The solution of the eq. $2\left(1+y^{2}\right) d y$ $=d u,(0,0)$, is $y=\operatorname{sinp} u$. One finds that $\left(\operatorname{sinp} u_{1}\right)\left(\operatorname{sinp} u_{2}\right)= \pm 1$, where 1 is for any family of straight lines through $(p / 2,0)$, and -1 for any family of straight lines through $(-p / 2,0)$. The derivatives and integrals of parabolic functions are found. A useful trigonometry is not apparent. (Received November 2,1956.)

\section{A. D. Ziebur: Separation and comparison theorems for differen- tial equations of higher order.}

Theorems analogous to the familiar results of Sturm on the oscillations of solutions of self-adjoint differential equations of the second order are developed for equations of higher order. They are proved with the aid of certain properties of the eigenvalues of an associated differential problem. There is a close connection with the work of Leighton [Bull. Amer. Math. Soc. vol. 55 (1949) pp. 325-328] on the second order case. (Received October 15, 1956.)

\section{Applied Mathematics}

\section{M. I. Aissen: Set functions of convex domains.}

In Isoperimetric inequalities in mathematical physics, Polya and Szego use a geometric property of plane convex domains called the "Inclusion Lemma" to derive bounds and approximations for various set functions of plane convex domains. In the discussion, the radii of the least circumscribing and the greatest inscribed circles play a useful role. In this paper the Inclusion lemma is proven for $n$-dimensional convex domains and is applied to their set functions. The geometric quantities which play the role of the inradius and circumradius are also discussed. (Received November 13, 1956.)

\section{R. J. Arms: Truncation errors involved in using special differ-} ence equations. Preliminary report.

Partial differential equations under consideration were of the elliptic type. A study was made of the error (i.e., the difference between the true solution and the difference solution) induced by the use of somewhat inaccurate difference equations along interior curves across which there was a change in "mesh size." Also the use of inaccurate difference equations along irregular boundaries was considered. By extending theorems of Gerschgorin (Z. Angew. Math. Mech. vol. 4 (1930) pp. 373-382) it was found that the above mentioned types of inaccuracies did not, in many usual situations, change the order of the error. The theorem proven, as that of Gerschgorin, contains assumptions on the differentiability of the true solution. (Received November 8,1956 .) 
275. E. H. Bareiss: Comparison of the theoretical accuracy of the $D T M B$ transport theory codes with the orthogonal polynomial approximations and $S_{n}$-approximations.

A survey is given of three general methods for the actual numerical solution of the Boltzmann Transport Equation. It is shown under which conditions the method of expanding in orthogonal polynomials can be made equivalent to the method of discrete ordinates. A generalization of the $S_{n}$-method is suggested in order to obtain the same theoretical accuracy as for the methods mentioned above.

The results of actual numerical computations are exhibited in graphical form. More details, also concerning the space integration, can be found in the report: Some theoretical aspects of the numerical solution of the one-dimensional Boltzmann transport equation, AML technical report Number 20, December 1956. (Received November 13, 1956.)

276t. A. T. Bharucha-Reid: Semi-groups as solutions of the Schrödinger equation. Preliminary report.

Recently semi-group theory has been used rather extensively in the study of Markov processes. While the probability structure of quantum theory differs from that of Markov processes, the quantum mechanical kernel function $K(t, x ; y)$ satisfies the Chapman-Kolmogorov equation $K(s+t, x ; y)=\int K(s, y ; d \xi) K(t, x ; \xi)$ which expresses the semi-group property; and the probability amplitude function $\Phi(t, x)$ satisfies $\Phi(s+t, x)=\int \Phi(s, \xi) K(t, x ; d \xi)$. Expansion of $\Phi(s+t, x)$ yields the Schrödinger equation $\Phi_{t}=\Omega \Phi$, where $\Omega \cdot=(i k / 2 m)\left(\partial^{2} \cdot / \partial x^{2}\right)+(i / k)(E-V(x)) \cdot$. In this paper we investigate the semi-groups which arise as solutions of the Schrödinger equation when various potential functions $V(x)$ which are of physical interest are introduced in the operator $\Omega$. (Received November 13, 1956.)

277. R. A. Clark (p) and W. E. Gibson: Asymptotic solution of a non-homogeneous differential equation. Preliminary report.

The problem is to determine an asymptotic solution of a differential equation of the form $u^{\prime \prime}(t)+\left[\lambda^{2} \phi(t)+\lambda \chi(t, \lambda)\right] u=\psi(t, \lambda)$ where $\lambda$ is a large complex parameter, $\phi$ has a single zero of first order, and $\chi(t, \lambda)=\sum_{n=0} \lambda^{-n / 3} \chi_{n}(t), \psi(t, \lambda)=\sum_{n-0} \lambda^{-n / 3} \psi_{n}(t)$ with $\chi_{n}$ and $\psi_{n}$ regular functions of a real variable $t$ over a closed interval including the zero of $\phi$. A formal solution is derived by two different methods. One method leads to an expansion of the form $u=\lambda^{-4 / 3} \sum_{n=0} \lambda^{-n / 3} \sum_{k=0}^{n} A_{n, k}(t) S^{(k)}(z)$ where $z=(2 \lambda \omega / 3)^{2 / 3}$ with $\omega=\int_{0}^{\phi} \phi^{1 / 2} d t, S$ is a solution of $S^{\prime \prime}(z)+z S=1$, and $S^{(k)}$ is the $k$ th derivative of $S$ with respect to $z$. The other results in a solution analogous to that obtained by R. E. Langer [Trans. Amer. Math. Soc. vol. 67 (1949) p. 461] for the corresponding homogeneous equation. When $\lambda$ is restricted to appropriate sectors of the complex $\lambda$-plane, $S$ may be chosen so that the formal solution is an asymptotic expansion in $\lambda$ of an exact solution. (Received November 13, 1956.)

278. H. J. Ettlinger (p) and J. L. Cornette. A reduced linear system from organic chemistry.

Let $C(t)$ be an $(n+1) \times(n+1)$ matrix of real integrable functions on $0 \leqq t \leqq T$, $T>0$, and $x(t)$ an $(n+1) \times 1$ matrix of real functions $x_{i}(t)$, absolutely continuous on $0 \leqq t \leqq T$. Consider $x(t)=x_{0}+\int_{0}^{t} C x$ where $x_{0}$ is a column of $n+1$ real numbers, $x_{i 0}$, 
such that $0 \leqq x_{i 0} \leqq 1$. Further, $c_{i i}(t) \leqq 0, c_{i j}(t) \geqq 0, i \neq j$, sum $c_{i i}(t) \leqq-k, k$ a positive number, on $t \geqq 0$ and sum $x_{i}(t) \equiv 1$ on $t \geqq 0$. This system is reduced by elimination of $x_{n+1}(t)$ to $x(t)=x_{0}+\int_{0}^{t}[A x+b]$ where $A$ and $b$ are readily identified The asymptotic and growth properties of $x_{i}(t)$ are discussed. (Received November 9, 1956.)

279. C. L. Dolph (p) and D. C. Lewis: On the application of infinite systems of ordinary differential equations to perturbations of plane Poiseuille flow.

In this paper the authors present an outline of their adaptation and application to the problem of the title of the method used by E. Hopf (Mat. Nach., 4, vol. 1-6, 1951, pp. 213-231) to establish the existence of a weak solution to the Navier-Stokes equations and a resume of the numerical results obtained from it to date on the Ramo-Wooldridge 1103 Computer. A solution of the linearized stream equation is sought in the form $\psi(x, y, t)=\exp (i \alpha x) \sum_{1}^{\infty} a_{n}(t) \phi_{n}(y)$ where the functions $\left\{\phi_{n}(y)\right\}$ are the known eigensolutions of a prescribed free-free beam problem and where the functions $\left\{a_{n}(t)\right\}$ satisfy the infinite system of ordinary linear differential equations with constant coefficients which results when the above expansion is inserted into the Navier-Stokes equations and sampled by the complete other normal family $\left\{\phi_{n}(y)\right\}$. The eigenvalues of this infinite system are shown to be proportional to those of the Orr-Semmerfield equation. Numerical computations indicate instability is present in a truncated six by six system and the truncated eight by eight system yields a curve of neutral stability with minimum critical Reynolds number of the right order of magnitude. Preliminary calculations with a twenty by twenty system indicate good agreement with the results of Lin. (Received November 13,1956.)

\section{Avron Douglis: An ordering principle and weak solutions of a quasi-linear partial differential equation.}

A "weak" solution in the half-plane $t \geqq 0$ of a quasi-linear partial differential equation in two independent variables of the form $u_{t}+F(u)_{x}=0$ is a solution in an integral sense satisfying the "entropy" condition $u(x-0, t) \geqq u(x+0, t)$ for $t>0$. Given any step function $\phi(x)$, a weak solution is easily constructed such that $u(x, 0)=\phi(x)$. Such a weak solution we call a "reference" solution. Under the hypothesis that $F^{\prime}(u)$ is a bounded, nondecreasing function, reference solutions are partially ordered like their initial data, or, more precisely, if $u_{1}$ and $u_{2}$ are two reference solutions such that the inequality $u_{1} \geqq u_{2}$ holds for $t=0$, then the inequality holds for $t>0$ as well. This ordering property makes it possible by simple monotonic processes to construct weak solutions for integrable initial data. The ordering property, moreover, prevails in a much larger class of weak solutions than is constituted by the reference solutions and their limits, and, therefore, uniqueness theorems can be proved for weak solutions of certain types. In this way, in particular, it is seen that smooth solution, when it exists, can be obtained as the monotonic limit of reference solutions. The existence theorems obtained by this procedure are comparable with those of P. D. Lax and the uniqueness theorem with that of $\mathrm{O}$. Oleinik. The ordering principle is believed to be new. (Received November 13, 1956.)

281. G. E. Forsythe (p) and Peter Henrici: Proof of convergence of the cyclic Jacobi iterative process for computing eigenvalues of a real symmetric matrix.

Let $A^{(0)}=\left(a_{i j}^{(0)}\right)$ be a real symmetric matrix of order $n$ whose eigenvalues $\lambda_{\alpha}$ are desired. For $k=0,1, \cdots$, let $A^{(k-1)}=U_{i j}^{T} A^{(k)} U_{i j}$, where $U_{i j}=U_{i j}^{(k)}$ is a rotation of 
the $(i, j)$ coordinate 2-plane through an angle $\theta_{i j}=\theta_{i j}^{(k)}$ chosen so that $a_{i j}^{(k+1)}=0$. C. G. J. Jacobi [J. Reine Angew. Math. vol. 30 (1846) pp. 51-95] proved that one can compute $\lambda_{\alpha}$ iteratively by the relation $\left(\lambda_{\alpha} \delta_{\alpha \beta}\right)=\lim _{k \rightarrow \infty} A^{(k)}$, where at each stage the couple $(i, j)$ is chosen so that $\left|a_{i j}\right|=\max _{p \neq q}\left|a_{p q}\right|$. But in current automatic digital computer practice [R. T. Gregory, Math. Tables Aids Comput. vol. 7 (1953) pp. 215-220] one often selects the $(i, j)$ in the cyclic order $(1,2),(1,3), \cdots,(n-1, n)$, and repeat. Convergence of such a "cyclic Jacobi process" is now proved, apparently for the first time. The key to the proof is the choice of $\theta_{i j}$ so that $-\pi / 4 \leqq \theta_{i j} \leqq \pi / 4$. If one chooses $0 \leqq \theta_{i j}<\pi / 2$, an example $(n=3)$ is given for which the cyclic Jacobi process failsboth with exact and rounded arithmetic operations. The result for hermitian $A^{(0)}$ is proved analogously. Proofs are elementary. (Sponsored by Office of Naval Research and Office of Ordnance Research.) (Received November 5, 1956.)

\section{W. T. Guy, Jr.: On the Mathieu transform. II.}

An integral transform involving Mathieu functions in the kernel is defined. Several properties are developed and used for working a certain class of differential equations. (Received November 13, 1956.)

\section{M. A. Hyman: Concerning the numerical solution of boundary- value problems.}

Attention is focused on the solution of elliptic partial differential problems, such as occur in elasticity, fluid mechanics, and nuclear reactor design. Usually finitedifference equations are introduced, which are then solved by some iterative technique. This paper proposes to use Fox's method (Proc. R. S. London vol. A190 (1947) p. 31), for setting-up the difference equations. Advantages are (1) a relatively coarse mesh can be used (thus speeding convergence of the iterative solution) associated with a small truncation error; (2) irregular boundaries, the use of finer meshes in certain regions, etc. can be handled without using complicated difference approximations or introducing sizeable truncation errors. It is also shown how, by a certain weighted averaging process, a starting approximate solution is obtained which is largely free of the "fundamental" component of the error, which decays most slowly during iteration and determines the rate of convergence. It is pointed out that the "over-relaxation factor" used during iteration, and usually difficult to estimate, is a domain functional for the partial difference operators employed. One can therefore expect to get good estimates using analogs of recently developed methods for partial differential operators. The paper concludes with certain remarks about extrapolation techniques for speeding convergence. (Received September 10,1956.)

\section{4t. M. A. Hyman: Line over-relaxation for solving partial differ- ence equations. Preliminary report.}

Independently Young and Frankel have discovered the effectiveness for solving elliptic partial difference equations of what is here called (modifying Young's terminology) "successive point over-relaxation" (SPOR). Recently Peaceman, Rachford and Keller, Rose have considered two different forms of "successive line over-relaxation" (SLOR). Regarding an iteration (during which each field-point is "swept" once) as a "time-step," relaxation processes such as those above are seen as the orderly "step-wise" solution of certain partial difference equations, first-order (usually, though higherorder equations are possible) in the added variable "time." Stability analysis then yields certain restrictions on the over-relaxation factors and indicates how various components of the error change under relaxation. In particular, the Young-Frankel 
and Keller-Rose techniques are so studied. The Peaceman-Rachford technique is always stable provided that directions are alternated in traversing the domain; this restriction is usually inconvenient when using automatic computing machines. Certain modifications of the Peaceman-Rachford technique are suggested which tend to avoid this difficulty without causing significant "instability." Finally, it is shown that the Peaceman-Rachford technique, so modified, can be used to solve "transport" problems-in particular those of neutron-transport, usually attacked now with multigroup methods and point-relaxation techniques. (Received September 20, 1956.)

\section{5t. M. A. Hyman: Finite-difference solution of the heat conduction equation (Variable time increments).}

The simplest implicit finite-difference approximant to the partial differential equation $u_{t}=u_{x x}$ is $\left({ }^{*}\right) w(x, t)-w(x, t-\Delta t)=\left(\Delta t / \Delta x^{2}\right)[w(x-\Delta x, t)-2 w(x, t)+w(x+\Delta x, t)]$, proposed by the author [J. Math. Phys. vol. 23 (1951) p. 223]. $\left({ }^{*}\right)$ is stable for all $r \equiv \Delta t / \Delta x^{2}$. Douglas, Gallie [Proc. Amer. Math. Soc. vol. 6 (1955) p. 787] have considered numerical solution of $u_{t}=u_{x x}$ using $\left(^{*}\right)$ with certain simple initial and boundary conditions; $\Delta x$ is constant, but $\Delta t$ may vary with $t$. For $r=\alpha+\beta t$ and $r=e^{\gamma t}(\alpha>0, \beta \geqq 0$, $0 \leqq \gamma \leqq \pi^{2} / 2$ ), they have shown that the maximum truncation error $E \equiv u-w$ in $R$ (the bounded rectangular region within which the solution is sought) is $O\left(\Delta x^{2}\right)$; this implies convergence of $w$ to $u$ in $R$ as $\Delta x \rightarrow 0$. In the present paper, the above results are extended as follows: $E=O\left(\Delta x^{2}\right)$ provided that the mesh-ratio $r$ is bounded in $R$; the variation of $r$ with $t$ in $R$ can be arbitrary. $E \rightarrow 0$ (convergence) as $\Delta x \rightarrow 0$ if $\max _{R} \Delta t \rightarrow 0$; this is the best possible result. It appears that the methods used can be applied to give similar strong results for other "stepping-ahead" type problems, including other differential equations (not necessarily parabolic) and more complicated initial and boundary conditions. (Received September 20,1956.)

\section{A. B. Lehman: Lattice order of two-terminal resistor networks.}

Let $P\left(a_{1}, \cdots, a_{n}\right), Q\left(a_{1}, \cdots, a_{n}\right), R_{1}\left(a_{1}, \cdots, a_{n}\right), \cdots, R_{m}\left(a_{1}, \cdots, a_{n}\right)$ be twoterminal network configurations whose branches are $a_{1}, \cdots, a_{n}$. Let $\bigcup$ denote a series configuration and $\cap$ denote a parallel configuration. Let swfn $(P)$ denote the two-terminal switching function of $P$, and $Z(P)$ denote its two-terminal resistance. Theorem 1 : $\operatorname{swfn}\left(P\left(a_{1}, \cdots, a_{n}\right)\right) \supset \operatorname{swfn}\left(Q\left(a_{1}, \cdots, a_{n}\right)\right)$ if and only if $Z\left(P\left(a_{1}, \cdots, a_{n}\right)\right)$ $\geqq Z\left(Q\left(a_{1}, \cdots, a_{n}\right)\right)$ for all positive values of $Z\left(a_{1}\right), \cdots, Z\left(a_{n}\right)$. Corollary: $Z\left(P\left(a_{1} \cup b_{1}, \cdots, a_{n} \cup b_{n}\right)\right) \geqq Z\left(P\left(a_{1}, \cdots a_{n}\right) \cup P\left(b_{1}, \cdots, b_{n}\right)\right)$. Theorem 2: swfn $\left(P\left(a_{1}, \cdots, a_{n}\right)\right) \supset \operatorname{swfn}\left(\bigcap_{k} R_{k}\left(a_{1}, \cdots, a_{n}\right)\right)$ if and only if $Z\left(P\left(a_{1}, \cdots, a_{n}\right)\right)$ $\geqq Z\left(\bigcap_{k} R_{k}\left(a_{1}, \cdots, a_{n}\right)\right)$ for all positive values of $Z\left(a_{1}\right), \cdots, Z\left(a_{n}\right)$. Theorem 3: $\operatorname{swfn}\left(\bigcup_{k} R_{k}\left(a_{1}, \cdots, a_{n}\right)\right) \supset \operatorname{swfn}\left(Q\left(a_{1}, \cdots a_{n}\right)\right)$ if and only if $Z\left(\bigcup_{k} R_{k}\left(a_{1}, \cdots, a_{n}\right)\right)$ $\geqq Z\left(Q\left(a_{1}, \cdots, a_{n}\right)\right)$ for all positive values of $Z\left(a_{1}\right), \cdots, Z\left(a_{n}\right)$. Given real numbers $p \geqq r \geqq s \geqq q \geqq 0$ such that $p \leqq r+s+t$ and $1 / q \leqq 1 / r+1 / s+1 / t$ for some $t \geqq 0$, then a bridge type two-terminal two-switch (four-state) resistor network will realize the four driving point resistances $p, r, s, q$. Furthermore, the four driving point resistances $p \geqq r \geqq s \geqq q$ of any two-terminal two-switch resistor network must be such that $p \leqq r+s+2 t$ and $1 / q \leqq 1 / r+1 / s+2 / t$ for some $t \geqq 0$. The proof uses theorems (2) and (3). (Received November 13, 1956.)

\section{Emanuel Parzen: $A$ simple proof and some extensions of the sampling theorem.}

The sampling theorem states essentially that if the frequency spectrum, or Fourier transform, $g(\omega)$ of a time function $f(t)$ vanishes for $\omega$ outside some interval $I$, then 
$f(t)$ is completely determined by its values at certain discrete sampling points, whose density is proportional to the length of the interval $I$. This note gives a method of proof of the sampling theorem, both for the case where the interval $I$ is centered at the origin and where it is not, which is somewhat simpler than the previously given proofs, and at the same time is more rigorous, and yields several useful generalizations to functions of several variables and random functions. (Received November 14, 1956.)

\section{W. H. Peirce: Numerical integration over the spherical shell.}

In this paper is given a formula for numerical integration over the arbitrary spherical shell. The type of formula developed is a finite weighted sum of integrand values, and the formula is exact whenever the integrand function is a polynomial of arbitrarily high degree. The points at which the integrand function is evaluated are inside the region of integration. Several specific formulas are given, and a brief error analysis is included. (Received November 5, 1956.)

\section{W. P. Reid: Heat flow in a cylinder.}

A formal, analytical solution is obtained to the problem of finding the temperature in a cylinder which is gaining or losing heat by radiation exchange with a concentric, thin-walled metal tube. The tube in turn is radiating to, or receiving radiation from, its surroundings. Because of the boundary condition, the eigenfunctions for this problem are not orthogonal. The modified orthogonality relation which they satisfy is given. The application of Duhamel's theorem is lengthy in this problem, and so a procedure is used which avoids the need for it. (Received November 14, 1956.)

\section{George Seifert: Pendulum-type systems with periodic forcing.}

Consider the system $\dot{\theta}=y, \dot{y}=g(\theta)+p(t)-\alpha f(\theta)$, where $f, g$, and $p$ are of class $C_{1}$, $f(\theta)>0, f(\theta+2 \pi)=f(\theta), g(\theta+2 \pi)=g(\theta), p(t+T)=p(t)$, for all $\theta$ and $t$, and if $A=\max |p(t)|$, each function $g(\theta) \pm A$ has at least one simple zero. By means of a procedure similar to one used by Lillo and Seifert, (cf. ZAMP vol. 7 (1956) pp. 239243), bounded regions $R_{n}$ in the $(\theta, y)$ plane are constructed such that for $\alpha$ sufficiently large, each such region contains the trajectory of a solution of period $T$, and that each solution is ultimately bounded in either this region, which is of width less than $2 \pi$, or another explicit region of the same type. It is also found that for $\alpha$ sufficiently large, a sub-region of each $R_{n}$ containing a unique periodic trajectory which is stable with respect to all trajectories in this subregion may be constructed. (Received November 13, 1956.)

\section{1t. Deonisie Trifan: On a complementary energy principle.}

A minimal principle in terms of the differential of stress deviation for the so-called "stress theory of plastic flow" is established. The stress-strain relation for this theory is applicable to homogeneous, isotropic, incompressible, strain-hardening materials exhibiting a gradual transition from the elastic to the plastic state. It is proved that the actual differential of stress and corresponding strain deviations occurring in a body under external forces make a volume integral involving admissible differential stress deviations an absolute minimum. (Received November 13,1956.)

292. Herschel Weil: On the convergence of the Mie series for scattering spheres.

G. Mie's classical solution for the electromagnetic field scattered by a sphere irradiated by a plane wave consists of expansions in spherical vector wave functions of 
integral order, $n$. These series do not begin to converge until somewhat more than $a$ terms, where $a$ is the sphere radius in units of wavelength $/ 2 \pi$, and considerable numerical error can and has been made by not including sufficient terms beyond $n=a$. With the help of bounds on the combinations of cylinder functions involved, a function $N(a, p)$ is obtained with the property that if $N$ terms in the series are computed for a given $a$, the percentage error in the result will be less than $p . N(a, p)$ is independent of the coordinates of the field point (receiver) and is a good estimate of the smallest permissable number of terms for a given error $p$. (Received November 14, 1956.)

\section{GEOMETRY}

\section{Smbat Abian (p) and I. A. Barnett: Semi-covariants in projec-} tive differential geometry.

Consider the system of differential equations (1) $y_{i}^{(m)}+\sum_{a=1}^{m} \sum_{j=1}^{n} A_{q i j}(x) y_{j}^{(m-e}$ $=0$, and the continuous group of transformations (2) $y_{i}=\sum_{j=1}^{n} K_{i j}(x) \bar{y}_{j}$ and (3) $\tilde{x}=\xi(x)$. If a function $f\left(y_{i}^{(h)}, \cdots, A_{1 i j}^{(q+r-1)}, \cdots, A_{q i j}^{(r)}, \cdots\right),(h=0,1, \cdots, H \leqq m-1$, $q=2, \cdots, Q \leqq m, r=0, \cdots, R)$ remains unchanged by both (2) and (3), it is said to be a covariant; if unchanged by (2) only, a semi-covariant of height $H$, class $Q$ and order $R$ of (1). This paper deals with the representation theory of semi-covariants. It is proved that the matrices $F_{q, r}$ of dimension $n$ with elements $F_{q, r ; i j}$ and their covariant derivatives obtained from the semicanonical form $z_{i}^{(m)}+\sum_{q=2}^{r} \sum_{t, j, r-1}^{q, r} U_{i t} F_{q ; t} V_{j r} z_{r}^{(m-q)}$ $=0$ of (1) undergo a similarity transformation and the vectors $Y_{h}$ with their elements $y_{i}$ and their covariant derivatives undergo the transformation (2). Any semicovariant $f$ is a solution of the complete system of $n^{2}$ equations $S_{p k}(H, Q, R)$ $=\sum_{q-2}^{Q} \sum_{r=0}^{R} \sum_{i, j=1}^{n}\left(\partial f / \partial f_{q, r ; i j}\right)\left(F_{q, r ; i p} \delta_{j}^{k}-F_{q, r ; k j} \delta_{p}^{i}\right)-\sum_{h=0}^{B}\left(\partial f / \partial Y_{h ; p}\right) Y_{h ; k}=0$. The number of independent semi-covariants of height $H$, class $Q$ and order $R$ of (1) is found to be equal to the expression $(Q R+Q-R-2) n^{2}+(H+1) n$. The explicit expressions for the semi-covariants are obtained as functions of elements of matrices of the form $\left(Y_{0}, \cdots, Y_{H}, F_{q, r} Y_{h}, \cdots\right)^{-1} F_{q, r}\left(Y_{0}, \cdots, Y_{H}, F_{q, r} Y_{h}, \cdots\right)$. (Received November $8,1956$.

\section{Iacopo Barsotti: Repartitions on abelian varieties.}

Let $A$ be an $n$-dimensional normal variety over the algebraically closed field $k$; a (repartition) class on $A$ is a mapping $\mathfrak{b}$ such that (1) for each $(n-1)$-dimensional irreducible subvariety $X$ of $A, \mathfrak{b} X=x(X)+Q(X / A), x(X) \in k(A)$, and (2) $\mathfrak{b} X$ $=Q(X / A)$ for all, but finitely many, $X$ 's. The class $\mathfrak{b}$ is exact if there is an $x \in k(A)$ such that $\mathfrak{b} X=x+Q(X / A)$ for each $X$; it is closed if for each $P \in A$ there is an exact class $\mathfrak{a}$ such that $(\mathfrak{b}+\mathfrak{a}) X=Q(X / A)$ for all $X \ni P$. If $A$ is an abelian variety, let $Q, B_{0}$ be the $k$-modules of, respectively, the closed, and the exact classes; typical results are: (1) $B / B_{0}$ is a $k$-module of order $n$; (2) there is an isomorphism $\alpha$ of the $k$-module $\mathfrak{D}$ of the invariant derivations on $A$, onto $B / B_{0}$, such that, if $p \neq 0, \alpha\left(D^{p}\right)$ $=\alpha\left(D^{p}\right)$ for $D \in D$; (3) there is a homomorphism $\beta$ of $B / B_{0}$ onto $D_{2} / D_{1}+D_{c}$ (where $D_{i}, D_{e}$ are respectively the $k$-modules of the differentials of the $i$ th kind, or exact), whose kernel is 0 if $p=0$, or $\left(B / B_{0}\right)^{p}$ if $p \neq 0$; (4) each $\omega \in D_{2}$ is closed. If $A$ is the jacobian of a curve $C$, relations are easily established between $B / B_{0}$ and the operation $\mathfrak{b} \rightarrow \mathfrak{b}^{p}$ for repartitions on $C$, or the dual operation $\omega \rightarrow \omega^{1 / p}$ for differentials on $C$. (Received November 13, 1956.) 


\section{Eugenio Calabi: Closed, locally euclidean, 4-dimensional mani-} folds.

Let $M^{q}$ be a closed, flat, $q$-dimensional manifold ( $q$-fold), $G$ the (Compact Lie) group of all isometries of $M^{q}, r$ its first Betti number $(=\operatorname{dim}(G)=\operatorname{dimension}$ of space of autoparallel, global vector fields), $\Delta$ a finite, Abelian subgroup of $G$ with finite centralizer (i.e. leaving no autoparallel vector field in $M^{q}$ invariant) and of rank $\leqq p$. Then $\Delta$ has a faithful representation $\phi: \Delta \rightarrow \Delta^{\prime}$ into the translation group of $T^{p}$ (=p-dimensional, flat torus). Let $\Delta^{\prime \prime}$ be the graph of $\phi$ in $\Delta^{\prime} \times \Delta$, considered as a group of isometries of $T^{p} \times M^{q}$. Then the quotient space $M^{p+q}=\left(T^{p} \times M^{q}\right) / \Delta^{\prime \prime}$ is a closed, flat $(p+q)$-fold with first Betti number $=p$. Theorem 1: Every closed, flat $n$-fold $M^{n}$ with first Betti number $p>0$ can be constructed by the method just outlined; the manifolds $T^{p}, M^{q}(q=n-p)$ and the groups $\Delta, \Delta^{\prime}$ are uniquely determined within afine equivalence by the topological invariants of $M^{n}$. From the known classification of closed, flat $n$-folds $(n \leqq 3)$ it is possible to list all closed, flat fourfolds. Theorem 2: There exist exactly 31 topologically inequivalent closed, flat, orientable fourfolds (all with first Betti number $\geqq 1$ ) and, not counting those with first Betti number $=0,40$ nonorientable ones. (Received November 14, 1956.)

\section{Michael Goldberg: Rotors tangent to $n$ fixed circles.}

Every closed curve (a rotor) which remains tangent to $n$ fixed straight lines in a plane during a complete rotation of the curve is a special case of a family derived analytically by Meissner [Vierteljahrschrift der naturforschenden Gesellschaft, Zürich, vol. 54 (1909) pp. 309-329]. This paper considers the replacement of the straight lines by circles. This is a generalization of a problem of Lusternik [Uspekhi Matematicheskikh Nauk, vol. 1, parts 3-4 (13-14)(1946) pp. 194-195]. He posed the problem of the determination of all the rotating closed curves which remain tangent to three fixed circles. A partial solution (possibly complete) of this generalized problem is derived from the known rotors in regular polygons. A direct kinematic derivation is shown for these general rotors. Several special extremal cases are shown. (Received October 30, 1956.)

\section{7t. Donald Greenspan: On vertices in euclidean 3-space.}

A space curve $C: \mathrm{x}=\left[x^{1}(s), x^{2}(s), x^{3}(s)\right]$, is called a curve of type $I$ if and only if: (a) $x^{i}(s)$ is analytic for each $i=1,2,3$, (b) parameter $s$ represents arc length, (c) neither $K=0$ nor $\tau=0$, at any point of $C$, and (d) the curve is not spherical. A point $P$ of such a curve $C$ is defined to be a vertex if and only if the osculating sphere at $P$ has at least five consecutive points in common with $C$ at $P$. A stationary point $Q$ of $C$ is a point where $d r / d s=0$, where $r$ is the radius of the osculating sphere. It is then shown that every vertex point is a stationary point. Consideration of the helix $K=\tau=1+s^{2}$, at $s=0$, reveals that a stationary point need not be a vertex. (Received November 1, 1956.)

\section{Frank Harary: On arbitrarily traversable graphs and directed} graphs.

A new proof of Bäbler's unique prime decomposition theorem for arbitrarily traversable graphs is given. This proof serves to characterize a prime arbitrarily traversable graph as a maximal connected subgraph of an arbitrarily traversable graph, with no cut points of itself. In addition, the theorems of Ore and Bäbler are extended to directed graphs. (Received November 14, 1956.) 
299. M. Herzberger (p) and E. W. Marchand: Elementary linear and differential geometry in four-space.

Since the physical laws can be considered as geometry in space-time, the authors feel that the transformation and variation of well known three-dimensional laws and methods to four-space merit interest. Problems of spherical trigonometry, elementary geometry, and differential geometry are discussed in four-space. Literature: $\mathrm{H}$. Grassman, Die lineale Ausdehnungslehre, O. Wigand, Leipzig, 1878. H. Forder, The calculus of extension, Cambridge University Press. E. W. Marchand, Spherical trigonometry in $n$-space, Dissertation, Rochester, 1952. M. Herzberger, Einige ïber lineare transformationen und Vektoranalysis in n-Dimensionen, Z. Angew. Math. Mech. vol. 11 (1931) pp. 431-433. (Received November 14, 1956.)

\section{C. C. Hsiung: A uniqueness theorem on two-dimensional Riemannian manifolds.}

Let $M^{2}$ and $M^{* 2}$ be two orientable two-dimensional Riemannian manifolds of class $C_{3}$ imbedded in a Euclidean space $E^{m}$ of dimension $m>2$ with positive Gaussian curvature in every normal direction and closed boundaries $C$ and $C^{*}$ respectively. Suppose that there is a homeomorphism of $M^{2}$ and $M^{* 2}$ such that at corresponding points $M^{2}$ and $M^{* 2}$ have the same normal spaces of dimension $m-2$ and equal sums of the principal radii of curvature in every common normal direction, and such that at corresponding points $C$ and $C^{*}$ have the same tangent vectors and equal linear elements. Then $M^{2}$ and $M^{* 2}$ are transformed into each other by a translation. When $m=3$, this result is due to the author (Math. Zeit. vol. 64 (1955) pp. 41-46) and to E. B. Christoffel for closed $M^{2}$ and $M^{* 2}$. (Received October 30, 1956.)

\section{Wilhelm Klingenberg: Projective geometry with homomorphism.}

A projective space with homomorphism (abbr.: w.h.) $P_{3}$ is a set of points, in which two kinds of subsets, called lines and planes, are distinguished. Furthermore there is given a mapping $P_{3} \rightarrow \bar{P}_{3}$, called homomorphism, of the points, lines and planes of $P_{3}$ onto the points, lines and planes of a projective space (in the usual sense) $\bar{P}_{3}$, and the usual incidence properties between points, lines and planes of $P_{3}$ hold, whenever they hold between the images of these points, lines and planes in $\bar{P}_{3}$. It is well known, that $P_{3}$ may be represented by the submodules $\neq 0$ of the left vector space $\bar{K}_{s}^{4}$ of rank 4 over a field $\bar{K}$. Conversely, the set of submodules $\neq 0$ of $\bar{K}_{s}^{4}$, with arbitrary field $\bar{K}$, represents a projective space. Therefore fields will be called the characteristic coordinate structure of a projective space. As a generalization of this it will be proved, that the characteristic coordinate structure of a projective space w.h. is a (general) local ring $A$ (that is a ring $A$ with unit, which is not necessarily commutative, and there exists a greatest ideal $I \neq A$, which contains any left and any right ideal $\neq A$ ). $A / I$ is a field, isomorphic with the coordinate field $\bar{K}$ of $\bar{P}_{3}$. The onto homomorphism $A \rightarrow \bar{K}$ induces an onto homomorphism $A_{s}^{4} \rightarrow \bar{K}_{s}^{4}$, where $A_{s}^{4}$ is the left $A$-module of rank 4. $P_{3}$ is represented by those submodules of $A_{s}^{4}$, which are not mapped into 0 under $A_{s}^{4} \rightarrow \bar{K}_{.}^{4}$. (Received October 9, 1956.)

\section{Lawrence Markus: Complete and convex affine connections.}

Several results are noted on the geometry of the geodesics for various complete and convex affine connections. (Received November 13,1956.) 
303. W. O. J. Moser: On the number of ordinary lines determined by $n$ points in the plane.

Let $n$ points, not all collinear, in the real projective plane be joined in all possible ways by straight lines. A connecting line which contains exactly 2 of the given points is called an ordinary line. Let $m=m(n)$ be the minimum number of ordinary lines determined by any set of $n$ noncollinear points. A famous conjecture of Sylvester (Educational Times, 59, 1893: 98) may be expressed in the form $m \geqq 1$. This was proved by Gallai in 1933 (see de Bruin and Erdoss, Nederl. Akad. Wetensch., Proc. 51: 1277-1279) and Steinberg (Problem 4065, Amer. Math. Monthly vol. 51 (1944) p. 169). Motzkin (Trans. Amer. Math. Soc. vol. 70 (1951) p. 451) showed that $(m+1)(m+2) \geqq 2 n$ and the author (Minutes Proc. Royal Soc. Canada, 1955, Appendix C, abstract 59:23) showed $2 m(m+5) \geqq 5 n$. In this paper it is shown that $m>(n+11) / 6$ when $n$ is even. (Received October 22, 1956.)

\section{T. K. Pan: Isometric mapping of two surfaces.}

Let $S$ and $\bar{S}$ be isometric surfaces associated with unit vector fields $v$ and $\bar{v}$, respectively. Let $P$ and $\bar{P}$ be their corresponding points. This paper is to investigate: (1) the existence of $S$ and $\bar{S}$ such that the curves and the lines of curvature of $v$ and $\bar{v}$ at $P$ and $\bar{P}$ are corresponding; (2) the existence of $\bar{S}$ which can be mapped isometrically onto a given $S$ such that the curves and the lines of curvature of $v$ and $\bar{v}$ at $P$ and $\bar{P}$ are preserved. It includes as special cases some problems studied by Bonnet and Cartan. Exterior differential calculus and method of moving trihedral are employed for this investigation. It is found that surfaces associated with unit vector fields are mapped isometrically with the curves and lines of curvature of the vector fields in correspondence if and only if they are mapped isometrically with the curves and asymptotic lines of the vector fields in correspondence. The general solution of (1) depends on one arbitrary function of two variables and that of (2) on two arbitrary functions of one variable. The nature of singular solution, the characteristic, and the problem of Cauchy are studied. (Received November 13, 1956.)

\section{E. J. Schweppe: A projective generalization of convexity.}

Consider a projective space $P$ which satisfies the Postulate of Fano and the property of the complete quadrangle. A set $Q$ of points in $P$ is called quasi convex if at least one of the points $C, D$ is in $Q$ whenever $[A, B ; C, D]$ is a harmonic quadruple and $A, B$ are in $Q$. If no points of $Q$ lie on some fixed hyperplane $H$, then $Q$ is a quasi convex subset of the affine space $P-H$. It is easily verified that classical convex subsets of affine spaces are also quasi convex subsets; furthermore, examples may be constructed of quasi convex subsets in projective spaces over fields which cannot be ordered. In particular, the points in the projective plane over the complex numbers such that $z_{1} \bar{z}_{1}+z_{2} \bar{z}_{2}-z_{3} \bar{z}_{3}<0$ form a quasi convex set. When the dimension of $P$ is greater than two, it is shown that the intrinsic properties of a quasi convex set $Q$, which spans $P$, completely determine the structure of $P$. This result generalizes the work of H. Kneser [Konvexe Räume, Archiv der Math. vol. 3 (1952) pp. 198-206] and M. H. Stone [Postulates for the barycentric calculus, Annali di Matematica vol. 29 (1949) pp. 25-30]. (Received November 13, 1956.)

306. Ernst Snapper: Cohomology groups and genera of higher dimensional fields.

Let $k \subset E$ be fields, where $E=k\left(e_{1}, \cdots, e_{n}\right)$, for $e_{1}, \cdots, e_{n} \in E$. The Riemann manifold $S$ of $E / k$ is the limit of the inverse system $\left.\left\{V_{i} ;\right\rangle\right\}$ of its projective models 
$V_{i}$ (O. Zariski, Bull. Amer. Math. Soc. vol. 50 (1944) pp. 683-691). We replaced each $V_{i}$ by its sheaf of local rings $\left(V_{i}, L_{i}\right)$ (J. P. Serre, Ann. of Math. vol. 61 (1955) pp. 197-278). The system $\left\{\left(V_{i}, L_{i}\right) ;>\right\}$ has as limit a sheaf of rings $(S, L)$ over $S$ as base space. For $x \in S$, the stalk $L_{x}$ is isomorphic with the valuation ring corresponding to $x$. We called $(S, L)$ the sheaf of valuation rings of $E / K$. We defined the $q$ th cohomology group $H^{q}(E / k)$ of $E / k$ as the $q$ th cohomology group of $(S, L)$. Let $h^{q}(E / k)$ denote the dimension of the $k$-vectorspace $H^{q}(E / k)$. We proved that $h^{q}(E / k)=0$ when $q$ exceeds the degree of transcendency $r$ of $E / k$; that $h^{0}(E / k)$ is the field degree of $k^{*} k$, where $k^{*}$ is the algebraic closure of $k$ in $E$; and that $h^{r}(E / k)$ is finite. Geometric genus and irregularity of $E / k$ are defined as respectively $h^{r}(E / k)$ and $h^{1}(E / k)$. If all $h^{q}(E / k)<\infty$, the arithmetic genus of $E / k$ is defined as $\sum(-1)^{q} h^{q}(E / k)$, where $q=0,1, \cdots, r$. These purely field theoretic genera and allied notions are investigated in this paper. (Received November 13, 1956.)

\section{Andrew Sobczyk: Spatial families of lines.}

A family $F$ of lines in three-dimensional Euclidean space $E_{3}$ is representative if it contains exactly one line in each (unsensed) direction of $E_{3}$. A representative family, which has the further property that every pair of its lines intersect, is the pencil of all lines through a point. A representative family $F$ determines a mapping $V \rightarrow P$ on the unit sphere $S_{2} \subset E_{3}$ to $E_{3}$; for $V$ in $S_{2}$ the image $P$ is the point in which the line $L$ of $F$ parallel to vector $V$ pierces the tangent plane of $S_{2}$ at $V$. The family is continuous in case this mapping is continuous. The following theorems are proved concerning any continuous representative family $F$ : If $L$ is any line of $F$ and $\Omega$ is any plane oblique to $L$, then there exists a line $N \in F$ which intersects $L$ and is parallel to $\Omega$. For any pair of lines $L, M$ of $F$, either $L$ and $M$ intersect, or there is a third line $N$ of $F$ which intersects both $L$ and $M$. (Received November 13,1956.)

\section{S. R. Struik: Two Euclidean theorems, a by-product of their affine counterpart.}

An affine reflection is the same as the commonly known one, but the angle, under which the connection of the point $P$ and its image point $P^{\prime}$ intersects the mirror, is of course an arbitrary angle (and not usually a right angle). $P P^{\prime}$ can lie in a line or a plane (or a three-space in 4-space) intersecting the mirror $s$. In affine two-space two (in three-space three) reflections suffice to transform two equal triangles (tetrahedrons) into each other. The Euclidean counterpart of the theorem is simpler: One (resp. two) reflections suffice in two- (resp. three-) space, because the mutual position of the compared triangles (tetrahedrons) can be conveniently selected. The same construction yields the ratio of their areas (volumes), purely synthetically, in the event of inequality, a parallel vector pair. Hilbert's arithmetization of the problem of area in the "Grundlagen" is scrutinized as is Süss' ad hoc solution of the difficulties. The writer believes axiomatics and definition had to be modified along affine lines. Moreover, the dualistic nature of the affine reflection leads to additional, worthwhile theorems in two three, and higher dimensions. (Received November 14, 1956.)

309. J. S. Taylor: The use of projective coordinates in real representations of spaces of $n$ complex dimensions.

Among the real representations for a space of $n$ complex dimensions is that of using the real points of a flat space of $2 n$ dimensions. A weakness of such representa- 
tions has been the lack of one-to-one correspondence with respect to the infinite elements. With the use of projective coordinates of the complex space the correspondence becomes one-to-one throughout. Further light is thus thrown on the nature of the Argand plane representation of the complex space of one dimension, since the point at infinity is exhibited as an arbitrary point of the plane; in particular, it is observed that the coordinate lines of the Argand plane are to be considered as two families of mutually orthogonal circles, each family having a common tangent at the infinite point. Of more importance, however, is the application of projective coordinates to the 4-space representation of the plane of two complex variables, where the line at infinity of the complex plane is represented by an arbitrary "regular" plane of the 4-space. This makes the representation completely one-to-one and resolves many difficulties previously existing. The generalization for spaces of $n$ complex dimensions is given in conclusion. (Received November 13, 1956.)

\section{LOGIC AND FoundaTIONS}

\section{M. D. Davis: An intrinsic definition of recursive functional.}

A definition of recursive functional of arbitrary type is given, in terms of partial recursiveness. For functionals of type ((1)1) this definition was known to G. Kreisel as a formalization of a definition of Brouwer. $x_{1}, g_{1}$, etc. range over the (natural) numbers; $\left.f_{\left(\alpha_{1} \alpha_{2}\right.} \ldots \alpha_{n}\right)$ ranges over functionals from $n$-tuples $\left(g_{\alpha_{1}}, \ldots, g_{\alpha_{n}}\right)$ to numbers. Numbers (including the "undefined" number) are of finite domain; $f$ is of finite domain if its domain consists of a finite set of $n$-tuples of functionals of finite domain. $f \subset h$ if the domain of $f$ is included in that of $h$ and they agree where defined. As $w$ takes on integral values, $w^{\alpha}$ ranges over all functionals of finite domain which are of type $\alpha$. If $f_{\alpha}$ is of finite domain, $\left\langle f_{\alpha}\right\rangle$ is the number such that $\left\langle f_{\alpha}\right\rangle_{\alpha}=f_{\alpha} \cdot x_{1}^{(\nu)}=x_{1}$ for $x \leqq y$ and is undefined otherwise; $f^{(y)}=f$ for argument $g_{\alpha}=g_{\alpha}^{(y)}$ if $f \leqq y$, otherwise $f^{(y)}$ is undefined. $\left.f_{\left(\alpha_{1} \alpha_{2}\right.} \cdots \alpha_{n}\right)$ is recursive if there is a partial recursive function $q\left(x_{1}, \cdots, x_{n}\right)$ such that if $q\left(w_{1}, \cdots, w_{n}\right)$ is defined, then $w_{i}^{\alpha_{i}} \subset v_{i}^{\alpha_{i}}$ implies $q\left(v_{1}, \cdots, v_{n}\right)=q\left(w_{1}, \cdots, w_{n}\right)$ and $w_{i}^{\alpha_{i}} C g_{\alpha^{i}}$ implies $f\left(g_{\alpha_{1}}, \cdots, g_{\alpha_{n}}\right)=q\left(w_{1}, \cdots, w_{n}\right)$. We have $f_{\left(\alpha_{1} \alpha_{2} \cdots \alpha_{n}\right)}\left(g_{\alpha_{1}}, \cdots, g_{\alpha_{n}}\right)$ $=U\left(\mu y \mathfrak{I}_{n}\left(e,\left\langle g_{\alpha_{1}}^{(y)}\right\rangle, \cdots,\left\langle g_{\alpha_{n}}^{(y)}\right\rangle, y\right)\right)$, for primitive recursive $\mathfrak{T}_{n}$, an extension of Kleene's extended normal theorem. (Received December 27, 1956.)

\section{Joachim Lambek: Calculus of syntactic types.}

The methods of K. Ajdukiewicz [Studia philosophica vol. 1 (1935) pp. 1-27] and Y. Bar-Hillel [Language vol. 29 (1953) pp. 47-58] are further developed to obtain the types of English pronouns and articles. Pronouns are regarded as functors which transform predicates into sentences, articles as functors which transform nouns into pronouns. Subject and object pronouns have different types, because juxtaposition is noncommutative. A calculus of types was suggested by algebraic techniques due to G. D. Findlay and the present author. Basic types are $n$ (noun) and $s$ (sentence); types $x$ and $y$ give rise to compound types $x \cdot y, x / y, x \backslash y$. There is a transitive and reflexive relation $\rightarrow$ between types, satisfying two further postulates: (1) $(x \cdot y) \cdot z$ $\rightarrow x \cdot(y \cdot z)$, (2) $x \cdot y \rightarrow z$ if and only if $x \rightarrow z / y$, and their symmetric duals. Consider for example the sentences "John likes fresh milk" and "he likes it." Their types are $n \cdot((n \backslash s) / n) \cdot(n / n) \cdot n$ and $(s /(n \backslash s)) \cdot((n \backslash s) / n) \cdot((s / n) \backslash s)$ respectively. Both types reduce to $s$ by the arrow. The relationship between noun and subject pronoun is expressed by the formula $n \rightarrow s /(n \backslash s)$. (Received November 13, 1956.) 
312. Hartley Rogers, Jr.: Provable recursive functions. Preliminary report.

$P$ is a standard axiomatization of elementary number theory, assumed sound. $A(i, x, y)$ is a well-known formula-schema such that $A(i, x, y) \Leftrightarrow \phi_{i}(x)=y$. ( $\phi_{i}$ is partial recursive function of index $i$.) $f$ is a provable function ( $p$-function) if $(\boldsymbol{B} j)\left[f=\phi_{j}\right.$ and “ $(\forall x)(\exists y) A(j, x, y)$ " is provable in $P]$. p-one-one functions and $p$-permutations are similarly defined. (i) The $p$-permutations form a noninvariant subgroup of the recursive permutations. p-isomorphism types, p-one-one reducibility $\left(\leqq_{1}^{p}\right)$ and p-many-one reducibility $\left(\leqq_{m}^{p}\right)$ are defined. (ii) $p$-isomorphism types coincide with $\leqq_{1}^{p}$ degrees of unsolvability. (iii) Degrees under $\leqq_{m}^{p}$ form a semi-lattice; recursive sets fall into infinitely many degrees (a semi-lattice) with a minimum nontrivial element and no maximum element; creative sets form a semi-lattice of more than one degree (infinitely many $p$-isomorphism types) possessing a maximum element (also the maximum $p$-isomorphism type, and consisting of sets whose productive functions are $p$-functions). (iv) Completions of two sets of same Turing degree are $p$-isomorphic. (v) Theorems of any two sound systems of number theory of minimal strength are $p$-isomorphic (analogue to theorem of Myhill). (A system of number theory is sound if no false formula is provable.) (Received November 13, 1956.)

\section{Statistics and Probability}

\section{H. D. Block: On Stochastic approximation.}

Dvoretzky (Proceedings of the Third Berkeley Symposium on Mathematical Statistics and Probability) proved convergence of stochastic approximation methods in a setting general enough to include all previous results on convergence. The proof was simplified by Wolfowitz (Annals Math. Statist., December, 1956). Extending these ideas, the present paper proves convergence with probability one under weaker conditions and where the process takes place in any normed vector space. (Received November 13, 1956.)

314t. H. D. Block: Estimates of error for two modifications of the Robbins-Monro stochastic approximation process.

Dvoretzky [Proceedings of the Third Berkeley Symposium on Mathematical Statistics and Probability] found optimal bounds on the mean square error after $n$ steps, $V_{n}$, and the optimal choice of coefficients $a_{k}$ for the Robbins-Monro process when $\left(^{*}\right)$ the regression line is bounded between two straight lines with positive finite slopes. We modify the process by taking the average of $n_{k}$ observations at the $k$ th step and, assuming $\left({ }^{*}\right)$, find optimal values for $V_{n}, a_{k}, n_{k}$; similarly for the modification arising from the requirement $a_{k}=a$ we find optimal values of $V_{n}, a, n_{k}$. For a given total number of observations the optimal estimate of $V_{n}$ is the same for all three processes. A similar procedure is valid for certain types of mappings of a normed vector space. (Received November 13, 1956.)

315t. A. B. Clarke: On statistics independent of sufficient statistics.

Let $\{f(x ; \theta): \theta \in \Theta\}$ be a family of probability density functions of exponential type, $\Theta$ being a connected, open subset of $R^{k}$. Let $s=s\left(x_{1}, \cdots, x_{n}\right)$ be a nontrival $k$-dimensional sufficient statistic for $\theta$. THEOREM: If $\left(x_{1}, \cdots, x_{n}\right)$ is a sample from $a$ population having frequency function $f(x ; \theta), \theta \in \Theta$, and if $t=t\left(x_{1}, \cdots, x_{n}\right)$ is any statistic on this sample, then $t$ is independent of $s$ if and only if the distribution of $t$ is in- 
dependent of $\theta$. This generalizes a result by R. G. Laha (Proc. Amer. Math. Soc. vol. 1 (1956) pp. 172-174), for normal and gamma populations. (Received November 13, 1956.)

\section{Gopinath Kallianpur: Optimum filtering with finite data.}

The paper considers the following problem treated in a heuristic manner by Laning and Battin (Random process in automatic control): Let $Z(t)(0 \leqq t \leqq T)$ be the corrupted message which is the sum of the message $V g(t)$ and the Gaussian noise $y(t)$ with continuous covariance function $R(t, s)$. It is assumed that $V$ is a random variable independent of $y(t)$ and that $g(t)$ is a known, continuous function of $t$. Under the hypothesis that the integral equation of the first kind $\left(^{*}\right) \int_{0}^{T} R(t, s) p(s) d s=g(t)$ has a continuous solution $p(t)$ it is proved that the optimum filter which minimizes the expected squared error is of the form $F\left(\int_{0}^{T} Z(t) p(t) d t\right)$, where the function $F$ is easily calculated knowing the distribution of $V$. When the message function is of the form $\sum_{i=1}^{m} V_{i} g_{i}(t)$, the $V_{i}$ 's being independent r.V.'s and the $g_{i}$ 's continuous functions of $t$, it is shown that the optimum filter is a function of the statistics $\int_{0}^{T} Z(t) p_{i}(t) d t$ $(i=1, \cdots, m)$ where each $p_{i}(t)$ is a solution of an integral equation of the form $\left(^{*}\right)$. (Received November 15, 1956.)

\section{7t. D. G. Kendall: A note on Doeblin's central limit theorem.}

The proof of Doeblin's central limit theorem for Markov chains is modified so as to yield a stronger result: the assumption of finite second moments for the recurrence times can now be dropped. (Received Octobr 30, 1956.)

\section{B. O. Koopman: Uniqueness conditions for a class of stochastic differential equations.}

The initial value problem is studied for the general stationary-transitional "birthdeath" process whose time differential equation is $P_{n}^{\prime}(t)=R_{n-1} P_{n-1}(t)-\left(R_{n}+L_{n}\right) P_{n}(t)$ $+L_{n+1} P_{n+1}(t)\left(P_{n}(t)\right.$ is the probability of state $n, P_{n}^{\prime}(t)$ its time derivative; the right and left transition probability coefficients $R_{n}$ and $L_{n}$ are perfectly general positive time-independent quantities; $n \geqq 0$ for the unilateral case; $n$ a general integer in the bilateral one.) Also, for its adjoint $Q_{n}^{\prime}(t)=L_{n} Q_{n-1}(t)-\left(L_{n}+R_{n}\right) Q_{n}(t)+R_{n} Q_{n+1}(t)$. In the unilateral case, it is proved that the necessary and sufficient condition for the uniqueness of the initial value problem is the divergence of the first of the two infinite triangular series $\sum_{n, m=1,0}^{\infty}\left(R_{n} R_{n+1} \cdots R_{n+m}\right) /\left(L_{n} L_{n+1} \cdots L_{n+m} L_{n+m+1}\right), \sum_{n, n=0}^{\infty}\left(L_{n+1}\right.$ $\left.\cdots L_{n+m}\right) /\left(R_{n} R_{n+1} \cdots R_{n+m}\right)$ while the adjoint will be unique if and only if the second series diverges. Equally simple necessary and sufficient conditions are given in the bilateral case; in addition to these series, two corresponding ones with indefinitely decreasing subscripts are used. The method applies analytic continued fraction theory to the Laplace transforms of these equations. (Work supported by the Office of Scientific Research of the U. S. Air Force.) (Received November 30, 1956.)

\section{S. C. Moy: Integral operators and Markov chain.}

Let $K$ be a linear operator on a Banach space into itself and $K^{*}$ be its adjoint. If $\left\|K^{n}\right\| \leqq c$ for all $n$ then the existence of a nonzero fixed point of $K$ implies the existence of a nonzero fixed point for $K^{*}$. Consider a transition function of a Markov chain as the adjoint of a linear operator on the space of bounded measurable functions, then the hypothesis of the theorem stated is satisfied. Therefore the following statement is concluded. There always exist absolute stationary probability distributions for any 
transition function of a Markov chain if finitely additive probability distributions are allowed. (Received November 13, 1956.)

320t. R. P. Pakshirajan: On the maximum partial sums of sequences of independent random variables.

Let $X_{\nu}, \nu=1,2, \cdots$ be a sequence of independent random variables with $E X_{\nu}=0, E X_{\nu}^{2}=\sigma_{\nu}^{2}$, and with distribution function $F_{\nu}(x)$. Set $S_{n}=X_{1}+X_{2}+\cdots$ $+X_{n}, S_{n}^{*}=\max _{1 \leqq \nu \leqq n}\left|S_{\nu}\right|$, and $s_{n}^{2}=\sum_{\nu=1}^{n} \sigma_{\nu}^{2}$. Assume $s_{n} \rightarrow \infty$ as $n \rightarrow \infty$. Erdös and Kac (Bull. Amer. Math. Soc. vol. 52 (1946) p. 292) found the limiting distribution of $S_{n}^{*}$ under the sole assumption that the $X_{\nu}$ satisfy the Central Limit Theorem. Chung (Trans. Amer. Math. Soc. vol. 64 (1948) pp. 205-233) found the rate of this convergence and proved some strong limit theorems for $S_{n}^{*}$ under assumptions stronger than existence of finite third moments for the $X_{\nu}$. In this paper we establish all of Chung's results under the condition $\left(\log s_{n}\right)^{-1 / 2} s_{n}^{-(2-23 \alpha)} \sum_{\nu=1}^{n} \int_{|x|>s_{n}}^{1-\alpha} \int x^{2} d F_{\nu}(x)=O(1)$. It is easily shown that Chung's conditions always imply ours. It may be observed that if our condition holds for some $\alpha=\alpha_{1}$, then it holds for all $\alpha \leqq \alpha_{1}$. If our condition holds for a maximum $\alpha$ in $3 / 4<\alpha<1$ then our conditions and Chung's conditions are equivalent. If our condition holds for a maximum $\alpha$ in $0<\alpha \leqq 3 / 4$, then it is much weaker than Chung's, as there exist random variables $X$, whose third absolute moments do not exist but which satisfy our condition. If the $X$, have a common distribution function our condition is satisfied if absolute moments of order $2+\delta(0<\delta<1)$ exist. Thus even in the identically distributed case our condition is much weaker than Chung's. The method of proof is the same as Chung's, the point of departure being the use of Berry's estimates for convergence to Normal involving only second moments in place of Essen's estimates involving third moments. (Received November 13, 1956.)

321t. M. P. Schützenberger: A generalization of the Fréchet-Cramér inequality to the case of Bayes estimation.

Let $f(x)$ be the a priori density function of $x ; g(y \mid x)$ the conditional density function of $y$. For fixed $x$, the set of $n$ independent $y$-variates is represented by $z$. The density function of $z$ is $f^{\prime}(z)$ and $g^{\prime}(x \mid z)$ is the a posteriori density function of $x$, for given $z$. The a posteriori variance of the Bayes estimate is $v_{z}^{2}=\int(x-\bar{x})^{2} g^{\prime}(x \mid z) d x$ and $v^{2}=E_{z} v_{z}^{2}=\int v_{x}^{2} f^{\prime}(z) d z$ is its average over $z . F=\int(\partial f(x) / \partial x)^{2}(f(x))^{-1} d x ; G=E_{x} G_{x}$ with $G_{x}=\int((\partial / \partial x) g(y \mid x))^{2}(g(y \mid x))^{-1} d y ; G^{\prime}=E_{z} G_{z}^{\prime}$ with $G_{z}^{\prime}=\int\left((\partial / \partial x) y^{\prime}(x \mid z)\right)^{2}(g(x \mid z))^{-1} d x$. The usual assumptions on $f$ and $g$, which insure that $F, G_{x}, G_{z}^{\prime}$ are finite are made. Since $O=F^{\prime}=\int\left((\partial / \partial x) f^{\prime}(z)\right)^{2}\left(f^{\prime}(z)\right)^{-1} d z$, it is easily seen that $F+n G=G^{\prime}$ (Third London Symposium on Information Theory, 1955, p. 18). Furthermore, it is a classical result that $v_{z}^{2} G_{z}^{\prime} \geqq 1$. Thus $v^{2}=E_{z} v_{z}^{2} \geqq\left(E_{z} 1 / v_{z}^{2}\right)^{-1} \geqq\left(E_{z} G_{z}^{\prime}\right)^{-1}=(F+n G)^{-1}$, which is the desired inequality that tends to the usual form when $n$ goes to infinity. It reduces to an equality if and only if $v^{2}=v_{z}^{2}=\left(G_{z}^{\prime}\right)^{-1}$ for all $z$, that is, if and only if $g^{\prime}(x \mid z)$ is gaussian with variance independent of $z$. If, furthermore, $y-x=t$ has a distribution $h(t)$ independent of $x$, this implies that $f(x)$ and $h(t)$ are also gaussian. (This work was supported in part by the Army (Signal Corps), the Air Force (Office of Scientific Research, Air Research and Development Command), and the Navy (Office of Naval Research).) (Received November 5, 1956.)

322. Esther Seiden: On a mathematical model for a problem of epidemiology.

The following problem was suggested by $D$. Blackwell: denote the number of susceptibles and infected individuals in a population by $s$ and $i$, respectively. Consider 
a Markovian stochastic model of the problem characterized by the following transitions within the time interval $(t, t+d t): 1$. Immigration $I \rightarrow I+1$ with probability $a d t+0(d t)$, 2. Immigration $S \rightarrow S+1$ with probability $b d t+0(d t)$, 3. Emigration $I \rightarrow I-1$ with probability $c I d t+0(d t), 4$. Emigration $S>S-1$ with probability $d S d t+0(d t)$, 5. Infection $I \rightarrow I+1, S \rightarrow S-1$ with probability $e I S d t+0(d t)$, where $a, b, c, d, e$ are non-negative constants. It is shown that in case the r.v. $I$ and $S$ are uncorrelated one can describe the population in terms of $E I$ and $E S$ as follows: The population will tend to a state of equilibrium independently of its initial state. The shape of the path will depend on the relative rates of passing from one state to another. If in addition it is assumed that $b$ is equal to $c$, then it is possible to describe the state of the population at any given time $t$. The system of differential equations for the deterministic model reduces to the above by replacing $E(I S)$ by $E I E S$. Hence in this case the conclusions drawn are in fact valid. (Received November 16,1956.)

\section{TOPOLOGY}

\section{R. D. Anderson: The group of all homeomorphisms of the uni-} versal curve.

Let $G$ be the group of all homeomorphisms of the universal curve $M$. It is known that $G$ may be given a metric as follows with respect to which $G$ is a complete metric space. For $g_{1}, g_{2} \in G, d\left(g_{1}, g_{2}\right)$ is the least upper bound of the set of numbers $\rho\left(g_{1}(x)\right.$, $\left.g_{2}(x)\right)+\rho\left(g_{1}^{-1}(x), g_{2}^{-1}(x)\right)$ for $x \in M$ and $\rho$ a distance function on $M$. It is known that $M$ is homogeneous. It is shown in this paper (by a fairly easy argument) that $G$ is zero-dimensional. (Received November 13, 1956.)

\section{B. J. Ball: On the normality of the product of two ordered spaces.}

First it is shown that for every nonparacompact ordered space $X$, there exists a compact ordered space $Y$ such that $X \times Y$ is not normal. Hence an ordered space is paracompact if and only if its product with every compact space is normal. Secondly, if $X$ is a nonparacompact ordered space in which every closed, bounded interval is compact, and $Y$ is a compact ordered space, then $X \times Y$ is normal if and only if no point of $Y$ has a left or right character equal to the character of either end-gap of $X$. This is then used to give a necessary and sufficient condition for the normality of $X \times Y$, where $X$ is a locally compact ordered space and $Y$ is a compact ordered space. (Received November 14, 1956.)

\section{R. H. Bing: Conditions under which monotone decompositions of $E^{3}$ are simply connected.}

An example is given of a monotone upper semicontinuous decomposition $G$ of $E^{3}$ such that the decomposition space is neither simply connected nor locally simply connected. The only nondegenerate element of $G$ is a solenoid. It is shown that if $H$ is a monotone upper semicontinuous decomposition of $E^{3}$, then the decomposition space is simply connected provided either each element of $H$ is locally connected or lies in a plane. If the decomposition space of $H$ is not simply connected, there is an element $h$ of $H$ such that the decomposition of $E^{3}$ whose only nondegenerate element is $h$ is not even locally simply connected. (Received November $13,1956$. )

326t. K. T. Chen: Integration of paths-a formal method for Lie groups.

The exponential homomorphism $\Theta$ with respect to the differentials $\pi_{1}, \cdots, \pi_{m}$ in a $C^{r}$ (or complex analytic) manifold $M$ of dimension $n$ is given by the formal power 
series $\Theta(\alpha)=1+\sum_{p=1}^{\infty} \sum \int_{\alpha} \pi_{i_{2}} \cdots \pi_{i_{p}} X_{i_{1}} \cdots X_{i_{p}}$ in the noncommutative indeterminates $X_{1}, \cdots, X_{m}$ for any path $\alpha:[a, b] \rightarrow M$. Denote by $\alpha[a, t]$ the portion of $\alpha$ with parameter running from $a$ to $t$. Both $\log \Theta(\alpha)$ and $(d / d t) \log \Theta(\alpha[a, t])$ are Lie elements which converge under the relations $\left[X_{i}, X_{l}\right]=\sum c_{i j k} X_{k}$ for $\alpha$ sufficiently short, $c_{i j k}$ 's being the structural constants of a Lie algebra. Thus one constructs the corresponding local Lie group and its Maurer-Cartan forms. The above results rely upon no differential equations. If $M$ is real with $r \geqq 2$ and $\pi_{1}, \cdots, \pi_{m}$, of rank $n$ over $M$, then any irreducible piecewise regular continuous path $\alpha$ is uniquely determined (up to change of parameter) by $\Theta(\alpha)$ and $\alpha(a)$. Let $\tilde{\theta}$ be the exponential homomorphism with respect to a base $\omega_{1}, \cdots, \omega_{m}$ of Maurer-Cartan forms of a real Lie group $G$, then any irreducible piecewise regular continuous path $\widetilde{\alpha}$ in $G$ is uniquely determined by $\tilde{\theta}(\alpha)$ up to left translation. Consequently $G$, if connected, is a homomorphic image of the group generated by all $\exp \sum \mu_{2} X_{i}, \mu_{i}$ 's running over real numbers. (Received October 15, 1956.)

\section{W. F. Davison: Topologies for function spaces of curves.}

Let $(X, T)$ be a curve space, with topology obtained from a mosaic of Peano spaces (see W. F. Davison, Convergent sequences and mosaics, Bull. Amer. Math. Soc. vol. 62 (1956) p. 180). On the set $X^{I}$ of all continuous functions from the unit interval into $(X, T)$ let $T_{c o}$ be the compact-open topology. Since $T_{C O}$-convergent sequences have unique limits the compact metric subspaces of $\left(X^{I}, T_{C O}\right)$ form a mosaic, which yields a mosaic topology $T_{M}$ on $X^{I}$. The Peano subspaces of $\left(X^{I}, T_{C O}\right)$ yield a curve topology $T_{C}$. The resulting inclusions $T_{C o} \subset T_{M} \subset T_{C}$ are in general proper. Every $T_{C}$-convergent sequence has a subsequence in a homotopic deformation of curves in $(X, T)$. Each topology $T_{C O}, T_{M}$, and $T_{C}$, relativized to the set $K$ of constant functions, yields a homeomorph of $(X, T) . K$ is closed for $T_{M}$ and $T_{C}$, but not necessarily for $T_{C o}$. The function $p: X^{I} \rightarrow X$ defined by $p(f)=f(0)$ is continuous and open for $T_{C O}, T_{M}$ and $T_{C}$; thus $(X, T)$ is a retract of $\left(X^{I}, T_{M}\right)$ and $\left(X^{I}, T_{C}\right)$. Since the set of functions $X^{I}$ satisfies the compatibility condition Fréchet equivalence $F$ is defined (see W. F. Davison, An equivalence relation for compact Hausdorff varieties, Bull. Amer. Math. Soc. vol. $61(1955)$ pp. 567). The quotient space $\left(X^{I} / F, T_{Q}\right)$ of $F$ and $\left(X^{I}, T_{C}\right)$ remains a curve space, with its topology described in terms of a mosaic of Peano spaces. (Received November 13, 1956.)

\section{8t. Robert Ellis: Locally compact transformation groups.}

Let $X$ be a locally compact Hausdorff space, let $T$ be a group of homeomorphisms of $X$, and let $\pi: X \times T \rightarrow X$ such that $\pi(x, t)=x t$ for $x \in X$ and $t \in T$. If $T$ is provided with a locally compact Hausdorff topology which makes $\pi$ unilaterally continuous and the maps $t \rightarrow s t$ and $t \rightarrow t s$ of $T$ into $T$ continuous for all $s \in T$, then $\pi$ is continuous. Using this result, it is shown that if $X$ is a locally compact Hausdorff space with a group structure in which multiplication is unilaterally continuous, then $X$ is a topological group. (Received November 13, 1956.)

\section{Leonard Gillman: Real-compact spaces (Q-spaces).}

It is proposed that Hewitt's $Q$-spaces be termed real-compact. (For background, see Proc. Amer. Math. Soc. vol. 5 (1954) pp. 447-455.) Let $X$ be a completely regular space. Hewitt proved that a maximal ideal $M$ in $C(X)$ is real $(C / M$ is the real field) iff its family of zero-sets has the countable intersection property (c.i.p.). Call an ultrafilter $\mathcal{U}$ on $X$ real if the family of all zero-sets in $\mathcal{U}$ has the c.i.p. The following results, among others, prompt the proposal. I. $X$ is compact iff every maximal ideal in $C$ is 
fixed; $X$ is real-compact iff every real maximal ideal in $C$ is fixed. II. $X$ is compact iff every ultrafilter converges; $X$ is real-compact iff every real ultrafilter converges. III. Any product of compact spaces is compact (Bourbaki proof uses convergence of ultrafilters); any product of real-compact spaces is real-compact (analogous proof uses convergence of real ultrafilters). IV. In any structure, $X$ is precompact iff every ultrafilter is a Cauchy filter; $X$ is pre-real-compact (its completion is real-compact) iff every real ultrafilter is a Cauchy filter. (Necessity in this last was proved by Shirota; sufficiency is an unpublished result of Isbell.) (Received November 13, 1956.)

330t. A. J. Goldman: A Cech theory of fundamental groups and covering spaces.

Let $X$ be a connected (but not necessarily locally connected) space. By $F(X)$ we mean the inverse system of fundamental groups of nerves of $X$ 's open coverings, together with the natural homomorphisms between these groups. I. The inverse limit $\pi^{\prime}(X)$ of $F(X)$ obeys the homotopy axiom, obeys the continuity axiom on compact spaces, and is independent of the basepoint if $X$ is pathwise connected. If $X$ is also locally pathwise connected and semilocally-1-connected, then $\pi^{\prime}(X)$ is naturally isomorphic to the ordinary fundamental group $\pi(x)$; it follows that the 1-dimensional Cech and singular homology groups of such an $X$ coincide. II. Using $F(X)$, procedures are given for classifying those covering spaces (both connected and disconnected) over $X$ which have "simplicial approximations." Every finite-sheeted covering space is shown to be of this type. The classification is carried out in detail for a " $\sin (1 / x)$ " space and for the dyadic solenoid. (Received October 24, 1956.)

331. M. E. Hamstrom (p) and Eldon Dyer: The space of homeomorphisms on a two-manifold.

It is known that the space $H\left(S^{2}\right)$ of homeomorphisms of a 2-sphere onto itself is locally arc-wise connected [M. K. Fort, Proc. Amer. Math. Soc. vol. 1 (1950) pp. 5962]. Using a generalization of a technique of J. H. Roberts [Summary of Lectures and Seminars, Summer Institute in Set Theoretic Topology, Madison, Wis. 1955, p. 100] it is shown that the space $H(C)$ of homeomorphisms of a compact 2-manifold with boundary $B$ (possibly null) onto itself which leave $B$ fixed has the following property: if $\epsilon>0$, there is a $\delta>0$ such that if $K$ is a compactum, $\operatorname{dim} K<\infty$, and $f: K \rightarrow H(C)$ has diameter less than $\delta$, then $f$ can be extended to a map on the cone of $K$ having diameter less than $\epsilon$. Using a selection theorem of E. Michael [Ann. of Math., to appear], it is shown that if $p: X \rightarrow B$ is a 0 -regular map, $B$ is a compactum, $\operatorname{dim} B<\infty$, and for each $b \in B, f^{-1}(b)$ is a compact 2 -manifold, then $(X, p, B)$ is a locally trivial fibre space. It is also shown that if $K$ is as before, $P^{3}$ is the space of rotations of the 2-sphere, and $f: K \rightarrow J$, the identity component of $H\left(S^{2}\right)$, then there is a map $F: K \times I \rightarrow J$ such that $F \mid K \times(0)=f, F(K \times(1)) \subset P^{3}$, and if $f(x) \in P^{3}$, then $F(x, t)$ $=f(x)$ for all $t \in I$. This shows that $J$ is of the same weak homotopy type as $P^{3}$, the real projective 3-space. Certain of these results are similar to results of J. Cerf (unpublished). The authors are not aware of Cerf's arguments. (Part of this work was sponsored by the National Science Foundation.) (Received November 13, 1956.)

332. Melvin Henriksen ( $\mathrm{p}$ ) and J. R. Isbell: On the stone-Cech compactification of a product of two spaces.

As usual, $\beta X$ denotes the Stone-Cech compactification of the completely regular (c.r.) space $X$ (E. Čech, Ann. of Math. vol. 38 (1937) pp. 823-844). If $X$ is dense in 
a compact space $B X$ and there is a homeomorphism of $\beta X$ upon $B X$ keeping $X$ pointwise fixed, we write $\beta X=B X$. A c.r. space $X$ is pseudo-compact if every continuous real-valued function on $X$ is bounded (E. Hewitt, Trans. Amer. Math. Soc. vol. 64 (1948) pp. 45-99). Theorem 1. If $X$ and $Y$ are infinite c.r. spaces such that $\beta(X \times Y)$ $=\beta X \times \beta Y$, then $X \times Y$ is pseudo-compact. Theorem 2 . If $X$ is compact and satisfies the first axiom of countability, and if $Y$ is pseudo-compact, then $\beta(X \times Y)=\beta X \times \beta Y$. Theorem 3. If $X$ is compact, and if $Y$ is pseudo-compact and has a countable dense subset, then $\beta(X \times Y)=\beta X \times \beta Y$. (Received November 6, 1956.)

\section{Melvin Henriksen and Meyer Jerison (p): A nonnormal sub- space of $\beta N$.}

I. Theorem. Every nonnormal space contains a closed subset which is not a zeroset $\left(=f^{-1}(0)\right.$ for some continuous $\left.f\right)$. Proof. Disjoint zero-sets can always be separated by disjoint open sets (Hewitt, Trans. Amer. Math. Soc. vol. 64 (1948) p. 61). II. Let $\left\{N_{\alpha}\right\}$ be $c$ infinite subsets of a countable set $N$, such that $N_{\alpha} \cap N_{\gamma}$ is finite for $\alpha \neq \gamma$. In $\beta N$, the Stone-Cech compactification of $N$ (discrete), let $Y_{\alpha}=N_{\alpha}-N$, and $Y$ consist of one point from each $Y_{\alpha}$. Then $Y$ has $c$ points, is discrete in $\beta N$, and is closed in $X=N \cup Y . X$ is nonnormal-the following proof also works for other examples: not every function on the closed set $Y$ can be extended continuously to $X$ because there are $2^{c}$ functions on $Y$ but only $c$ (on $N$, hence) on $X$. Every subset of $X$ is a $G_{\delta}$-set; by Theorem I, $X$ contains a closed $G_{\delta}$ which is not a zero-set. III. (Known) $\beta N-N$ is not extremally disconnected. Proof. If $A_{1}, A_{2}$ are disjoint sets in $X$ which cannot be separated by open sets, then $U\left\{Y_{\alpha}: Y_{\alpha} \cap A_{i} \neq \phi\right\}, i=1,2$, are disjoint open sets in $\beta N-N$ whose closures intersect. (Received November $13,1956$.

\section{J. G. Hocking: Extensions of a monotone mapping on a 2- sphere.}

A recent result of Floyd and Fort characterizes a monotone mapping of a 2-sphere onto itself in terms of an extension of the mapping to a homeomorphism on the bounded complement of the 2-sphere. [Proc. Amer. Math. Society vol. 4 (1953) pp. 828-830]. Similar extensions are possible for the general monotone mapping of $S^{2}$ as the following result shows: Theorem: Let $f$ be a monotone mapping of $S^{2}$ onto the cactoid $K$. Then there exists an extension $g$ of $f$ mapping the solid sphere onto the solid cactoid such that on each open 3-cell in the solid cactoid, $g^{-1}$ is a homeomorphism. As a corollary to the method of proof, one easily establishes the following result, too: Theorem: Let $f: S^{2}>K$ be monotone. Then $f$ may be approximated arbitrarily closely by a mapping $g$ such that on each open 2-cell in $K$ not containing a cut-point of $K$, $g^{-1}$ is a homeomorphism. The key lemma in the argument is the factorization $f=m_{0} m$ of a monotone mapping $f$ where $m_{0}$ is $n$-monotone for each $n$. (Received November 13 , 1956.)

\section{J. G. Horne: o-ideals in semirings and a universal compactifica- tion procedure.}

A semi-ring is a set $R$ which is a semigroup with respect to two operations " + " and " ." and $a(b+c)=a b+a c$. For simplicity, assume both semigroups commutative. Sufficient conditions are placed on a transitive relation $\gg$ on $R$ in order that the space of maximal 》-ideals form a compact Hausdorff space in the dual Stone topology, ( $A \gg$-ideal is a $\gg$-directed ideal; when $f \gg e$ means $f e=f$, they are $o$-ideals.) Consequences include: (1) a generalization of a theorem of Shirota concerning $R$-lattices (Osaka Math. J. vol. 4 (1952)); (2) a natural sufficient condition on a commutative 
ring $R$ with identity in order that its space of maximal $o$-ideals be compact (viz., if $f \gg e$ means there exists $e^{\prime}$ such that $f e^{\prime}=f$ and $e^{\prime} e=e^{\prime}$ then $\gg$ is a dense order on $R$ ); (3) every compact extension of a completely regular space can be obtained as the maximal 》-ideal space of some family $S$ of its open sets and some relation $\gg$ on $S$; (4) an "internal" construction of a compact extension of any uniform space; (5) a result of Fan and Gottesman concerning a compactification of Freudenthal and Wallman (Indagationes Math. vol. 14 (1952)). (Received November 13, 1956.)

336. J. R. Isbell: Weak uniformities induced by families of realvalues functions.

A large covering of uniform space has a uniformly continuous partition of unity subordinated to it if the covering is star-bounded, i.e. for some natural number $n$, no element of the covering meets more than $n$ other elements of the covering. (The term and concept are due to G. D. Mostow, who will publish some Euclidean results which may help on the present problem.) This means that the covering can be realized by a mapping into a polyhedron. Now if a uniformity is the weak uniformity induced by a family of real-valued functions, then it has a basis consisting of countable starbounded coverings. The converse is not true, because the relevant polyhedra cannot always be embedded in a Euclidean space by a uniform equivalence. (The metric Lobachewski plane is a counterexample, pointed out by J. E. Nelson.) However, the weak uniformities induced by certain kinds of families of functions, which are algebras generalizing the algebras of all continuous functions, are characterized in this paper. The key lemma is a very weak result on embeddings of polyhedra. The general embedding theorem of dimension theory is used to get a homeomorphism into $(2 n+1)$ space, and the mapping is then modified in $(2 n+2)$-space. (Received November 13, 1956.)

\section{M. A. Kervaire: Normal bundle to a sphere in euclidean space.}

Define two regular immersions of $S_{d}$ into euclidean $(d+n)$-space as equivalent if they induce equivalent normal bundles over $S_{d}$. Equivalence classes of immersions of $S_{d}$ into $E_{d+n}$ form a group $J_{d, n}$ under the operation of joining the images by a tube. It is shown that $J_{d, n}=0$ for $n \geqq d+1$ and $J_{d, d} \cong Z, Z_{2}$ or 0 according to $d$ is even, odd and $S_{d}$ not parallelizable, or $S_{d}$ parallelizable respectively. Similar groups $P_{d, n}$ may be defined using imbeddings of $S_{d}$ into $E_{d+n}$. One has $P_{d, d}=0$ for every $d$. It is not known, whether $P_{d, n} \neq 0$ for some pair $(d, n)$. (Received November $\left.13,1956.\right)$

\section{G. R. Livesay: Concerning real valued maps of $S_{n}$.}

The following theorem is proved: Let $0 \leqq d \leqq$ diam. $S_{n}, f: S_{n} \rightarrow E_{1}$ continuous, $\epsilon>0$. Then in a sufficiently fine triangulation in which the antipodal map, $T$, is simplicial, there is a representative, $\gamma_{n-1}$, of the non zero class of $H_{n-1}\left(S_{n}, T: Z_{2}\right)$ such that for $x \in\left|\gamma_{n-1}\right|$, there exists $y \in S_{n}$ with $\rho(x, y)=d,|f(x)-f(y)|<\epsilon$. ( $\rho$ is the metric is $S_{n}$.) The only notion used which may be new is a definition for the width of a curve: Let $\alpha: S_{1} \rightarrow X$ (metric) be continuous, $H$ the space of maps of degree 1 of $S_{1} \rightarrow S_{1}$. Then define $w(\alpha)=\lim \inf _{h \in H} \max _{0 \leqq \theta \leqq} \delta(\alpha h(\theta), \alpha h(-\theta))$, where $\delta$ is the metric in $X$. This width agrees with the usual definition for convex curves in the plane. (A part of this work was done under contract no. 7174 with the Office of Naval Research.) (Received November 13, 1956.)

\section{D. M. Mesner: Linear graphs with a condition on 2-chains.}

In a nonoriented linear graph with $n$ points and $L$ lines let $s$ denote the largest integer such that there is a complete subgraph with $s$ points. Graphs are considered 
which satisfy (1): two points not joined by a line are joined by at most $D$ chains of two lines. There are known inequalities in $n, L$ and $s$ which say that if $L$ is large, then $s$ must be fairly large, loosely speaking. It is shown here that if (1) holds, $s$ must be considerably larger, still speaking loosely. More specifically, and defining $Z=n(n-1)$ $-2 L, Z \geqq \max \left[2(n-s)(s-D), 2^{-1}(n+s-2 D), 2^{-1}(n-D)(n-s), n^{2} / s-n\right]$. The proof makes use of incidence matrices, in which $Z$ is the number of off-diagonal 0 's and (1) becomes a condition on the inner products of certain pairs of rows taken as vectors. Conditions on 2-chains arise naturally in graphs obtained by identifying points with treatments in a PBIB experimental design and joining pairs of points which correspond to first associates. (Received November 14, 1956.)

\section{C. J. Neugebauer: B-sets and fine cyclic elements.}

An $A$-set of a Peano space $P$ can be defined as a nondegenerate closed subset $A$ of $P$ such that either $A=P$ or else every component of $P-A$ has a single frontier point. A proper cyclic element of $P$ can be defined as a cyclic $A$-set of $P$. An immediate generalization of the above concepts is the following. A $B$-set $B$ of a Peano space $P$ is a nondegenerate continuum of $P$ such that either $B=P$ or else every component of $P-B$ decomposes into a finite number of points. A fine cyclic element (introduced first in a slightly different context by L. Cesari, Bull. Amer. Math. Soc. Abstract 61-6-698) of $P$ is then defined to be a $B$-set which remains connected after removing any finite set of points. In order to obtain a theory of $B$-sets and fine cyclic elements as complete as possible as the theory of $A$-sets and proper cyclic elements one is forced to restrict $P$ to be of finite degree of multicoherence. The paper shows that the properties of $B$ sets and fine cyclic elements of such Peano spaces are suitable extensions of the corresponding properties of $A$-sets and proper cyclic elements. (Received November 13, 1956).

\section{C. D. Papakyriakopoulos: $A$ proof of Dehn's lemma.}

The following theorem known as Dehn's lemma [Math. Ann. vol. 69 (1910) p. 147] is proved: Let $M$ be a 3-manifold, compact or not, with boundary which may be empty, and in $M$ let $D$ be a 2-cell with self-intersections (singularities), having as boundary the simple closed polygonal curve $C$ and such that, there exists a closed neighborhood of $C$ in $D$ which is an annulus (i.e. no point of $C$ is singular). Then there exists a 2-cell $D_{0}$ with boundary $C$, semi-linearly imbedded in $M$. A consequence of Dehn's lemma is that, a knot $K$ in the 3-sphere $S^{3}$ is unknotted if and only if $\pi_{1}\left(S^{3}-K\right)$ is free cyclic [Math. Ann. vol. 69 (1910) p. 158, Satz 2]. The theorem is proved in the case where $M$ is orientable. From this and a theorem of I. Johansson [Math. Ann. vol. 115 (1938) p. 695 ] follows that, the theorem holds also for any nonorientable $M$. (Received October $31,1956$.

\section{2t. C. D. Papakyriakopoulos: The sphere theorem.}

The following theorem is proved: Let $M$ be an orientable 3-manifold, compact or not, with boundary which may be empty, such that $\pi_{z}(M) \neq 0$, and which can be topologically imbedded in a 3-manifold $N$, having the following property: The first homology group of any nontrivial (but not necessarily proper) subgroup of $\pi_{1}(N)$, has an element of infinite order (n.b. in particular this holds if $\left.\pi_{1}(N)=1\right)$. Then there exists a 2-sphere $S$ semi-linearly imbedded in $M$, such that $S$ is not homotopic to 0 in $M$. The proof of this theorem is analogous to the author's proof of Dehn's lemma [Bull. Amer. Math. Soc. Abstract 63-2-341]. (Received October 31, 1956.) 
343t. C. D. Papakyriakopoulos: $A$ proof of the asphericity of knots.

The following theorem is proved: Let $U$ be a nonempty proper open connected subset of the 3-sphere $S^{3}$. Then $U$ is aspherical if and only if $S^{3}-U$ belongs to one and the same component of $S^{3}-S^{2}$, for any 2-sphere $S^{2}$ semi-linearly imbedded in $U$. This solves a problem of J. H. C. Whitehead [Fund. Math. vol. 32 (1939) p. 161], and its proof is based on the theorem contained in the author's abstract. The sphere theorem [Bull. Amer. Math. Soc. Abstract 63-2-342]. A corollary of the above theorem is the following: If $F$ is a nonempty proper closed connected subset of $S^{3}$, then each component of $S^{3}-F$ is aspherical. From this follows immediately: If $F$ is a connected graph or knot, then $S^{\mathbf{3}}-F$ is aspherical. (Received October 31, 1956.)

\section{4t. C. D. Papakyriakopoulos: $A$ proof of Hopf's conjecture.}

The following theorem known as Hopf's conjecture is proved: If $U$ is an open connected subset of the 3-sphere, then $\pi_{1}(U)$ has no element of finite order. The proof is based on the theorem contained in the author's abstract. The sphere theorem [Bull. Amer. Math. Soc. Abstract 63-2-342], and the following simple consequence of a theorem due to P. A. Smith: The fundamental group of an aspherical polyhedron (of finite dimension) has no element of finite order [Hurewicz, Proc. Acad. Amsterdam vol. 39 (1936) p. 216, 1. 31]. (Received October 31, 1956.)

345. J. E. L. Peck (p) and A. L. Dulmage: Games on a compact set.

The fundamental theorem of the theory of games has been extended by Kneser (C. R. Acad. Sci. Paris vol. 234 (1952) pp. 2418-2420) and Nikaidô (J. Math. Soc. Japan vol. 5 (1953) pp. 86-94). We give an extension which goes beyond both of these. The theorem is as follows: $\widetilde{X}$ is a convex subset of a real linear space, $Y$ is any subset of a real linear space and $\widetilde{Y}$ is the convex set generated by $Y$. A real function $f$ is defined on $\widetilde{X} \times \widetilde{Y}$ and $f(x, y)$ is a concave function of $x \in \widetilde{X}$ and a convex function of $y \in \widetilde{Y}$. If $\widetilde{X}$ is compact in a topology which is such that for every $y \in Y, f(x, y)$ is an upper semi-continuous function of $x \in \widetilde{X}$, then $\sup _{x \in \widetilde{X}} \inf _{y \in Y} f(x, y)$ $\geqq \inf _{y \in \widetilde{Y}} \sup _{x \in \widetilde{X}} f(x, y)$. As a corollary one obtains that if in a game, one of the pure strategy spaces $X$ is a compact and Hausdorff, while the pay-off function is such that if $y$ is any pure strategy for the other player, $f(x, y)$ is a continuous function of $x$, then the game has a value. Some pertinent examples are given. (Received November 7, 1956.)

346. R. W. Rector: $A$ system of linear equations for the seven ring.

Birkhoff and Lewis (Trans. Amer. Math. Soc. vol. 60 (1946) pp. 355-451) conclude their attack on the Four Color Problem with a partial analysis of the $n$-ring with particular attention to the 6-ring and the 7-ring. The present author has previously indicated in a preliminary report (Bull. Amer. Math. Soc. vol. 62 (1956) p. 399) an extension of the list of linear relations for the 7-ring and shown the existence of 126 linearly independent relations for the 7-ring. Utilizing these results a system of 36 linear equations can be obtained; the solution of this system would yield formulas expressing the constrained polynomials of the 7-ring in terms of free chromatic polynomials of maps containing fewer regions than the original map. (Received November 13,1956.)

347. P. E. Thomas: The Pontrjagin cohomology operations and rings with divided powers. Preliminary Report.

Following H. Cartan (see Séminaire H. Cartan, E.N.S. 1954-1955, Chapter 7) we define a ring with divided powers to be a commutative, graded ring $A=\sum A_{k}$ (de- 
gree $\left.A_{k}=2 k\right)$, together with a set of functions $\gamma_{r}(r=0,1 \cdots)$ such that: (1) $\gamma_{r}$ : $A_{k} \rightarrow A_{k r}$, (2) $\gamma_{0}(x)=$ unit of $A, \gamma_{1}(x)=x$, (3) $\gamma_{r}(x) \gamma_{s}(x)=\left(\gamma_{1} s\right) \gamma_{r+s}(x),(4) \gamma_{t}(x+y)$ $=\sum \gamma_{r}(x) \gamma_{s}(y)$, (5) $\gamma_{r}(x y)=r ! \gamma_{r}(x) \gamma_{r}(y)$ for $r \geqq 2$, (6) $\gamma_{r}\left(\gamma_{s}(x)\right)=\epsilon_{r, s} \gamma_{r s}(x)$. Here $(r, s)=(r+s) ! /(r ! s !)$ and $\epsilon_{r, s}=(s, s-1)(2 s, s-1) \cdots((r-1) s, s-1)$. The concept also clearly extends to bi-graded rings. A ring with divided powers will be called $p$-cyclic if each summand $A_{k}$ is a cyclic group of infinite order or order a power of a prime (after Eilenberg-MacLane). Let $K$ be a complex, and let $A$ be a ring with divided powers. Define the cohomology ring of $K$ with coefficients in $A, H^{*}(K ; A)$, as the bi-graded ring $\sum_{i, j} H^{i}\left(K ; A_{j}\right)$. Restricting the ring $A$ to be $p$-cyclic, we have THEOREM: $H^{*}(K ; A)$ is a ring with divided powers. The functions $\gamma_{r}(r=0,1 \cdots)$ are given by the Pontrajagin cohomologyoperations $\mathfrak{B}_{r}: H^{2 n}\left(K ; A_{k}\right) \rightarrow H^{2 r n}\left(K ; A_{r k}\right)$. The Pontrjagin operations are defined in Proc. Nat. Acad. Sci. vol. 42 (1956) pp. 266-269; however, the definition must be changed slightly in order to use the ring $A$ as coefficients. (Received November 14, 1956.)

\title{
348. R. F. Williams: Local contractions of continuous curves.
}

The purpose of this paper is to find an additional, purely topological, hypothesis for compact metric spaces $X$, such that the following theorem holds: if $f$ is a local contraction of $X$ onto itself, then $f$ is a local isometry. For definitions, counter-examples, and reference, see an earlier paper, (Proc. Amer. Math. Soc. vol. 5 (1954) p. 652). That $X$ be a dendrite (acyclic continuous curve) with only countably many end-points is shown to suffice. It is shown to be insufficient to assume any of the following: $X$ is a simple closed curve; $X$ is a dendrite, even in the plane; $X$ is a (topological) sphere of any dimension; or $X$ is a (topological) $n$-cell, for $n \geqq 2$. (Received November 13,1956 .)

\author{
R. D. Schafer, \\ Associate Secretary
}

